\title{
Linear superposition in nonlinear wave dynamics
}

\author{
A. Babin and A. Figotin \\ Department of Mathematics, University of California at Irvine, CA 92697
}

September 27, 2018

\begin{abstract}
We study nonlinear dispersive wave systems described by hyperbolic PDE's in $\mathbb{R}^{d}$ and difference equations on the lattice $\mathbb{Z}^{d}$. The systems involve two small parameters: one is the ratio of the slow and the fast time scales, and another one is the ratio of the small and the large space scales. We show that a wide class of such systems, including nonlinear Schrodinger and Maxwell equations, Fermi-Pasta-Ulam model and many other not completely integrable systems, satisfy a superposition principle. The principle essentially states that if a nonlinear evolution of a wave starts initially as a sum of generic wavepackets (defined as almost monochromatic waves), then this wave with a high accuracy remains a sum of separate wavepacket waves undergoing independent nonlinear evolution. The time intervals for which the evolution is considered are long enough to observe fully developed nonlinear phenomena for involved wavepackets. In particular, our approach provides a simple justification for numerically observed effect of almost non-interaction of solitons passing through each other without any recourse to the complete integrability. Our analysis does not rely on any ansatz or common asymptotic expansions with respect to the two small parameters but it uses rather explicit and constructive representation for solutions as functions of the initial data in the form of functional analytic series.
\end{abstract}

\section{Introduction}

The principal object of our studies here is a general nonlinear evolutionary system which describes wave propagation in homogeneous media governed either by a hyperbolic PDE's in $\mathbb{R}^{d}$ or by a difference equation on the lattice $\mathbb{Z}^{d}, d=1,2,3, \ldots$ is the space dimension. We assume the evolution to be governed by the following equation with constant coefficients

$$
\partial_{\tau} \mathbf{U}=-\frac{\mathrm{i}}{\varrho} \mathbf{L}(-\mathrm{i} \nabla) \mathbf{U}+\mathbf{F}(\mathbf{U}),\left.\mathbf{U}(\mathbf{r}, \tau)\right|_{\tau=0}=\mathbf{h}(\mathbf{r}), \mathbf{r} \in \mathbb{R}^{d},
$$

where (i) $\mathbf{U}=\mathbf{U}(\mathbf{r}, \tau), \mathbf{r} \in \mathbb{R}^{d}, \mathbf{U} \in \mathbb{C}^{2 J}$ is a $2 J$ dimensional vector; (ii) $\mathbf{L}(-\mathrm{i} \nabla)$ is a linear self-adjoint differential (pseudodifferential) operator with constant coefficients with 
the symbol $\mathbf{L}(\mathbf{k})$, which is a Hermitian $2 J \times 2 J$ matrix; (iii) $\mathbf{F}$ is a general polynomial nonlinearity; (iv) $\varrho>0$ is a small parameter. The form of the equation suggests that the processes described by it involve two time scales. Since the nonlinearity $\mathbf{F}(\mathbf{U})$ is of order one, nonlinear effects occur at times $\tau$ of order one, whereas the natural time scale of linear effects, governed by the operator $\mathbf{L}$ with the coefficient $1 / \varrho$, is of order $\varrho$. Consequently, the small parameter $\varrho$ measures the ratio of the slow (nonlinear effects) time scale and the fast (linear effects) time scale. A typical example an equation of the form (1.1) is nonlinear Schrodinger equation (NLS) or a system of NLS. Another one is the Maxwell equation in a periodic medium when truncated to a finite number of bands, and more examples are discussed below.

We assume further that the initial data $\mathbf{h}$ for the evolution equation (1.1) to be the sum of a finite number of wavepackets $\mathbf{h}_{l}, l=1, \ldots, N$, i.e.

$$
\mathbf{h}=\mathbf{h}_{1}+\ldots+\mathbf{h}_{N}
$$

where the monochromaticity of every wavepacket $\mathbf{h}_{l}$ is characterized by another small parameter $\beta$.

The well known superposition principle is a fundamental property of every linear evolutionary system, stating that the solution $\mathbf{U}$ corresponding to the initial data $\mathbf{h}$ as in (1.2) equals

$$
\mathbf{U}=\mathbf{U}_{1}+\ldots+\mathbf{U}_{N}, \text { for } \mathbf{h}=\mathbf{h}_{1}+\ldots+\mathbf{h}_{N},
$$

where $\mathbf{U}_{l}$ is the solution to the same linear problem with the initial data $\mathbf{h}_{l}$.

Evidently the standard superposition principle can not hold exactly as a general principle in the presence of a nonlinearity, and, at the first glance, there is no expectation for it to hold even approximately. We have discovered though that the superposition principle does hold with a high accuracy for general dispersive nonlinear wave systems provided that the initial data are a sum of generic wavepackets, and this constitutes the subject of this paper. Namely, the superposition principle for nonlinear wave systems states that the solution $\mathbf{U}$ corresponding to the multi-wavepacket initial data $\mathbf{h}$ as in (1.2) equals

$$
\mathbf{U}=\mathbf{U}_{1}+\ldots+\mathbf{U}_{N}+\mathbf{D} \text {, for } \mathbf{h}=\mathbf{h}_{1}+\ldots+\mathbf{h}_{N} \text {, where } \mathbf{D} \text { is small. }
$$

As to the particular form (1.1) we chose to be our primary one, we would like to point out that many important classes of problems involving small parameters can be readily reduced to the framework of (1.1) by a simple rescaling. It can be seen from the following examples. First example is a system with a small factor before the nonlinearity

$$
\partial_{t} \mathbf{v}=-\mathrm{i} \mathbf{L} \mathbf{v}+\alpha \mathbf{f}(\mathbf{v}),\left.\mathbf{v}\right|_{t=0}=\mathbf{h}, 0<\alpha \ll 1
$$

where initial data are bounded uniformly in $\alpha$. Such problems are reduced to (1.1) by the time rescaling $\tau=t \alpha$. Note that now $\varrho=\alpha$ and the finite time interval $0 \leq \tau \leq \tau_{*}$ corresponds to the long time interval $0 \leq t \leq \tau_{*} / \alpha$. 
Second example is a system with small initial data on a long time interval. The system here is given and has no small parameters but the initial data are small, namely

$$
\begin{gathered}
\partial_{t} \mathbf{v}=-\mathrm{i} \mathbf{L} \mathbf{v}+\mathbf{f}_{0}(\mathbf{v}),\left.\mathbf{v}\right|_{t=0}=\alpha_{0} \mathbf{h}, 0<\alpha_{0} \ll 1, \text { where } \\
\mathbf{f}_{0}(\mathbf{v})=\mathbf{f}_{0}^{(m)}(\mathbf{v})+\mathbf{f}_{0}^{(m+1)}(\mathbf{v})+\ldots,
\end{gathered}
$$

where $\alpha_{0}$ is a small parameter and $\mathbf{f}^{(m)}(\mathbf{v})$ is a homogeneous polynomial of degree $m \geq 2$. After the rescaling $\mathbf{v}=\alpha_{0} \mathbf{V}$ we obtain the following equation with a small nonlinearity

$$
\partial_{t} \mathbf{V}=-\mathrm{i} \mathbf{L V}+\alpha_{0}^{m-1}\left[\mathbf{f}_{0}^{(m)}(\mathbf{V})+\alpha_{0} \mathbf{f}^{0(m+1)}(\mathbf{V})+\ldots\right],\left.\quad \mathbf{V}\right|_{t=0}=\mathbf{h},
$$

which is of the form of (1.4) with $\alpha=\alpha_{0}^{m-1}$. Introducing the slow time variable $\tau=t \alpha_{0}^{m-1}$ we get from the above an equation of the form (1.1), namely

$$
\partial_{\tau} \mathbf{V}=-\frac{\mathrm{i}}{\alpha_{0}^{m-1}} \mathbf{L V}+\left[\mathbf{f}^{(m)}(\mathbf{V})+\alpha_{0} \mathbf{f}^{(m+1)}(\mathbf{V})+\ldots\right],\left.\quad \mathbf{V}\right|_{t=0}=\mathbf{h}
$$

where the nonlinearity does not vanish as $\alpha_{0} \rightarrow 0$. In this case $\varrho=\alpha_{0}^{m-1}$ and the finite time interval $0 \leq \tau \leq \tau_{*}$ corresponds to the long time interval $0 \leq t \leq \frac{\tau_{*}}{\alpha_{0}^{m-1}}$ with small $\alpha_{0} \ll 1$.

Very often in theoretical studies of equations of the form (1.1) or ones reducible to it a functional dependence between $\varrho$ and $\beta$ is imposed, resulting in a single small parameter. The most common scaling is $\varrho=\beta^{2}$. The nonlinear evolution of wavepackets for a variety of equations which can be reduced to the form (1.1) was studied in numerous physical and mathematical papers, mostly by asymptotic expansions of solutions with respect to a single small parameter similar to $\beta$, see [11, [14], 18, [20, 23, 28, 29], 34], 38, 39, 40] and references therein. Often the asymptotic expansions are based on a specific ansatz prescribing a certain form to the solution. In our studies here we do not use asymptotic expansions with respect to a small parameter and do not prescribe a specific form to the solution, but we impose conditions on the initial data requiring it to be a wavepacket or a linear combination of wavepackets. Since we want to establish a general property of a wide class of systems, we apply a general enough dynamical approach. There is a number of general approaches developed for the studies of high-dimensional and infinite-dimensional nonlinear evolutionary systems of hyperbolic type, [10], 13], 19], 22, [27], [31, [35, [39, 41], 43], [45]) and references therein. We develop here an approach which allows to exploit specific properties of a certain class of initial data, namely wavepackets and their linear combinaions, which comply with the symmetries of equations. Such a class of the initial data is obviously lesser than all possible initial data. One of the key mathematical tools developed here for the nonlinear studies is a refined implicit function theorem (Theorem 4.25). This theorem provides a constructive and rather explicit representation of the solution to an abstract nonlinear equation in a Banach space as a certain functional series. The representation is explicit enough to prove the superposition principle and is general enough to carry out the studies of the problem without imposing restrictions on dimension of the problem, structural 
restrictions on nonlinearities or a functional dependence between the two small parameters $\varrho, \beta$.

As we have already stated the superposition principle holds with high accuracy for linear combinations of wavepackets. A wavepacket $\mathbf{h}(\beta, \mathbf{r})$ can be most easily described in terms of its Fourier transform $\tilde{\mathbf{h}}(\beta, \mathbf{k})$. Simply speaking, wavepacket $\tilde{\mathbf{h}}(\beta, \mathbf{k})$ is a function which is localized in $\beta$-neighborhood of a given wavevector $\mathbf{k}_{*}$ (the wavepacket center) and as a vector is an eigenfunction of the matrix $\mathbf{L}(\mathbf{k})$, details of the definition of the wavepacket can be found in the following Section 2. The simplest example of a wavepacket is a function of the form

$$
\tilde{\mathbf{h}}(\beta, \mathbf{k})=\beta^{-d} \hat{h}\left(\frac{\mathbf{k}-\mathbf{k}_{*}}{\beta}\right) \mathbf{g}_{n}\left(\mathbf{k}_{*}\right), \mathbf{k} \in \mathbb{R}^{d},
$$

where $\mathbf{g}_{n}\left(\mathbf{k}_{*}\right)$ is an eigenvector of the matrix $\mathbf{L}\left(\mathbf{k}_{*}\right)$ and $\hat{h}(\mathbf{k})$ is a Schwartz function (i.e. it is infinitely smooth and rapidly decaying one). Note that the inverse Fourier transform $\mathbf{h}(\beta, \mathbf{r})$ of $\tilde{\mathbf{h}}(\beta, \mathbf{k})$ has the form

$$
\mathbf{h}(\beta, \mathbf{r})=h(\beta \mathbf{r}) \mathrm{e}^{\mathrm{i} \mathbf{k}_{*} \mathbf{r}} \mathbf{g}_{n}\left(\mathbf{k}_{*}\right), \mathbf{r} \in \mathbb{R}^{d}
$$

where $h(\mathbf{r})$ is a Schwartz function, and obviously has a large spatial extension of order $\beta^{-1}$.

We study the nonlinear evolution equation (1.1) on a finite time interval

$$
0 \leq \tau \leq \tau_{*} \text {, where } \tau_{*}>0 \text { is a fixed number }
$$

which may depend on the $L^{\infty}$ norm of the initial data $\mathbf{h}$ but, importantly, $\tau_{*}$ does not depend on $\varrho$. We consider classes of initial data such that wave evolution governed by (1.1) is significantly nonlinear on time interval $\left[0, \tau_{*}\right]$ and the effect of the nonlinearity $F(\mathbf{U})$ does not vanish as $\varrho \rightarrow 0$. We assume that $\beta$, $\varrho$ satisfy

$$
0<\beta \leq 1,0<\varrho \leq 1, \frac{\beta^{2}}{\varrho} \leq C_{1} \text { with some } C_{1}>0 .
$$

The above condition on the dispersion parameter $\frac{\beta^{2}}{\varrho}$ ensures that the dispersive effects are not dominant and do not suppress nonlinear effects, see [7] for a discussion.

To formulate the superposition principle more precisely we introduce first the solution operator $\mathcal{S}(\mathbf{h})(\tau): \mathbf{h} \rightarrow \mathbf{U}(\tau)$ which relates to the initial data $\mathbf{h}$ of the nonlinear evolution equation (1.1) the solution $\mathbf{U}(t)$ of this equation. Suppose that the initial state is a multiwavepacket, namely $\mathbf{h}=\sum \mathbf{h}_{l}$, with $\mathbf{h}_{l}, l=1, \ldots, N$ being "generic" wavepackets. Then for all times $0 \leq \tau \leq \tau_{*}$ the following superposition principle holds

$$
\begin{gathered}
\mathcal{S}\left(\sum_{l=1}^{N} \mathbf{h}_{l}\right)(\tau)=\sum_{l=1}^{N} \mathcal{S}\left(\mathbf{h}_{l}\right)(\tau)+\mathbf{D}(\tau), \\
\|\mathbf{D}(\tau)\|_{E}=\sup _{0 \leq \tau \leq \tau_{*}}\|\mathbf{D}(\tau)\|_{L^{\infty}} \leq C_{\delta} \frac{\varrho}{\beta^{1+\delta}} \text { for any small } \delta>0 .
\end{gathered}
$$

Obviously, the right-hand side of (1.13) may be small only if $\varrho \leq C_{1} \beta$. There are examples (see [7]) in which $\mathbf{D}(\tau)$ is not small for $\varrho=C_{1} \beta$. In what follows we refer to a linear 
combination of wavepackets as a multi-wavepacket, and to wavepackets which constitutes the multi-wavepacket as component wavepackets.

The superposition principle implies, in particular, that in the process of nonlinear evolution every single wavepacket propagates almost independently of other wavepackets even though they may "collide" in physical space for a certain period of time and the exact solution equals the sum of particular single wavepacket solutions with a high precision. In particular, the dynamics of a solution with multi-wavepacket initial data is reduced to dynamics of separate solutions with single wavepacket data. Note that the nonlinear evolution of a single wavepacket solution for many problems is studied in detail, namely it is well approximated by its own nonlinear Schrodinger equation (NLS), see [18, [23], 29], [30], 39], 40], 41], [7] and references therein.

The superposition principle (1.12), (1.13) can also be looked at as a form of separation of variables. Such a form of separation of variables is different from usual complete integrability, and its important factor is the continuity of spectrum of the linear component of the system. The approximate superposition principle imposes certain restrictions on dynamics which differ from usual constraints imposed by the conserved quantities as in completely integrable systems as well as from topological constraints related to invariant tori as in KAM theory.

Now we present an elementary physical argument justifying the superposition principle. If nonlinearity is absent, the superposition principle holds exactly and any deviation from it is due to the nonlinear interactions between wavepackets, so we need to estimate their impact. Suppose that initially at time $\tau=0$ the spatial extension $s$ of every composite wavepacket is characterized by the parameter $\beta^{-1}$ as in (1.9). Assume also (and it is quite an assumption) that the component wavepackets during the nonlinear evolution maintain somehow their wavepacket identity, group velocities and spatial extension. Then, consequently, the spatial extension of every component wavepacket is propositional to $\beta^{-1}$ and its group velocity $v_{j}$ is proportional to $\varrho^{-1}$. The difference $\Delta v$ between any two different component group velocities is also proportional to $\varrho^{-1}$. The time when two different component wavepackets overlap in space is proportional to $s /|\Delta v|$ and, hence, to $\varrho / \beta$. Since the nonlinear term is of order one, the magnitude of the impact of the nonlinearity during this time interval should be proportional to $\varrho / \beta$, which results in the same order of magnitude of $\mathbf{D}$. This conclusion is in agreement with the estimate of magnitude of $\mathbf{D}$ in (1.13) (if we set $\delta=0$ ).

The rigorous proof of the superposition principle we present in this paper is not based on the above argument since it implicitly relies on a superposition principle in the form of an assumption that component wavepackets can somehow maintain their identity, group velocities and spatial extension during nonlinear evolution which by no means is obvious. In fact, the question if a wavepacket or a multi-wavepacket structure can be preserved during nonlinear evolution is important and interesting question on its own right. The answer to it under natural conditions is affirmative as we have shown in [7]. Namely, if initially solution was a multi-wavepacket at $\tau=0$, it remains a multi-wavepacket at $\tau>0$, and every component wavepacket maintains its identity. Therefore a wavepacket can be interpreted as a quasi-particle which maintains its identity and can interact with other quasi-particles. This property holds also in the situation when there are stronger nonlinear interactions between 
wavepacket components which do not allow the superposition principle to hold, see [7] for details.

The proof we present here is based on general algebraic-functional considerations. The strategy of our proof is as follows. First, we prove that the operator $\mathcal{S}(\mathbf{h})$ in (1.12) is analytical, i.e. it can be written in the form of a convergent series

$$
\mathcal{S}(\mathbf{h})=\sum_{j=1}^{\infty} \mathcal{S}^{(j)}\left(\mathbf{h}^{j}\right), \mathbf{h}^{j}=\mathbf{h}, \ldots, \mathbf{h}(j \text { copies of } \mathbf{h}),
$$

where $\mathcal{S}^{(j)}\left(\mathbf{h}^{j}\right)$ is a $j$-linear operator applied to $\mathbf{h}$. Now we substitute $\mathbf{h}$ in $\mathcal{S}^{(j)}$ with the sum of $\mathbf{h}_{l}$ as in (1.2). Considering for simplicity the case $N=2$ and using the polylinearity of $\mathcal{S}^{(j)}$ we get

$$
\mathcal{S}^{(2)}\left(\left(\mathbf{h}_{1}+\mathbf{h}_{2}\right)^{2}\right)=\mathcal{S}^{(2)}\left(\left(\mathbf{h}_{1}\right)^{2}\right)+2 \mathcal{S}^{(2)}\left(\mathbf{h}_{1} \mathbf{h}_{2}\right)+\mathcal{S}^{(2)}\left(\left(\mathbf{h}_{2}\right)^{2}\right), \ldots,
$$

implying after the summation

$$
\begin{aligned}
\mathcal{S}(\mathbf{h}) & =\mathcal{S}^{(2)}\left(\left(\mathbf{h}_{1}\right)^{2}\right)+\mathcal{S}^{(3)}\left(\left(\mathbf{h}_{1}\right)^{3}\right)+\ldots+\mathcal{S}^{(2)}\left(\left(\mathbf{h}_{2}\right)^{2}\right)+\mathcal{S}^{(3)}\left(\left(\mathbf{h}_{2}\right)^{3}\right)+\ldots \mathcal{S}_{c r} \\
& =\mathcal{S}\left(\mathbf{h}_{1}\right)+\mathcal{S}\left(\mathbf{h}_{2}\right)+\mathcal{S}_{c r},
\end{aligned}
$$

where $\mathcal{S}_{c r}$ is a sum of all cross-terms such as $\mathcal{S}^{(2)}\left(\mathbf{h}_{1} \mathbf{h}_{2}\right)$ etc. The main part of the proof is to show that every term in $\mathcal{S}_{c r}$ is small. An important step for that is based on the refined implicit function theorem (Theorem 4.25) which allows to represent the operators $\mathcal{S}^{(j)}$ in the form of a sum of certain composition monomials, which, in turn, have a relatively simple oscillatory integral representation. Importantly, the relevant oscillatory integrals involve the known initial data $\mathbf{h}_{l}$ rather than unknown solution $\mathbf{U}$. The analysis of the oscillatory integrals shows that there are two mechanisms responsible for the smallness of the integrals. The first one is time averaging, and the second one is based on large group velocities (in the slow time scale) of wavepackets. Remarkably, if wavepackets satisfy proper genericity conditions, every cross term is small due one of the above mentioned two mechanisms. Importantly, the both mechanism are instrumental for the smallness of terms in $S_{c r}$, and the time averaging alone is not sufficient. We obtain estimates on terms in $\mathcal{S}_{c r}$ which ultimately yield the estimate (1.13). Since the smallness of interactions between waves under nonlinear evolution stems from high frequency oscillations in time and space of functions involved in the interaction integrals, we can interpret it as a result of the destructive wave interference. The above sketch shows that the mathematical tools we use in our studies are (i) the theory of analytic functions and corresponding series of infinite-dimensional (Banach) variable, and (ii) the theory of oscillatory integrals.

We would like to point out that the estimate (1.13) for the remainder in the superposition principle is quite accurate. For example, when the estimate is applied to the sine-Gordon equation with bimodal initial data, it yields essentially optimal estimates for the magnitude of the interaction of counterpropagating waves. These estimates are more accurate than ones obtained by the well known ansatz method as in [38], and the comparative analysis is provided below in Example 1, Section 2.2.

To summarize the above analysis we list important ingredients of our approach. 
- The spectrum of the underlying linear problem is continuous.

- The wave nonlinear evolution is analyzed based on the modal decomposition with respect to the linear component of the system because there is no exchange of energy between modes by linear mechanisms. Wavepacket definition is based on the modal expansion determining, in particular, its the spatial extension and the group velocity..

- The problem involves two small parameters $\beta$ and $\varrho$ respectively in the initial data and coefficients of the equations. These parameters scale respectively (i) the range of wavevectors involved in its modal composition, with $\beta^{-1}$ scaling its spatial extension, and (ii) $\varrho$ scaling the ratio of the slow and the fast time scales. We make no assumption on the functional dependence between $\beta$ and $\varrho$, which are essentially independent and are subject only to inequalities.

- The nonlinear evolution is studied for a finite time $\tau_{*}$ which may depend on, say, the amplitude of the initial excitation, and, importantly, $\tau_{*}$ is long enough to observe appreciable nonlinear phenomena which are not vanishingly small. The superposition principle can be extended to longer time intervals up to blow-up time or even infinity if relevant uniform in $\beta$ and $\varrho$ estimates of solutions in appropriate norms are available.

- Two fast wave processes (in the chosen slow time scale) attributed to the linear operator $\mathbf{L}$ and having typical time scale of order $\varrho$ can be identified as responsible for the essential independence of wavepackets: (i) fast time oscillations which lead to time averaging; (ii) fast wavepacket propagation with large group velocities produce effective weakening of interactions which are not subjected to time averaging.

The rest of the paper is organized as follows. In the following Section 2 we formulate exact conditions and theorems for lattice equations and partial differential equations and give examples. In Section 3 we recast the original evolution equation in a convenient reduced form allowing, in particular, to construct a representation of the solution in a form of convergent functional operator series explicitly involving the equation nonlinear term. In Section 4 we provide the detailed analysis of function-analytic series used to get a constructive representation of the solution. Section 5 is devoted to the analysis of certain oscillatory integrals which are terms of the series representing the solution. Note that when making estimations we use the same letter $C$ for different constants in different statements. Finally, the proofs of Theorems 2.15] and 2.19 are provided in Section 6. more examples and generalizations are given in Section 7. For reader's convenience we provide a list of notations in the end of the paper.

\section{Statement of results}

In this section we consider two classes of problems: lattice equations and partial differential equations. After Fourier transform they can be written in the modal form which is essentially 
the same in both cases. We formulate the exact conditions on the modal equations and present the main theorems on the superposition principle. We also give examples of equations to which the general theorems apply, in particular Fermi-Pasta-Ulam system and Nonlinear Schrodinger equation.

\subsection{Main definitions, statements and examples for the lattice equa- tion}

The first class of evolutionary systems we consider involves systems of equations describing coupled nonlinear oscillators on a lattice $\mathbb{Z}^{d}$, namely the following lattice system of ordinary differential equations (ODE's) with respect to time

$$
\partial_{\tau} \mathbf{U}(\mathbf{m}, \tau)=-\frac{\mathrm{i}}{\varrho} \mathbf{L} \mathbf{U}(\mathbf{m}, \tau)+F(\mathbf{U})(\mathbf{m}, \tau), \mathbf{U}(\mathbf{m}, 0)=\mathbf{h}(\mathbf{m}), \mathbf{m} \in \mathbb{Z}^{d},
$$

where $\mathbf{L}$ is a linear operator, $F$ is a nonlinear operator and $\varrho>0$ is a small parameter (see [6]). To analyze the evolution equation (2.1) it is instrumental to recast it in the modal form (the wavevector domain), in other words, to apply to it the lattice Fourier transform as defined by the formula

$$
\tilde{\mathbf{U}}(\mathbf{k})=\sum_{\mathbf{m} \in \mathbb{Z}^{d}} \mathbf{U}(\mathbf{m}) \mathrm{e}^{-\mathbf{i m} \cdot \mathbf{k}}, \text { where } \mathbf{k} \in[-\pi, \pi]^{d},
$$

$\mathbf{k}$ is called a wave vector. We assume that the Fourier transformation of the original lattice evolutionary equation (2.1) is of the form

$$
\partial_{\tau} \tilde{\mathbf{U}}(\mathbf{k}, \tau)=-\frac{\mathrm{i}}{\varrho} \mathbf{L}(\mathbf{k}) \tilde{\mathbf{U}}(\mathbf{k}, \tau)+\tilde{F}(\tilde{\mathbf{U}})(\mathbf{k}, \tau) ; \tilde{\mathbf{U}}(\mathbf{k}, 0)=\tilde{\mathbf{h}}(\mathbf{k}) \text { for } \tau=0 .
$$

Here, $\tilde{\mathbf{U}}(\mathbf{k}, \tau)$ is $2 J$ - component vector, $\mathbf{L}(\mathbf{k})$ is a $\mathbf{k}$-dependent $2 J \times 2 J$ matrix that corresponds to the linear operator $\mathbf{L}$ and $\tilde{F}(\tilde{\mathbf{U}})$ is a nonlinear operator, which we describe later. The matrix $\mathbf{L}(\mathbf{k})$ and the coefficients of the nonlinear operator $\tilde{F}(\tilde{\mathbf{U}})$ in (2.3) are $2 \pi$ periodic functions of $\mathbf{k}$ and for that reason we assume that $\mathbf{k}$ belongs to the torus $\mathbb{R}^{d} /(2 \pi \mathbb{Z})^{d}$ which we denote by $[-\pi, \pi]^{d}$. The $\mathbf{k}$-dependent matrix $\mathbf{L}(\mathbf{k})$ determines the linear operator $\mathbf{L}$ and plays an important role in the analysis. We refer to $\mathbf{L}(\mathbf{k})$ as to the linear symbol. Since (2.3) describes evolution of the Fourier modes of the solution, we call (2.3) modal evolution equation.

We study the modal evolution equation (2.3) on a finite time interval

$$
0 \leq \tau \leq \tau_{*}
$$

where $\tau_{*}>0$ is a fixed number which, as we will see, may depend on the magnitude of the initial data. The time $\tau_{*}$ does not depend on small parameters, it is of order one and is 
determined by norms of operators and initial data; it is almost optimal for general $F$ since there are examples when $\tau_{*}$ is of the same order as the blow up time of solutions. To make formulas and estimates simpler we assume without loss of generality that

$$
\tau_{*} \leq 1
$$

For a number of reasons the modal form (2.3) of the evolution equation is much more suitable for nonlinear analysis than the original evolution equation (2.1). This is why from now on we consider the modal form of evolution equation (2.3) for the modal components $\tilde{\mathbf{U}}(\mathbf{k}, \tau)$ as our primary evolution equation.

First, as an illustration, let us look at the simplest nontrivial example of (2.3) with $J=1$ corresponding to two-component vector fields on the lattice $\mathbb{Z}^{d}$. A two-component vector function $\mathbf{U}(\mathbf{m})$ of a discrete argument $\mathbf{m} \in \mathbb{Z}^{d}$ has the form

$$
\mathbf{U}(\mathbf{m})=\left[\begin{array}{c}
U_{+}(\mathbf{m}) \\
U_{-}(\mathbf{m})
\end{array}\right], \mathbf{m} \in \mathbb{Z}^{d} .
$$

In this example $\mathbf{L}(\mathbf{k})$ in $(2.3)$ is a $2 \times 2$ matrix, and we assume that for almost all $\mathbf{k}$ it has two different real eigenvalues $\omega_{-}(\mathbf{k})$ and $\omega_{+}(\mathbf{k})$ (the dependence of $\omega_{ \pm}(\mathbf{k})$ on $\mathbf{k}$ is called the dispersion relation) satisfying the relation $\omega_{-}(\mathbf{k})=-\omega_{+}(\mathbf{k})$, namely,

$$
\mathbf{L}(\mathbf{k}) \mathbf{g}_{\zeta}(\mathbf{k})=\omega_{\zeta}(\mathbf{k}) \mathbf{g}_{\zeta}(\mathbf{k}), \omega_{\zeta}(\mathbf{k})=\zeta \omega(\mathbf{k}), \zeta= \pm
$$

where, evidently, $\mathbf{g}_{\zeta}(\mathbf{k})$ are the eigenvectors of $\mathbf{L}(\mathbf{k})$. These eigenvalues $\omega_{\zeta}(\mathbf{k}), \zeta= \pm$, are $2 \pi$-periodic real valued functions

$$
\omega_{\zeta}\left(k_{1}+2 \pi, k_{2}, \ldots, k_{d}\right)=\ldots=\omega_{\zeta}\left(k_{1}, k_{2}, \ldots, k_{d}+2 \pi\right)=\omega_{\zeta}\left(k_{1}, k_{2}, \ldots, k_{d}\right) .
$$

The simplest nonlinearity in (2.3) is a quadratic nonlinear operator $\tilde{F}(\tilde{\mathbf{U}})=\tilde{F}^{(2)}\left(\tilde{\mathbf{U}}^{2}\right)$ which is given by the following convolution integral

$$
\tilde{F}^{(2)}\left(\tilde{\mathbf{U}}_{1} \tilde{\mathbf{U}}_{2}\right)(\mathbf{k})=\frac{1}{(2 \pi)^{d}} \int_{\mathbf{k}^{\prime} \in[-\pi, \pi]^{d} ; \mathbf{k}^{\prime}+\mathbf{k}^{\prime \prime}=\mathbf{k}} \chi^{(2)}(\mathbf{k}, \vec{k})\left(\tilde{\mathbf{U}}_{1}\left(\mathbf{k}^{\prime}\right) \tilde{\mathbf{U}}_{2}\left(\mathbf{k}^{\prime \prime}\right)\right) \mathrm{d} \mathbf{k}^{\prime}
$$

where $\vec{k}=\left(\mathbf{k}^{\prime}, \mathbf{k}^{\prime \prime}\right), \chi^{(2)}(\mathbf{k}, \vec{k})$ is a quadratic tensor (susceptibility) which acts on vectors $\tilde{\mathbf{U}}_{1}, \tilde{\mathbf{U}}_{2}$. We refer to the case $J=1$ as the one-band case since the corresponding linear operator is described by a single function $\omega(\mathbf{k})$.

A particular example of (2.3) is obtained as a Fourier transform of the following FermiPasta-Ulam equation (FPU) (see [12, 37], 44]) describing a nonlinear system of coupled oscillators:

$$
\begin{gathered}
\partial_{\tau} x_{n}=\frac{1}{\varrho}\left(y_{n}-y_{n-1}\right), \\
\partial_{\tau} y_{n}=\frac{1}{\varrho}\left(x_{n+1}-x_{n}\right)+\alpha_{2}\left(x_{n+1}-x_{n}\right)^{2}+\alpha_{3}\left(x_{n+1}-x_{n}\right)^{3}, n \in \mathbb{Z} .
\end{gathered}
$$


Note that an equivalent form of (2.10) (with $\left.\alpha_{2}=0\right)$ is the second order equation

$$
\partial_{\tau}^{2} x_{n}=\frac{1}{\varrho^{2}}\left(x_{n-1}-2 x_{n}+x_{n+1}\right)+\frac{\alpha_{3}}{\varrho}\left(\left(x_{n+1}-x_{n}\right)^{3}-\left(x_{n}-x_{n-1}\right)^{3}\right) .
$$

In this example $d=1, \mathbf{k}=k$ and elementary computations show that the Fourier transform of the FPU equation (2.10) has the form of the modal evolution equation (2.3), (2.9) where

$$
\begin{aligned}
& \tilde{\mathbf{U}}=\left[\begin{array}{c}
\tilde{x} \\
\tilde{y}
\end{array}\right], \mathrm{i} \mathbf{L}(k)=\left[\begin{array}{cc}
0 & -\left(1-\mathrm{e}^{-\mathrm{i} k}\right)^{*} \\
\left(1-\mathrm{e}^{-\mathrm{i} k}\right) & 0
\end{array}\right], \omega_{\zeta}(k)=2 \zeta\left|\sin \frac{k}{2}\right| \\
& \chi^{(2)}\left(k, k^{\prime}, k^{\prime \prime}\right) \tilde{\mathbf{U}}_{1}\left(k^{\prime}\right) \tilde{\mathbf{U}}_{2}\left(k^{\prime \prime}\right)=\alpha_{2}\left(1-\mathrm{e}^{-\mathrm{i} k^{\prime}}\right)\left(1-\mathrm{e}^{-\mathrm{i} k^{\prime \prime}}\right)\left[\begin{array}{c}
0 \\
\tilde{x}_{1}\left(k^{\prime}\right) \tilde{x}_{2}\left(k^{\prime \prime}\right)
\end{array}\right],
\end{aligned}
$$

and a similar formula for $\chi^{(3)}$ (see (7.5)).

Now let us consider the general multi-component vector case with $J>1$ which we refer to as $J$-band case for which the system (2.3) has $2 J$ components, and instead of (2.7) we assume that $\mathbf{L}(\mathbf{k})$ has eigenvalues and eigenvectors as follows:

$$
\mathbf{L}(\mathbf{k}) \mathbf{g}_{n, \zeta}(\mathbf{k})=\omega_{n, \zeta}(\mathbf{k}) \mathbf{g}_{n, \zeta}(\mathbf{k}), \omega_{n, \zeta}(\mathbf{k})=\zeta \omega_{n}(\mathbf{k}), \zeta= \pm, n=1, \ldots, J
$$

where $\omega_{n}(\mathbf{k})$ are real-valued, continuous for all $\mathbf{k}$ functions, and eigenvectors $\mathbf{g}_{n, \zeta}(\mathbf{k}) \in \mathbb{C}^{2 J}$ have unit length in the standard Euclidean norm. We also suppose that the eigenvalues are numbered so that

$$
\omega_{n+1}(\mathbf{k}) \geq \omega_{n}(\mathbf{k}) \geq 0, n=1, \ldots, J-1,
$$

and we call $n$ the band index. Note that the presence of $\zeta= \pm$ reflects a symmetry of the system allowing it, in particular, to have real-valued solutions. Such a symmetry of dispersion relation $\omega_{n}(\mathbf{k})$ occurs in photonic crystals and many other physical problems.

Note that (2.13) implies that the following symmetry relation hold:

$$
\omega_{n,-\zeta}(\mathbf{k})=-\omega_{n, \zeta}(\mathbf{k}), n=1, \ldots, J
$$

We also always assume that the following inversion symmetry holds:

$$
\omega_{n, \zeta}(-\mathbf{k})=\omega_{n, \zeta}(\mathbf{k})
$$

Remark 2.1 Assuming (2.15) and (2.16) we suppose that the dispersion relations $\omega_{\zeta}(\mathbf{k})$ have the same symmetry properties as the dispersion relations of Maxwell equations in periodic media, see [1]-[3], [5]. We would like to stress that these symmetry conditions are not imposed for technical reasons but because they are consequences of fundamental symmetries of physical media. Such symmetries arise in many problems including, for instance, the Fermi-PastaUlam equation, or when $\mathbf{L}(\mathbf{k})$ originates from a Hamiltonian $H(p, q)=\frac{1}{2}\left(H_{1}\left(p^{2}\right)\right)+\frac{1}{2} H_{2}\left(q^{2}\right)$. In the opposite case if it is assumed that (2.15) and (2.16) never hold, the results of this paper hold and the proofs, in fact, are simpler. The case with the symmetry is more difficult and delicate because of a possibility of resonant nonlinear interactions. 
There are values of $\mathbf{k}$ for which inequalities (2.14) turn into equalities, these points require special treatment.

Definition 2.2 (band-crossing points) We call $\mathbf{k}_{0}$ a band-crossing point if $\omega_{n+1}\left(\mathbf{k}_{0}\right)=$ $\omega_{n}\left(\mathbf{k}_{0}\right)$ for some $n$ or $\omega_{1}\left(\mathbf{k}_{0}\right)=0$ and denote the set of band-crossing points by $\sigma$.

Everywhere in this paper we assume that the following condition is satisfied.

Condition 2.3 The set $\sigma$ of band-crossing points is a closed nowhere dense set in $\mathbb{R}^{d}$ with zero Lebesgue measure, the entries of the matrix $\mathbf{L}(\mathbf{k})$ are infinitely smooth functions of $\mathbf{k} \notin \sigma$ and $\omega_{n}(\mathbf{k})$ are continuous functions of $\mathbf{k}$ for all $\mathbf{k}$ and are infinitely smooth when $\mathbf{k} \notin \sigma$.

Observe that for $\mathbf{k} \notin \sigma$ all the eigenvalues of the matrix $\mathbf{L}(\mathbf{k})$ are different and the corresponding eigenvectors $\mathbf{g}_{n, \zeta}(\mathbf{k})$ of $\mathbf{L}(\mathbf{k})$ can be locally defined as smooth functions of $\mathbf{k} \notin \sigma$ as long as $\mathbf{L}(\mathbf{k})$ is smooth.

Remark 2.4 The band-crossing points are discussed in more details in [1], [2]. Here we only note that generically the singular set $\sigma$ is a manifold of the dimension $d-2$, see [1], [2]). A simple example of a band-crossing point is $k=0$ in (2.12).

Since we do not assume the matrix $\mathbf{L}(\mathbf{k})$ to be Hermitian, we impose the following condition on its eigenfunctions which guarantees its uniform diagonalization.

Condition 2.5 We assume that the $2 J \times 2 J$ matrix formed by the eigenvectors $\mathbf{g}_{n, \zeta}(\mathbf{k})$ of $\mathbf{L}(\mathbf{k})$, namely,

$$
\Xi(\mathbf{k})=\left[\mathbf{g}_{1,+}(\mathbf{k}), \mathbf{g}_{1,-}(\mathbf{k}), \ldots, \mathbf{g}_{J,+}(\mathbf{k}), \mathbf{g}_{J,+}(\mathbf{k})\right]
$$

is uniformly bounded together with its inverse

$$
\sup _{\mathbf{k} \notin \sigma}\|\Xi(\mathbf{k})\|, \sup _{\mathbf{k} \notin \sigma}\left\|\Xi^{-1}(\mathbf{k})\right\| \leq C_{\Xi} \text { for some constant } C_{\Xi} .
$$

Here and everywhere we use the standard Euclidean norm in $\mathbb{C}^{2 J}$.

Note that if the matrix $\mathbf{L}(\mathbf{k})$ is Hermitian for every $\mathbf{k}$, the eigenvectors form an orthonormal system. Then the matrix $\Xi$, which diagonalizes $\mathbf{L}$, is unitary and (2.17) is satisfied with $C_{\Xi}=1$. Everywhere throughout the paper we assume that Condition 2.5 is satisfied.

We introduce for vectors $\tilde{\mathbf{u}} \in \mathbb{C}^{2 J}$ their expansion with respect to the basis $\mathbf{g}_{n, \zeta}$ :

$$
\tilde{\mathbf{u}}(\mathbf{k})=\sum_{n=1}^{J} \sum_{\zeta= \pm} \tilde{u}_{n, \zeta}(\mathbf{k}) \mathbf{g}_{n, \zeta}(\mathbf{k})=\sum_{n=1}^{J} \sum_{\zeta= \pm} \tilde{\mathbf{u}}_{n, \zeta}(\mathbf{k})
$$

and we refer to it as the modal decomposition of $\tilde{\mathbf{u}}(\mathbf{k})$, and call the coefficients $\tilde{u}_{n, \zeta}(\mathbf{k})$ the modal coefficients of $\tilde{\mathbf{u}}(\mathbf{k})$. In this expansion we assign to every $n, \zeta$ a linear projection $\Pi_{n, \zeta}(\mathbf{k})$ in $\mathbb{C}^{2 J}$ corresponding to $\mathbf{g}_{n, \zeta}(\mathbf{k})$, namely

$$
\Pi_{n, \zeta}(\mathbf{k}) \tilde{\mathbf{u}}(\mathbf{k})=\tilde{u}_{n, \zeta}(\mathbf{k}) \mathbf{g}_{n, \zeta}(\mathbf{k})=\tilde{\mathbf{u}}_{n, \zeta}(\mathbf{k}), n=1, \ldots, J, \zeta= \pm .
$$


Note that these projections may be not orthogonal if $\mathbf{L}(\mathbf{k})$ is not Hermitian. Evidently the projections $\Pi_{n, \zeta}(\mathbf{k})$ are determined by the matrix $\mathbf{L}(\mathbf{k})$ and therefore do not depend on the choice of the basis $\mathbf{g}_{n, \zeta}(\mathbf{k})$. Projections $\Pi_{n, \zeta}(\mathbf{k})$ depend smoothly on $\mathbf{k} \notin \sigma$ (note that we do not assume that the basis elements $\mathbf{g}_{n, \zeta}(\mathbf{k})$ are defined globally as smooth functions for all $\mathbf{k} \notin \sigma$, in fact band-crossing points may be branching points for eigenfunctions, see for example [1.) They are also uniformly bounded thanks to Condition 2.5.

$$
C_{\Xi}^{-1}|\mathbf{V}| \leq\left(\sum_{n, \zeta}\left|\Pi_{n, \zeta}(\mathbf{k}) \mathbf{V}\right|^{2}\right)^{1 / 2} \leq C_{\Xi}|\mathbf{V}|, \quad \mathbf{V} \in \mathbb{C}^{2 J}, \mathbf{k} \notin \sigma .
$$

We would like to point out that most of the quantities are defined outside of the singular set $\sigma$ of band-crossing points. It is sufficient since we consider $\tilde{\mathbf{U}}(\mathbf{k})$ as an element of the space $L_{1}$ of Lebesgue integrable functions and the set $\sigma$ has zero Lebesgue measure.

The class of nonlinearities $\tilde{F}$ in (2.3) which we consider can be described as follows. $\tilde{F}$ is a general polynomial nonlinearity of the form

$$
\tilde{F}(\tilde{\mathbf{U}})=\sum_{m=2}^{m_{F}} \tilde{F}^{(m)}\left(\tilde{\mathbf{U}}^{m}\right), \text { with } m_{F} \geq 2,
$$

where $m$-linear operators $\tilde{F}^{(m)}$ are represented by integral convolution formulas similar to (2.9), namely

$$
\tilde{F}^{(m)}\left(\tilde{\mathbf{U}}_{1}, \ldots, \tilde{\mathbf{U}}_{m}\right)(\mathbf{k}, \tau)=\int_{\mathbb{D}_{m}} \chi^{(m)}(\mathbf{k}, \vec{k}) \tilde{\mathbf{U}}_{1}\left(\mathbf{k}^{\prime}\right) \ldots \tilde{\mathbf{U}}_{m}\left(\mathbf{k}^{(m)}(\mathbf{k}, \vec{k})\right) \tilde{\mathrm{d}}^{(m-1) d} \vec{k}
$$

where the domain

$$
\mathbb{D}_{m}=[-\pi, \pi]^{(m-1) d}
$$

and we use notation

$$
\tilde{\mathrm{d}}^{(m-1) d} \vec{k}=\frac{1}{(2 \pi)^{(m-1) d}} \mathrm{~d} \mathbf{k}^{\prime} \ldots \mathrm{d} \mathbf{k}^{(m-1)}
$$

and

$$
\mathbf{k}^{(m)}(\mathbf{k}, \vec{k})=\mathbf{k}-\mathbf{k}^{\prime}-\ldots-\mathbf{k}^{(m-1)}, \vec{k}=\left(\mathbf{k}^{\prime}, \ldots, \mathbf{k}^{(m)}\right)
$$

Condition 2.6 (nonlinearity regularity) The nonlinear operator $\tilde{F}(\tilde{\mathbf{U}})$ defined by (2.21) satisfy

$$
\left\|\chi^{(m)}\right\|=\frac{1}{(2 \pi)^{(m-1) d}} \sup _{\mathbf{k}, \mathbf{k}^{\prime}, \ldots, \mathbf{k}^{(m)}}\left\|\chi^{(m)}\left(\mathbf{k}, \mathbf{k}^{\prime}, \ldots, \mathbf{k}^{(m)}\right)\right\| \leq C_{\chi}, m=2,3, \ldots,
$$

where, without loss of generality, we can assume that $C_{\chi} \geq 1$. The norm $\left|\chi^{(m)}(\mathbf{k}, \vec{k})\right|$ of the tensor $\chi^{(m)}$ with a fixed $\vec{k}$ as a m-linear operator from $\left(\mathbb{C}^{2 J}\right)^{m}$ into $\left(\mathbb{C}^{2 J}\right)$ is defined by

$$
\left|\chi^{(m)}(\mathbf{k}, \vec{k})\right|=\sup _{\left|\mathbf{x}_{j}\right| \leq 1}\left|\chi^{(m)}(\mathbf{k}, \vec{k})\left(\mathbf{x}_{1}, \ldots, \mathbf{x}_{m}\right)\right|
$$


where as always, $|\cdot|$ stands for the standard Euclidean norm. The tensors $\chi^{(m)}(\mathbf{k}, \vec{k})$ are assumed to be smooth functions of $\mathbf{k}, \mathbf{k}^{\prime}, \ldots, \mathbf{k}^{(m)} \notin \sigma$, namely for every compact $K \subset \mathbb{R}^{d} \backslash \sigma$ and for all $m=2,3, \ldots$.

$$
\left|\nabla^{l} \chi^{(m)}\left(\mathbf{k}, \mathbf{k}^{\prime}, \ldots, \mathbf{k}^{(m)}\right)\right| \leq C_{K, l} \text { if } \mathbf{k}, \mathbf{k}^{\prime}, \ldots, \mathbf{k}^{(m)} \in K, l=1,2, \ldots,
$$

where $\nabla^{l} \chi^{(m)}$ is the vector composed of all partial derivatives of order $l$ of all components of the tensor $\chi^{(m)}$ with respect to the variables $\mathbf{k}, \mathbf{k}^{\prime}, \ldots, \mathbf{k}^{(m)}$.

¿From now on all the nonlinear operators we consider are assumed to satisfy the nonlinearity regularity Condition 2.6 .

Remark 2.7 At first sight, since @ is a small parameter, one might think that the linear term in (2.1) with the factor $\frac{1}{\varrho}$ is dominant. But it is not that simple. Indeed, since all eigenvalues of $\mathbf{L}(\mathbf{k})$ are purely imaginary the magnitude of $\mathrm{e}^{-\frac{i}{\varrho} \mathbf{L}(\mathbf{k})} \tilde{\mathbf{h}}(\mathbf{k})$ which represents the solution of a linear equation (with $\tilde{F}=0$ ) is bounded uniformly in $\varrho$. A nonlinearity $\tilde{F}$ alters the solution for a bounded time $\tau_{*}$ which is not small for small $\varrho$. Therefore the influence of the nonlinearity can be significant. This phenomenon can be illustrated by the following toy model. Let us consider the partial differential equation for a scalar function $y(x, \tau)$ :

$$
\partial_{\tau} y=-\frac{1}{\varrho} \partial_{x} y+y^{2}, y(x, 0)=h(x) .
$$

Its solution is of the form

$$
y(x, \tau)=\frac{h\left(x-\frac{\tau}{\varrho}\right)}{1-\tau h\left(x-\frac{\tau}{\varrho}\right)},
$$

and regularly it exists only for a finite time. The solution (2.29) shows that the large coefficient $\frac{1}{\varrho}$ enters it so that the corresponding wave moves faster with the velocity $\frac{1}{\varrho}$ along the $x$-axis but the wave's shape does not depend on $\varrho$ at all. For the NLS with the initial data $\tilde{\mathbf{h}}(\mathbf{k})=\tilde{\mathbf{h}}(\mathbf{k}, \beta), \varrho=\beta^{2}$, and the coefficient $\frac{1}{\varrho}$ at the linear part, the nonlinearity balances the effect of dispersion leading to emergence of solitons, see [6] for a discussion.

To formulate our results we introduce a Banach space $E=C\left(\left[0, \tau_{*}\right], L_{1}\right)$ of functions $\tilde{\mathbf{v}}(\mathbf{k}, \tau), 0 \leq \tau \leq \tau_{*}$, with the norm

$$
\|\tilde{\mathbf{v}}(\mathbf{k}, \tau)\|_{E}=\|\tilde{\mathbf{v}}(\mathbf{k}, \tau)\|_{C\left(\left[0, \tau_{*}\right], L_{1}\right)}=\sup _{0 \leq \tau \leq \tau_{*}} \int_{[-\pi, \pi]^{d}}|\tilde{\mathbf{v}}(\mathbf{k}, \tau)| \mathrm{d} \mathbf{k} .
$$

Here $L_{1}$ is the Lebesgue function space with the standard norm defined by the formula

$$
\|\tilde{\mathbf{v}}(\cdot)\|_{L_{1}}=\int_{[-\pi, \pi]^{d}}|\tilde{\mathbf{v}}(\mathbf{k})| \mathrm{d} \mathbf{k} .
$$

The following theorem guarantees the existence and the uniqueness of a solution to the modal evolution equation (2.3) on a time interval which does not depend on $\varrho$ (see Theorem 5.4 for details). 
Theorem 2.8 (existence and uniqueness) Let the modal evolution equation (2.3) satisfy the Condition 2.5, and let $\tilde{\mathbf{h}} \in L_{1},\|\tilde{\mathbf{h}}\|_{L_{1}} \leq R$. Then there exists a unique solution $\tilde{\mathbf{U}}=$ $\mathcal{G}(\tilde{\mathbf{h}})$ of (2.3) which belongs to $C^{1}\left(\left[0, \tau_{*}\right], L_{1}\right)$. The number $\tau_{*}>0$ depends on $R, C_{\chi}$ and $C_{\Xi}$ and it does not depend on $\varrho$.

Now we would like to formulate the main result of this paper, a theorem on the superposition principle, showing that the generic wavepackets evolve almost independently for the case of lattice equations. To do that, first, we define an important concept of wavepacket.

Definition 2.9 (wavepacket) A function $\tilde{\mathbf{h}}(\beta, \mathbf{k})$ which depends on a parameter $0<\beta<$ 1 , is called a wavepacket with a center $\mathbf{k}_{*}$ if it satisfies the following conditions:

(i) It is bounded in $L_{1}$ uniformly in $\beta$, i.e.

$$
\|\tilde{\mathbf{h}}(\beta, \cdot)\|_{L_{1}} \leq C_{h}
$$

(ii) It is composed of modes from essentially a single band $n$, namely for any $0<\epsilon<1$ there is a constant $C_{\epsilon}>0$ such that

$$
\left\|\tilde{\mathbf{h}}(\mathbf{k})-\tilde{\mathbf{h}}_{-}(\mathbf{k})-\tilde{\mathbf{h}}_{+}(\mathbf{k})\right\|_{L_{1}} \leq C_{\epsilon} \beta, \tilde{\mathbf{h}}_{\zeta}(\mathbf{k})=\Pi_{n, \zeta} \tilde{\mathbf{h}}(\mathbf{k}), \zeta= \pm
$$

and $\tilde{\mathbf{h}}_{\zeta}(\beta, \mathbf{k})$ is essentially supported in a small vicinity of $\zeta \mathbf{k}_{*}$, where $\mathbf{k}_{*}$ is the wavepacket center, namely

$$
\int_{\left|\mathbf{k}-\zeta \mathbf{k}_{*}\right| \geq \beta^{1-\epsilon}}\left|\tilde{\mathbf{h}}_{\zeta}(\beta, \mathbf{k})\right| \mathrm{d} \mathbf{k} \leq C_{\epsilon} \beta .
$$

(iii) The wavepacket center $\mathbf{k}_{*}$ is not a band-crossing point, that is $\mathbf{k}_{* l} \notin \sigma$, and the following regularity condition holds:

$$
\int_{\left|\mathbf{k}-\zeta \mathbf{k}_{*}\right| \leq \beta^{1-\epsilon}}\left|\nabla_{\mathbf{k}} \tilde{\mathbf{h}}_{\zeta}(\beta, \mathbf{k})\right| \mathrm{d} \mathbf{k} \leq C_{\epsilon} \beta^{-1-\epsilon} .
$$

In the above conditions (ii) and (iii) $C_{\epsilon}$ does not depend on $\beta, 0<\beta<1$.

The simplest example of a wavepacket in the sense of Definition 2.9 is a function of the form

$$
\tilde{\mathbf{h}}_{\zeta}(\beta, \mathbf{k})=\beta^{-d} \hat{h}_{\zeta}\left(\frac{\mathbf{k}-\zeta \mathbf{k}_{*}}{\beta}\right) \mathbf{g}_{n, \zeta}(\mathbf{k}), \zeta= \pm
$$

where $\hat{h}_{\zeta}(\mathbf{k})$ is a Schwartz function, that is an infinitely smooth, rapidly decaying function. Another typical and natural example of a wavepacket $\tilde{\mathbf{h}}$ centered at $\mathbf{k}_{*}$ is readily provided by

$$
\tilde{\mathbf{h}}(\beta, \mathbf{k})=\Pi_{n,+}(\mathbf{k}) \tilde{\mathbf{h}}_{0,+}(\beta, \mathbf{k})+\Pi_{n,-}(\mathbf{k}) \tilde{\mathbf{h}}_{0,-}(\beta, \mathbf{k})
$$


where $\tilde{\mathbf{h}}_{0, \zeta}(\beta, \mathbf{k})$ is the lattice Fourier transform of the following function

$$
\mathbf{h}_{0, \zeta}(\mathbf{m}, \beta)=\mathrm{e}^{\mathrm{i} \zeta \mathbf{k}_{*} \cdot \mathbf{m}} \Phi_{\zeta}\left(\beta \mathbf{m}-\mathbf{r}_{0}\right) \mathbf{g}, \zeta= \pm,
$$

where $\mathbf{g}$ is a vector in $\mathbb{C}^{2 J}$, projection $\Pi_{n, \zeta}$ is as in (2.19) with some $n$, vectors $\mathbf{m}, \mathbf{r}_{0} \in \mathbb{R}^{d}$ and $\Phi_{\zeta}(\mathbf{r})$ being an arbitrary Schwartz function (see Lemma 17.2).

Our special interest is in the waves that are finite sums of wavepackets and we refer to them as multi-wavepackets.

Definition 2.10 (multi-wavepacket) A function $\tilde{\mathbf{h}}(\beta, \mathbf{k}), 0<\beta<1$, is called a multiwavepacket if it is a finite sum of wavepackets $\tilde{\mathbf{h}}_{l}$ as defined in Definition 2.9, namely

$$
\tilde{\mathbf{h}}(\beta, \mathbf{k})=\sum_{l=1}^{N_{h}} \tilde{\mathbf{h}}_{l}(\beta, \mathbf{k}),
$$

and we call the set $\left\{\mathbf{k}_{* l}\right\}$ of all the centers $\mathbf{k}_{* l}$ of involved wavepackets center set of $\tilde{\mathbf{h}}$.

In what follows we will be interested in generic multi-wavepackets such that their centers are generic. The exact meaning of this is provided below in the following conditions.

Condition 2.11 (non-zero frequency) We assume that every center $\mathbf{k}_{* l}$ of a wavepacket satisfies the following condition

$$
\omega_{n_{l}}\left(\mathbf{k}_{* l}\right) \neq 0, l=1, \ldots, N_{h} .
$$

Condition 2.12 (group velocity) We assume that all centers $\mathbf{k}_{* l}, l=1, \ldots, N_{h}$, of the multi-wavepacket $\tilde{\mathbf{h}}$ as defined in Definition 2.10 are not band-crossing points, and the gradients $\nabla_{\mathbf{k}} \omega_{n_{l_{j}}}\left(\mathbf{k}_{* l_{j}}\right)$ (called group velocities) at these points satisfy the following condition

$$
\left|\nabla_{\mathbf{k}} \omega_{n_{l_{1}}}\left(\mathbf{k}_{* l_{1}}\right)-\nabla_{\mathbf{k}} \omega_{n_{l_{2}}}\left(\mathbf{k}_{* l_{2}}\right)\right| \neq 0 \text { when } l_{1} \neq l_{2},
$$

indicating that the group velocities are different.

We also want the functions (dispersion relations) $\omega_{n_{l}}(\mathbf{k})$ to be non-degenerate in the sense that they are not exactly linear, below we give exact conditions.

Consider the following equation for $n$ and $\theta$

$$
\theta \omega_{n_{l}}\left(\mathbf{k}_{*}\right)-\zeta \omega_{n}\left(\theta \mathbf{k}_{*}\right)=0, \quad \zeta= \pm 1
$$

where the admissible $\theta$ have the form

$$
\theta=\sum_{j=1}^{m} \zeta^{(j)}, \zeta^{(j)}= \pm 1, m \leq m_{F},
$$

$m_{F}$ is the same as in (2.21). In the case when in the series (2.21) some terms $\tilde{F}^{(m)}$ vanish, we take in (2.43) only $m$ corresponding to non-zero $\tilde{F}^{(m)}$. 
Condition 2.13 (non-degeneracy) Given a point $\mathbf{k}_{*}=\mathbf{k}_{* l}$ and band $n_{l}$ we assume that dispersion relations $\omega_{n}(\mathbf{k})$ are such that all solutions $n, \theta$ of 2.42$)$ are necessarily of the form

$$
n=n_{l}, \theta=\zeta .
$$

Definition 2.14 (generic multi-wavepackets) A multi-wavepacket $\tilde{\mathbf{h}}$ as defined in Definition 2.10 is called generic if the centers $\mathbf{k}_{* l}, l=1, \ldots, N_{h}$, of all wavepackets satisfy Conditions [2.11 and 2.12; and the dispersion relations $\omega_{n}(\mathbf{k})$ at every $\mathbf{k}_{* l}$ and band $n_{l}$ satisfy Condition 2.13.

We introduce now the solution operator $\mathcal{G}$ mapping the initial data $\tilde{\mathbf{h}}$ into the solution $\tilde{\mathbf{U}}=\mathcal{G}(\tilde{\mathbf{h}})$ of the modal evolution equation (2.3); this operator is defined for $\|\tilde{\mathbf{h}}\| \leq R$ according to Theorem 2.8. The main result of this paper for the lattice case is the following statement.

Theorem 2.15 (superposition principle for lattice equations) Suppose that the initial data $\tilde{\mathbf{h}}$ of (2.3) is a multi-wavepacket of the form

$$
\tilde{\mathbf{h}}=\sum_{l=1}^{N_{h}} \tilde{\mathbf{h}}_{l}, N_{h} \max _{l}\left\|\tilde{\mathbf{h}}_{l}\right\|_{L_{1}} \leq R
$$

satisfying Definition 2.10, where $\tilde{\mathbf{h}}$ is generic in the sense of Definition 2.14. Let us assume that

$$
\frac{\beta^{2}}{\varrho} \leq C \text {, with some } C, \quad 0<\beta \leq \frac{1}{2}, 0<\varrho \leq \frac{1}{2} .
$$

Then the solution $\tilde{\mathbf{U}}=\mathcal{G}(\tilde{\mathbf{h}})$ to the evolution equation (2.3) satisfies the following approximate superposition principle

$$
\mathcal{G}\left(\sum_{l=1}^{N_{h}} \tilde{\mathbf{h}}_{l}\right)=\sum_{l=1}^{N_{h}} \mathcal{G}\left(\tilde{\mathbf{h}}_{l}\right)+\tilde{\mathbf{D}},
$$

with a small remainder $\tilde{\mathbf{D}}(\tau)$ satisfying the following estimate

$$
\sup _{0 \leq \tau \leq \tau_{*}}\|\tilde{\mathbf{D}}(\tau)\|_{L_{1}} \leq C_{\epsilon} \frac{\varrho}{\beta^{1+\epsilon}}|\ln \beta|,
$$

where $\epsilon$ is the same as in Definition 2.9 and can be arbitrary small, $\tau_{*}$ does not depend on $\beta, \varrho$ and $\epsilon$.

The most common case when (2.46) holds is $\varrho=\beta^{2}$, a discussion of different scalings is provided in [6] and [7].

Observe that solutions to the original evolution equation (2.1) with the initial data (2.39), (2.38) satisfy the superposition principle if the wave vectors $\mathbf{k}_{* l}$ in (2.38) satisfy (2.41), (2.42) 
and $\Phi_{l}$ are Schwartz functions. It turns out, that the evolution of every coefficient $\tilde{u}_{n, \zeta}(\mathbf{k})$ of the solution as defined by (2.18) can be accurately approximated by a solution a relevant Nonlinear Schrodinger equation (NLS), see 23. Therefore Theorem 2.15 provides a reduction of multi-wavepacket problem to several single-wavepacket problems.

We also would like to stress that though $\beta$ is small the nonlinear effects are not small. Namely, there can be a significant difference between solutions of a nonlinear and the corresponding linear (with $F(\mathbf{U})$ being set zero) equations with the same initial data for times $\tau=\tau_{*}$.

Recall that up to now we analyzed the nonlinear evolution in the modal form (2.3) for $\tilde{\mathbf{U}}(\mathbf{k}, \tau)$. To make a statement on the nonlinear evolution for the original evolution equation (2.1), i.e. in terms of the quantities $\mathbf{U}(\mathbf{m}, \tau)$, we introduce $\mathbf{U}(\mathbf{h})(\mathbf{m})$ as the inverse Fourier transform of the solution $\mathcal{G}(\tilde{\mathbf{h}})(\mathbf{k})$ of the modal evolution equation (2.3). Recall that the inverse Fourier transform corresponding to (2.2) is given by the formula

$$
\mathbf{U}(\mathbf{m})=(2 \pi)^{-d} \int_{[-\pi, \pi]^{d}} \mathrm{e}^{\mathrm{i} \mathbf{m} \cdot \mathbf{k}} \tilde{\mathbf{U}}(\mathbf{k}) \mathrm{d} \mathbf{k},
$$

and when applying the inverse Fourier transform we get back the original lattice system (2.1) from its modal form (2.3). The convolution form of the nonlinearity makes the lattice system invariant with respect to translations on the lattice $\mathbb{Z}^{d}$. Using Theorem 2.15 and applying the inverse Fourier transform together with the inequality

$$
\|\mathbf{U}\|_{L_{\infty}} \leq(2 \pi)^{-d}\|\tilde{\mathbf{U}}\|_{L_{1}}
$$

we obtain the following statement.

Corollary 2.16 Let the evolution equation (2.1) be obtained as the lattice Fourier transform of (2.3). If $\mathbf{h}$ is given by (2.38) where every $\Phi_{l, \zeta}(\mathbf{r})$ is a Schwartz function (that is an infinitely smooth, rapidly decaying function) then $\mathbf{U}(\mathbf{h})$ is a solution to the evolution equation (2.1). If $\mathbf{h}=\mathbf{h}_{1}+\ldots+\mathbf{h}_{N_{h}}$ and every $\mathbf{h}_{l}$ is given by (2.38) then the approximate superposition principle holds:

$$
\mathbf{U}(\mathbf{h})=\mathbf{U}\left(\mathbf{h}_{1}\right)+\ldots+\mathbf{U}\left(\mathbf{h}_{N_{h}}\right)+\mathbf{D}
$$

with a small coupling remainder $\mathbf{D}(\tau)$ satisfying

$$
\sup _{0 \leq \tau \leq \tau_{*}}\|\mathbf{D}(\tau)\|_{L_{\infty}} \leq C_{\delta}^{\prime} \frac{\varrho}{\beta^{1+\delta}}
$$

where $\delta>0$ can be taken arbitrary small.

As an application of Theorem 2.15 let us consider the Fermi-Pasta-Ulam equation (2.10). We impose the initial condition for (2.10)

$$
x_{n}(0)=\sum_{l=1}^{n_{h}} \Psi_{0 l}\left(\beta n-r_{l}\right) \mathrm{e}^{\mathrm{i} \mathbf{k}_{* l} n}+\mathrm{cc}, y_{n}(0)=\sum_{l=1}^{n_{h}} \Psi_{1 l}\left(\beta n-r_{l}\right) \mathrm{e}^{\mathrm{i} \mathbf{k}_{* l} n}+\mathrm{cc}, n \in \mathbb{Z},
$$


where $\Psi_{0 l}(r), \Psi_{1 l}(r)$ are arbitrary Schwartz functions, and $r_{l}$ are arbitrary real numbers, cc means complex conjugate to the preceding terms and assume that $\varrho, \beta$ satisfy (2.46). For any given $k_{* l}$ there are two eigenvectors $\mathbf{g}_{ \pm}\left(k_{* l}\right)$ of the matrix $\mathbf{L}\left(k_{* l}\right)$ in (2.12) given by (7.3) and corresponding terms in (2.53) can be written as

$$
\left[\begin{array}{l}
\Psi_{0 l} \\
\Psi_{1 l}
\end{array}\right] \mathrm{e}^{\mathrm{i} \mathbf{k}_{* l} n}=\left[\Phi_{-, l} \mathbf{g}_{-}\left(k_{* l}\right)+\Phi_{+, l} \mathbf{g}_{+}\left(k_{* l}\right)\right] \mathrm{e}^{\mathrm{i} \mathbf{k}_{* l} n} .
$$

In this case all requirements of Definition 2.10 are fulfilled, and (2.53) defines a multiwavepacket. Note that the multiwavepacket (2.53) involves $N_{h}=2 n_{h}$ wavepackets with $2 n_{h}$ wavepacket centers $\vartheta k_{* l}, \vartheta= \pm$. To satisfy Condition 2.12 the wavepacket centers $k_{* l}$ must satisfy

$$
\frac{\cos \frac{k_{* l}}{2}}{\left|\sin \frac{k_{* l}}{2}\right|} \neq \frac{\cos \frac{k_{* j}}{2}}{\left|\sin \frac{k_{* j}}{2}\right|} \text { if } l \neq j
$$

To check if the centers $k_{* l}$ satisfy Condition 2.13 we consider the equation

$$
z\left|\sin \frac{k_{* l}}{2}\right|-\zeta\left|\sin \left(z \frac{k_{* l}}{2}\right)\right|=0, z=\sum_{j=1}^{3} \zeta^{(j)}, \zeta^{(j)}= \pm 1 .
$$

Evidently the possible values of $z$ are $-3,-1,1,3$. Since the equation $3|\sin \phi|=|\sin (3 \phi)|$ has the only solution $\phi=0$ on $[0, \pi / 2]$ the equation (2.55) has the only solution $z=\zeta$. Consequently, all points $k_{* l} \neq 0$ satisfy Condition 2.13, and Theorem 2.15 applies. The initial data for a single wavepacket solution have the form

$$
\left[\begin{array}{l}
x_{\vartheta, n, l}(0) \\
y_{\vartheta, n, l}(0)
\end{array}\right]=\Phi_{\vartheta, l}\left(\beta n-r_{l}\right) \mathbf{g}_{\vartheta}\left(k_{* l}\right)+\mathrm{cc}, n \in \mathbb{Z}, \vartheta= \pm .
$$

According to this theorem and Corollary 2.16] the solution to (2.10), (2.53) equals the sum of solutions of (2.10) with single wavepacket initial data, that is

$$
x_{n}(\tau)=\sum_{\vartheta= \pm} \sum_{l=1}^{n_{h}} x_{\vartheta, n, l}(\tau)+D_{1, n}(\tau), y_{n}(\tau)=\sum_{\vartheta= \pm} \sum_{l=1}^{n_{h}} y_{\vartheta, n, l}(\tau)+D_{2, n}(\tau) .
$$

where $D_{n}$ is a small remainder satisfying

$$
\sup _{0 \leq \tau \leq \tau_{*}} \sup _{n}\left[\left|D_{1, n}(\tau)\right|+\left|D_{2, n}(\tau)\right|\right] \leq C_{\delta} \frac{\varrho}{\beta^{1+\delta}}
$$

with arbitrarily small positive $\delta$. Hence, the following statement holds.

Theorem 2.17 (superposition for Fermi-Pasta-Ulam equation) If every $\Phi_{l, \zeta}(\mathbf{r})$ is a Schwartz function, and the wavevectors $k_{* l} \neq 0$ satisfy 2.54), then the solution $x_{n}(\tau), y_{n}(\tau)$ of the initial value problem for the Fermi-Pasta-Ulam equation (2.10) with multi-wavepacket initial condition (2.53) is a linear superposition of solutions $x_{n, l}(\tau), y_{n, l}(\tau)$ of the same equation with single-wavepacket initial condition (2.56) up to a small coupling term $D_{1, n}(\tau), D_{2, n}(\tau)$ satisfying 2.57), (2.58) with arbitrary small $\delta>0$ and $\tau_{*}$ which do not depend on $\beta, \varrho, \delta$. 
Note that solutions $x_{\vartheta, n, l}(\tau)$ with different $\vartheta, l$ resemble $2 n_{h}$ solitons which originate at different points $r_{l}$ and propagate with different group velocities. According to (2.57), (2.58) all these soliton-like wavepackets pass through one another with very little interaction, see Fig. 1. Note that Theorem 2.15] shows that this phenomenon is robust in the class of general

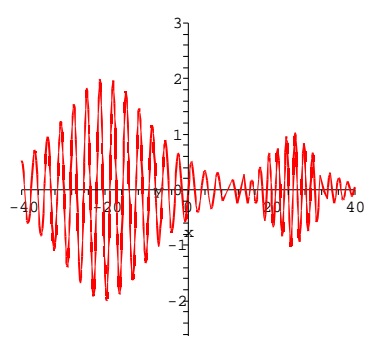

Figure 1: In this picture two wavepackets are shown with different "centers" $k_{* 1}$ and $k_{* 2}$. The values of $k_{* 1}$ and $k_{* 2}$ are proportional to the frequences of spatial oscillations. Though the wavepackets overlap in physical space, they pass one through another in the process of nonlinear evolution almost without interaction if their group velocities are different.

difference equations on the lattice $\mathbb{Z}$, and that it persists under polynomial perturbations of the nonlinearity as well as perturbations of the linear part of the equation (2.11) as long as they leave the linear difference operator non-positive and self-adjoint. Observe also that the evolution of every single wavepacket is nonlinear, and it is well-approximated by a properly constructed NLS (we intend to write a proof of this statement for general lattice systems in another article; see [23] for a particular case). For example, for a special choice of $\Psi_{j l}$ the solution $x_{n, l}(\tau)$ can be well approximated by a soliton solution of a corresponding NLS.

\subsection{Main statements and examples for semilinear systems of hy- perbolic PDE}

In this subsection we consider nonlinear evolution equation involving partial differential (and pseudodifferential) operators with respect to spatial variables with constant coefficients in the entire space $\mathbb{R}^{d}$. There is a great deal of similarity between such nonlinear evolution PDE and the lattice nonlinear evolution equations considered in the previous section. In particular, we study first not the original PDE but its Fourier transform, modal evolution equation, and the results concerning the original PDE are obtained by applying the inverse Fourier transform.

Recall that for functions $\mathbf{U}(\mathbf{r})$ from $L_{1}\left(\mathbb{R}^{d}\right)$ the Fourier transform and its inverse are 
defined by the formulas

$$
\begin{gathered}
\hat{\mathbf{U}}(\mathbf{k})=\int_{\mathbb{R}^{d}} \mathbf{U}(\mathbf{r}) \mathrm{e}^{-\mathrm{i} \mathbf{r} \cdot \mathbf{k}} \mathrm{d} \mathbf{r}, \quad \text { where } \mathbf{k} \in \mathbb{R}^{d}, \\
\mathbf{U}(\mathbf{r})=\frac{1}{(2 \pi)^{d}} \int_{\mathbb{R}^{d}} \hat{\mathbf{U}}(\mathbf{k}) \mathrm{e}^{\mathrm{i} \mathbf{r} \cdot \mathbf{k}} \mathrm{d} \mathbf{r}, \text { where } \mathbf{r} \in \mathbb{R}^{d} .
\end{gathered}
$$

Similarly to (2.3) we introduce the following modal evolution equation

$$
\partial_{\tau} \hat{\mathbf{U}}(\mathbf{k}, \tau)=-\frac{\mathrm{i}}{\varrho} \mathbf{L}(\mathbf{k}) \hat{\mathbf{U}}(\mathbf{k}, \tau)+\hat{F}(\hat{\mathbf{U}})(\mathbf{k}, \tau), \hat{\mathbf{U}}(\mathbf{k}, 0)=\hat{\mathbf{h}}(\mathbf{k}), \mathbf{k} \in \mathbb{R}^{d}
$$

where (i) $\hat{\mathbf{U}}(\mathbf{k}, \tau)$ is a $2 J$-component vector-function of $\mathbf{k}, \tau$, (ii) $\mathbf{L}(\mathbf{k})$ is a $2 J \times 2 J$ matrix function of $\mathbf{k}$, and (iii) $\hat{F}(\hat{\mathbf{U}})$ is the nonlinearity. We assume that the $2 J \times 2 J$ matrix $\mathbf{L}(\mathbf{k})$, $\mathbf{k} \in \mathbb{R}^{d}$, has exactly $2 J$ eigenvectors $\mathbf{g}_{n, \zeta}(\mathbf{k})$ with corresponding $2 J$ real eigenvalues $\omega_{n, \zeta}(\mathbf{k})$ satisfying the relation (2.13), (2.14) $(2.15),(2.16),(2.17)$. We also assume the matrix $\mathbf{L}(\mathbf{k})$, $\mathbf{k} \in \mathbb{R}^{d}$, to satisfy the polynomial bound

$$
|\mathbf{L}(\mathbf{k})| \leq C\left(1+|\mathbf{k}|^{p}\right) .
$$

The singular set $\sigma$ for $\mathbf{L}(\mathbf{k})$ is as in Definition 2.3 with the only difference that functions $\omega_{n, \zeta}(\mathbf{k})$ are defined over $\mathbb{R}^{d}$ rather than the torus $[-\pi, \pi]^{d}$, and, consequently they are not periodic. The nonlinearity $\hat{F}(\hat{\mathbf{U}})$ has a form entirely similar to (2.21):

$$
\hat{F}(\hat{\mathbf{U}})=\sum_{m=2}^{m_{F}} \hat{F}^{(m)}\left(\hat{\mathbf{U}}^{m}\right),
$$

with $\hat{F}^{(m)}$ being $m$-linear operators with the following representation similar to (2.22):

$$
\hat{F}^{(m)}\left(\hat{\mathbf{U}}_{1}, \ldots, \hat{\mathbf{U}}_{m}\right)(\mathbf{k})=\int_{\mathbb{D}_{m}} \chi^{(m)}(\mathbf{k}, \vec{k}) \hat{\mathbf{U}}_{1}\left(\mathbf{k}^{\prime}\right) \ldots \hat{\mathbf{U}}_{m}\left(\mathbf{k}^{(m)}(\mathbf{k}, \vec{k})\right) \tilde{\mathrm{d}}^{(m-1) d} \vec{k}
$$

where $\mathbf{k}^{(m)}(\mathbf{k}, \vec{k})$ is defined by the convolution equation (2.25), $\tilde{\mathrm{d}}$ is defined by (2.24) and $\mathbb{D}_{m}$ in (2.64) is now defined not by (2.23) but by

$$
\mathbb{D}_{m}=\mathbb{R}^{(m-1) d} \text {. }
$$

The difference with (2.3) now is that the involved functions of $\mathbf{k}, \mathbf{k}^{\prime}$ etc., are not $2 \pi$-periodic, $\mathbb{D}_{m}$ in (2.64) is defined by (2.65) instead of (2.23) , and the tensors $\chi^{(m)}(\mathbf{k}, \vec{k})$ satisfy the nonlinear regularity Condition 2.6 without the periodicity assumption. The functions $\hat{\mathbf{U}}_{l}\left(\mathbf{k}^{(l)}\right)$ in (2.64) are assumed to be from the space $L_{1}=L_{1}\left(\mathbb{R}^{d}\right)$ with the norm

$$
\|\hat{\mathbf{U}}(\cdot)\|_{L_{1}}=\int_{\mathbb{R}^{d}}|\tilde{\mathbf{v}}(\mathbf{k})| \mathrm{d} \mathbf{k} .
$$


We seek solutions to (2.61) in the space $C^{1}\left(\left[0, \tau_{*}\right], L_{1}\right)$ with $0<\tau_{*} \leq 1$.

Applying the inverse Fourier transform to the modal evolution equation (2.61) we obtain a hyperbolic $2 J$-component systems in $\mathbb{R}^{d}$ of the form

$$
\partial_{\tau} \mathbf{U}(\mathbf{r}, \tau)=-\frac{\mathrm{i}}{\varrho} \mathbf{L}\left(-\mathrm{i} \nabla_{\mathbf{r}}\right) \mathbf{U}(\mathbf{r}, \tau)+F(\mathbf{U})(\mathbf{r}, \tau), \mathbf{U}(\mathbf{r}, 0)=\mathbf{h}(\mathbf{r}) .
$$

Note that since $\mathbf{L}(\mathbf{k})$ satisfies the polynomial bound 2.62) we can define the action of the operator $\mathbf{L}\left(-\mathrm{i} \nabla_{\mathbf{r}}\right)$ on any Schwartz function $\mathbf{Y}(\mathbf{r})$ by the formula

$$
\mathbf{L}\left(\widehat{-\mathrm{i} \nabla_{\mathbf{r}}}\right) \mathbf{Y}(\mathbf{k})=\mathbf{L}(\mathbf{k}) \hat{\mathbf{Y}}(\mathbf{k})
$$

where, in view of (2.62), the order of $\mathbf{L}$ does not exceed $p$. If all the entries of $\mathbf{L}(\mathbf{k})$ are polynomials, such a definition coincides with the common definition of the action of a differential operator $\mathbf{L}\left(-\mathrm{i} \nabla_{\mathbf{r}}\right)$. In this case $\mathbf{L}\left(-\mathrm{i} \nabla_{\mathbf{r}}\right)$ defined by (2.68) is a differential operator with constant coefficients of order not greater than $p$.

The properties of the modal evolution equation (2.61) are completely similar to its lattice counterpart and are as follows. The existence and uniqueness theorem is similar to Theorem 2.8 .

Theorem 2.18 (existence and uniqueness) Let equation (2.61) satisfy conditions 2.17) and (2.26) and $\mathbf{h} \in L_{1}=L_{1}\left(\mathbb{R}^{d}\right),\|\tilde{\mathbf{h}}\|_{L_{1}} \leq R$. Then there exists a unique solution to the modal evolution equation (2.61) in the functional space $C^{1}\left(\left[0, \tau_{*}\right], L_{1}\right)$. The number $\tau_{*}$ depends on $R, C_{\chi}$ and $C_{\Xi}$.

Here is the main result for the semilinear hyperbolic systems of PDE which is completely similar to Theorem 2.15.

Theorem 2.19 (principle of superposition for PDE systems) Let the initial data of the modal evolution equation (2.61) be a multi-wavepacket, i.e. the sum of $N_{h}$ wavepackets $\hat{\mathbf{h}}_{l}$ as in (2.45) satisfying Definitions 2.9. 2.10. Suppose that $\varrho, \beta$ satisfy condition (2.46). Assume also that $\hat{\mathbf{h}}$ is generic in the sense of Definition 2.14. Then the solution $\hat{\mathbf{U}}=\mathcal{G}(\hat{\mathbf{h}})$ to the modal evolution equation (2.61) satisfies the approximate linear superposition principle, namely

$$
\mathcal{G}\left(\sum_{l=1}^{N_{h}} \hat{\mathbf{h}}_{l}\right)=\sum_{l=1}^{N_{h}} \mathcal{G}\left(\hat{\mathbf{h}}_{l}\right)+\hat{\mathbf{D}}
$$

with a small remainder $\hat{\mathbf{D}}(\tau)$

$$
\sup _{0 \leq \tau \leq \tau_{*}}\|\hat{\mathbf{D}}(\tau)\|_{L_{1}} \leq C_{\epsilon} \frac{\varrho}{\beta^{1+\epsilon}}|\ln \beta|
$$


where $\epsilon$ is the same as in Definition [2.9, $\tau_{*}$ does not depend on $\beta, \varrho$ and $\epsilon$. The solutions $\mathbf{U}(\mathbf{h})(\mathbf{r}, \tau)$ of the space evolution equation (2.67) are obtained as the inverse Fourier transform of $\mathcal{G}(\hat{\mathbf{h}})$ and they satisfy the approximate linear superposition principle, namely

$$
\mathbf{U}(\mathbf{h})=\mathbf{U}\left(\mathbf{h}_{1}\right)+\ldots+\mathbf{U}\left(\mathbf{h}_{N_{h}}\right)+\mathbf{D}
$$

with a small coupling remainder $\mathbf{D}(\tau)$ satisfying

$$
\sup _{0 \leq \tau \leq \tau_{*}}\|\mathbf{D}(\tau)\|_{L_{\infty}} \leq C_{\epsilon} \frac{\varrho}{\beta^{1+\epsilon}}|\ln \beta|
$$

where $\epsilon>0$ is the same as in Definition 2.9 and can be arbitrary small.

Example 1: Sine-Gordon and Klein-Gordon equations with small initial data Let us consider the sine-Gordon equation (see [26])

$$
\partial_{t}^{2} u=\partial_{r}^{2} u-\sin u
$$

with small initial data

$$
u(r, 0)=\beta b_{0}, \partial_{t} u(r, 0)=\beta b_{1}, \beta \ll 1
$$

First, we recast this the equation into our framework by rescaling the variables

$$
u=\beta U_{1}, \beta^{2} t=\tau .
$$

Since $\sin \beta U_{1}=\beta U_{1}-\frac{1}{6} \beta^{3} U_{1}^{3}+\beta^{5} f\left(U_{1}\right)$, where evidently $f\left(U_{1}\right)$ is an enitire function, we can recast the equation (2.73) into the following form

$$
\partial_{\tau}^{2} U_{1}=\frac{1}{\beta^{4}}\left[\partial_{x}^{2} U_{1}-U_{1}\right]+\frac{1}{\beta^{2}}\left[q U_{1}^{3}+\beta^{2} f\left(U_{1}\right)\right]
$$

We introduce then a linear pseudodifferential operator $A=\left(I-\partial_{x}^{2}\right)^{1 / 2}$ with the symbol $\left(1+k^{2}\right)^{1 / 2}$ and rewrite the equation (2.76) as the following system

$$
\partial_{\tau} U_{1}=\frac{1}{\beta^{2}} A U_{2}, \partial_{\tau} U_{2}=-\frac{1}{\beta^{2}} A U_{1}+A^{-1}\left[q U_{1}^{3}+\beta^{2} f\left(U_{1}\right)\right],
$$

with the initial data

$$
U_{1}(0)=h_{0}, U_{2}(0)=h_{1},
$$

where $h_{0}$ and $h_{1}$ are assumed to be of the form

$$
z(\mathbf{r}, 0)=h_{0}, p(\mathbf{r}, 0)=h_{1}, h_{j}=\sum_{l=1}^{n_{h}} \Psi_{j l}\left(\beta \mathbf{r}-\mathbf{r}_{l}\right) \mathrm{e}^{\mathrm{i} \mathbf{k}_{* l} \cdot \mathbf{r}}+c c, j=0,1,
$$


in one-dimentional case with $\mathbf{r}=r, \mathbf{k}=k$. Evidently, the relations with the initial data of (2.73) are

$$
b_{0}=h_{0}, b_{1}=A h_{1} .
$$

Notice that the system (2.77) is of the form (2.67) with

$$
\begin{gathered}
\varrho=\beta^{2}, \mathbf{L} \mathbf{U}=\left[\begin{array}{c}
A U_{2} \\
-A U_{1}
\end{array}\right], F(\mathbf{U})=F_{0}(\mathbf{U})+\beta^{2} F_{1}(\mathbf{U}), \\
F_{0}(\mathbf{U})=A^{-1}\left[\begin{array}{c}
0 \\
q U_{1}^{3}
\end{array}\right], F_{1}(\mathbf{U})=A^{-1}\left[\begin{array}{c}
0 \\
f\left(U_{1}\right)
\end{array}\right] .
\end{gathered}
$$

Observe now that $\mathbf{L}$ has only one spectral band with the dispersion relation and eigenvectors given by

$$
\omega(k)=\left(I+k^{2}\right)^{1 / 2}, \mathbf{g}_{\vartheta}(k)=\mathbf{g}_{\vartheta}=2^{-1 / 2}\left[\begin{array}{c}
-\mathrm{i} \vartheta \\
1
\end{array}\right], \quad \vartheta= \pm 1,
$$

and there is no band-crossing points. We use expansion in the basis $\mathbf{g}_{ \pm}$

$$
\left[\begin{array}{c}
\Psi_{0 l} \\
\Psi_{1 l}
\end{array}\right] \mathrm{e}^{\mathrm{i} \mathbf{k}_{* l} \cdot \mathbf{r}}=\left[\Phi_{+, l} \mathbf{g}_{+}+\Phi_{-, l} \mathbf{g}_{-}\right] \mathrm{e}^{\mathrm{i} \mathbf{k}_{* l} \cdot \mathbf{r}} .
$$

to represent initial data (2.78) and (2.79). The equation (2.42) takes here the form

$$
\left(1+k_{* l}^{2}\right)^{1 / 2} \lambda=\zeta\left(1+\lambda^{2} k_{* l}^{2}\right)^{1 / 2}, \zeta= \pm 1 .
$$

Obviously, this equation has only solutions $\lambda=\zeta$ and Condition 2.13 is fulfilled. Condition 2.12 holds if

$$
\frac{\vartheta k_{* l}}{\left(1+k_{* l}^{2}\right)^{1 / 2}} \neq \frac{\vartheta^{\prime} k_{* l^{\prime}}}{\left(1+k_{* l^{\prime}}^{2}\right)^{1 / 2}} \text { for } l \neq l^{\prime} \text { or } \vartheta \neq \vartheta^{\prime}
$$

which is equivalent to

$$
k_{* l^{\prime}} \neq k_{* l} \text { for } l^{\prime} \neq l \text {, and } k_{* l} \neq 0 \text { for all } l \text {. }
$$

Equation (2.77) can be written in the integral form (3.3) with $m_{F}=\infty$ and by Theorem 5.4 it has unique solution $\mathbf{U}$ for $\tau \leq \tau_{*}$. If we replace $F(\mathbf{U})$ in (2.80) by $F_{0}(\mathbf{U})$ we obtain

$$
\partial_{\tau} V_{1}=\frac{1}{\beta^{2}} A V_{2}, \partial_{\tau} V_{2}=-\frac{1}{\beta^{2}} A V_{1}+A^{-1} q V_{1}^{3}
$$

where we take the initial data to be as in (2.78), namely

$$
V_{1}(0)=h_{0}, V_{2}(0)=h_{1} .
$$

Equations (2.84) can be obtained by replacing $\sin u$ in (2.73) by the cubic polynomial $u-u^{3} / 6$ producing the quasilinear Klein-Gordon equation (see 36]). Observe that the solutions to the sine-Gordon and the Klein-Gordon equations with small initial data are very close. To 
see that, note that the operator $\widehat{f(U)}(k)$ is bounded in $L_{1}$ for $\widehat{U}(k)$ which are bounded in $L_{1}$. Therefore the norm of the neglected term is small, namely $\left\|\beta^{2} \widehat{f(U)}\right\|_{L_{1}} \leq C \beta^{2}$. Thus, by Remark 4.8, the solutions of (2.77) and (2.84) are close, namely

$$
\left\|U_{1}-V_{1}\right\|_{L_{\infty}}+\left\|U_{2}-V_{2}\right\|_{L_{\infty}} \leq C \beta^{2}, 0 \leq \tau \leq \tau_{*}
$$

According to Theorem 2.19] the superposition principle is applicable to the equation (2.84) with initial data as in (2.85), and the following statements hold.

Theorem 2.20 (Superposition for Klein-Gordon) Assume that the initial data $h_{0}, h_{1}$ in (2.85) are as in (2.79). Then the solution $\left\{V_{1}, V_{2}\right\}$ to the system 2.84) satisfies the linear superposition principle, namely

$$
V_{1}(\mathbf{r}, \tau)=\sum_{\vartheta= \pm} \sum_{l=1}^{n_{h}} V_{1, \vartheta, l}(\mathbf{r}, \tau)+\mathbf{D}_{1}(\mathbf{r}, \tau), V_{2}(\mathbf{r}, \tau)=\sum_{\vartheta= \pm} \sum_{l=1}^{n_{h}} V_{2, \vartheta, l}(\mathbf{r}, \tau)+\mathbf{D}_{2}(\mathbf{r}, \tau)
$$

where $\left\{V_{1, \vartheta, l}(\mathbf{r}, \tau), V_{2, \vartheta, l}(\mathbf{r}, \tau)\right\}$ is a solution to (2.84) with the one-wavepacket initial condition

$$
\left[\begin{array}{l}
V_{1, \vartheta, l}(\mathbf{r}, 0) \\
V_{2, \vartheta, l}(\mathbf{r}, 0)
\end{array}\right]=\Phi_{\vartheta, l}\left(\beta \mathbf{r}-\mathbf{r}_{l}\right) \mathbf{g}_{\vartheta} \mathrm{e}^{\mathrm{i} \mathbf{k}_{* l} \cdot \mathbf{r}}+\mathrm{cc},
$$

where $\Phi_{\vartheta, l}(\mathbf{r})$ are arbitrary Schwartz functions. If (2.83) holds, the coupling terms $\mathbf{D}_{1}, \mathbf{D}_{2}$ satisfy the bound

$$
\sup _{0 \leq \tau \leq \tau_{*}}\left[\left\|\mathbf{D}_{1}(\tau)\right\|_{L_{\infty}}+\left\|\mathbf{D}_{2}(\tau)\right\|_{L_{\infty}}\right] \leq C_{\delta}^{\prime} \frac{\varrho}{\beta^{1+\delta}}=C_{\delta}^{\prime} \beta^{1-\delta}
$$

where $\tau_{*}$ and $C_{\delta}^{\prime}$ do not depend on $\beta$, and $\delta$ can be taken arbitrary small.

Using (2.86) we obtain a similar superposition theorem for the sine-Gordon equation.

Theorem 2.21 (Superposition for sine-Gordon) Assume that the initial data $h_{0}, h_{1}$ in (2.78) are as in (2.79). Then the solution $\left\{U_{1}, U_{2}\right\}$ to (2.77), 2.78) satisfies the linear superposition principle, namely

$$
U_{1}(\mathbf{r}, \tau)=\sum_{\vartheta= \pm} \sum_{l=1}^{n_{h}} U_{1, \vartheta, l}(\mathbf{r}, \tau)+\mathbf{D}_{1}(\mathbf{r}, \tau), U_{2}(\mathbf{r}, \tau)=\sum_{\vartheta= \pm} \sum_{l=1}^{n_{h}} U_{2, \vartheta, l}(\mathbf{r}, \tau)+\mathbf{D}_{2}(\mathbf{r}, \tau)
$$

where $U_{1, \vartheta, l}(\mathbf{r}, \tau), U_{2, \vartheta, l}(\mathbf{r}, \tau)$ is a solution of 2.77) with the one-wavepacket initial condition

$$
\left[\begin{array}{c}
U_{1, \vartheta, l}(\mathbf{r}, 0) \\
U_{2, \vartheta, l}(\mathbf{r}, 0)
\end{array}\right]=\Phi_{\vartheta, l}\left(\beta \mathbf{r}-\mathbf{r}_{l}\right) \mathbf{g}_{\vartheta} \mathrm{e}^{\mathrm{i} \mathbf{k}_{* l} \cdot \mathbf{r}}+\mathrm{cc}, \vartheta= \pm
$$

where $\Phi_{\vartheta, l}(\mathbf{r})$ are arbitrary Schwartz functions. If (2.83) holds, the coupling terms $\mathbf{D}_{1}, \mathbf{D}_{2}$ satisfy the bound (2.89). 
Note that a theorem completely similar to Theorem 2.20 holds also for a generalized Klein-Gordon equation where $q V_{1}^{3}$ is replaced by an arbitrary polynomial $P\left(V_{1}\right)$. Hence, the superposition principle holds for the sine-Gordon equation (2.73) with a small initial data and a strongly perturbed nonlinearity as, for example, when $\sin u$ is replaced by $\sin u+\beta^{-1} u^{4}+$ $\beta^{-2} u^{5}$.

We would like to compare now our results and methods with that of [38] where the interaction of counterpropagating waves is studied by the ansatz method. Pierce and Wayne considered in 38 the sine-Gordon equation in the case of small initial data which have the form of a bimodal wavepacket. In our notation it corresponds to the case when $\varrho=\beta^{2}$, $n_{h}=1$ in (2.79), when two wavepackets, corresponding to $\vartheta=+$ and $\vartheta=-$, have exactly opposite group velocities. They proved that the bimodal wavepacket data generate two waves which are described by two uncoupled nonlinear Schrodinger equations with a small error. The magnitude of the error given in 38. (which we formulate here for the solution $U_{1}$ of the rescaled equation (2.76) $)$ is estimated by $C \beta^{1 / 2}$ on the time interval $0 \leq \tau \leq \tau_{0}$ ( or $0 \leq t \leq \tau_{0} \beta^{-2}$ ). Note that our general Theorem 2.19] when applied to the special case of the sine-Gordon equation (2.76) provides a better estimate of the coupling error, namely $C \varrho / \beta^{1+\delta}=C \beta^{1-\delta}$ in (2.89) with arbitrary small $\delta$, for the same time interval. Notice that the estimate (2.72) given in Theorem 2.19] is almost optimal, since it is possible to construct examples when the coupling error is greater than $c \beta^{1+\delta}$ with arbitrary small $\delta$.

We would like to point out that the general mechanism responsible for the wavepacket decoupling is the destructive wave interference, this mechanism is subtle though general. We treat the destructive wave interference by taking into account explicitly all nonlinear interactions of high-frequency waves. In our approach we use the exact representation of a general solution in the form of a functional-analytic operator monomial series, every term of the series is explicitly given as a multilinear oscillatory integral operator applied to the initial data. A key advantage of such an approach is that it allows to estimate wavepacket coupling as a sum of contributions of highly oscillatory terms and to get a precise estimate of magnitude of every term. In contrast, the well known "ansatz" approach as, for instance, in 38 and 32, requires to find a clever ansatz with consequent estimations of the "residuum" in an appropriate norm. Our approach can naturally treat general tensorial polynomial nonlinearities $F$ of arbitrary large degree $N_{F}$ and any number of wavepackets, whereas finding a good ansatz which allows to estimate the residuum in such a general situation would be difficult. For readers interested in detailed features of one-wavepacket solutions to the sineGordon equations, we refer to 32, 38] and 39].

Example 2: Nonlinear Schrodinger equation. The Nonlinear Schrodinger equation (NLS) with $d$ spatial variables ([42, [16], [15]) has the form

$$
\partial_{\tau} z(\mathbf{r}, \tau)=\mathrm{i} \frac{1}{\varrho} \gamma(-\mathrm{i} \nabla) z(\mathbf{r}, \tau)+\alpha|z|^{2} z(\mathbf{r}, \tau), z(\mathbf{r}, 0)=h(\mathbf{r}), \mathbf{r} \in \mathbb{R}^{d},
$$


where $\alpha$ is a complex constant, $\gamma(-\mathrm{i} \nabla)$ is a second-order differential operator, its symbol $\gamma(\mathbf{k})$ is a real, symmetric quadratic form

$$
\gamma(\mathbf{k})=\gamma(\mathbf{k}, \mathbf{k})=\sum \gamma_{i j} k_{i} k_{j}, \gamma(-\mathrm{i} \nabla) z=-\sum \gamma_{i j} \partial_{r_{i}} \partial_{r_{j}} z .
$$

To put the NLS into the framework of this paper we introduce the following two-component system

$$
\begin{aligned}
\partial_{\tau} z_{+}(\mathbf{r}, \tau) & =\mathrm{i} \frac{1}{\varrho} \gamma(-\mathrm{i} \nabla) z_{+}(\mathbf{r}, \tau)+\alpha z_{-} z_{+}^{2}(\mathbf{r}, \tau), \\
\partial_{\tau} z_{-}(\mathbf{r}, \tau) & =-\mathrm{i} \frac{1}{\varrho} \gamma(\mathrm{i} \nabla) z_{-}(\mathbf{r}, \tau)+\alpha^{*} z_{+} z_{-}^{2}(\mathbf{r}, \tau), \\
z_{+}(\mathbf{r}, 0) & =h(\mathbf{r}), z_{-}(\mathbf{r}, 0)=h^{*}(\mathbf{r}), \mathbf{r} \in \mathbb{R}^{d},
\end{aligned}
$$

where $\alpha^{*}$ denotes complex conjugate to $\alpha$. Obviously if $z(\mathbf{r}, \tau)$ is a solution of (2.90) then $z_{+}(\mathbf{r}, \tau)=z(\mathbf{r}, \tau), z_{-}(\mathbf{r}, \tau)=z^{*}(\mathbf{r}, \tau)$ gives a solution of (2.91). Using the Fourier transform we get from (2.90)

$$
\partial_{\tau} \hat{z}(\mathbf{k}, \tau)=\mathrm{i} \frac{1}{\varrho} \gamma(\mathbf{k}) \hat{z}(\mathbf{k}, \tau)+\alpha \widehat{\left(z^{*} z^{2}\right)}(\mathbf{k}, \tau), \mathbf{k} \in \mathbb{R}^{d} .
$$

Now the band-crossing set $\sigma=\left\{\mathbf{k} \in \mathbb{R}^{d}: \gamma(\mathbf{k})=0\right\}$. We assume that the quadratic form $\gamma$ is not identically zero. The Fourier transform of (2.91) takes the form of (2.67) with

$$
\begin{gathered}
\hat{\mathbf{U}}=\left[\begin{array}{c}
\hat{U}_{+} \\
\hat{U}_{-}
\end{array}\right], \mathbf{L}(\mathbf{k}) \hat{\mathbf{U}}=\left[\begin{array}{cc}
\gamma(\mathbf{k}) & 0 \\
0 & -\gamma(-\mathbf{k})
\end{array}\right]\left[\begin{array}{l}
\hat{U}_{+} \\
\hat{U}_{-}
\end{array}\right], \\
\omega(\mathbf{k})=|\gamma(\mathbf{k})|, \hat{F}^{(3)}\left(\hat{\mathbf{U}}^{3}\right)=\left[\begin{array}{c}
\alpha\left(\hat{z}_{+}(\hat{\mathbf{U}}) \hat{z_{+}(\hat{\mathbf{U}})} \hat{z}_{-}(\hat{\mathbf{U}})\right) \\
\alpha^{*}\left(\hat{z}_{-}(\hat{\mathbf{U}}) \hat{z_{-}(\hat{\mathbf{U}})} \hat{z}_{+}(\hat{\mathbf{U}})\right)
\end{array}\right],
\end{gathered}
$$

To satisfy the requirements of Condition 2.14 we have to take the wave vectors $\mathbf{k}_{* l} \notin \sigma$ so that

$$
\nabla\left|\gamma\left(\mathbf{k}_{* l}\right)\right|=\frac{2 \gamma\left(\mathbf{k}_{* l}\right)}{\left|\gamma\left(\mathbf{k}_{* l}\right)\right|} \gamma\left(\mathbf{k}_{* l}, \cdot\right) \neq \frac{2 \gamma\left(\mathbf{k}_{* l^{\prime}}\right)}{\left|\gamma\left(\mathbf{k}_{* l^{\prime}}\right)\right|} \gamma\left(\mathbf{k}_{* l^{\prime}}, \cdot\right) \text { if } l \neq l^{\prime},
$$

which provides (2.41). Since

$$
\left|\gamma\left(\mathbf{k}_{* l}\right)\right| \lambda-\zeta\left|\gamma\left(\lambda \mathbf{k}_{* l}\right)\right|=\left|\gamma\left(\mathbf{k}_{* l}\right)\right|\left[\lambda-\zeta|\lambda|^{2}\right],
$$

and $\lambda$ is odd, every point $\mathbf{k}_{* l} \notin \sigma$ satisfies Condition 2.13. If the quadratic form $\gamma$ is not singular, that is $\operatorname{det} \gamma \neq 0$, then condition (2.93), which ensures that group velocities of wavepackets are different, holds when

$$
\frac{\gamma\left(\mathbf{k}_{* l}\right)}{\left|\gamma\left(\mathbf{k}_{* l}\right)\right|} \mathbf{k}_{* l} \neq \frac{\gamma\left(\mathbf{k}_{* l^{\prime}}\right)}{\left|\gamma\left(\mathbf{k}_{* l^{\prime}}\right)\right|} \mathbf{k}_{* l^{\prime}} \text { if } l \neq l^{\prime}
$$

In this case Theorem 2.19 is applicable, and generic wavepacket solutions of the NLS are linearly superposed and propagate almost independently with coupling $O(\beta)$. More precisely, as a corollary of Theorem 2.19] we obtain the following statement. 
Theorem 2.22 (Superposition for NLS) Assume that initial data of the NLS (2.90) have the form $h=h_{1}+\ldots+h_{N_{h}}$

$$
h_{l}(\mathbf{r})=\mathrm{e}^{\mathrm{i} \mathbf{k}_{* l} \cdot \mathbf{m}} \Phi_{l,+}\left(\beta \mathbf{r}-\mathbf{r}_{0}\right)+\mathrm{e}^{-\mathrm{i} \mathbf{k}_{* l} \cdot \mathbf{m}} \Phi_{l,-}\left(\beta \mathbf{r}-\mathbf{r}_{0}\right), l=1, \ldots, N_{h}
$$

where $\Phi_{l, \zeta}(\mathbf{r})$ are arbitrary Schwartz functions. Assume also that $\operatorname{det} \gamma \neq 0$ and the vectors $\mathbf{k}_{* l}$ satisfy conditions

$$
\gamma\left(\mathbf{k}_{* l}\right) \neq 0, l=1, \ldots, N_{h} ; \mathbf{k}_{* l} \neq \mathbf{k}_{* l^{\prime}} \text { if } l \neq l^{\prime} .
$$

Then solution $z=z(h)$ is a linear superposition

$$
z(h)=z\left(h_{1}\right)+\ldots+z\left(h_{N_{h}}\right)+D
$$

with a small coupling term $D$

$$
\sup _{0 \leq \tau \leq \tau_{*}}\|D(\tau)\|_{L_{\infty}\left(\mathbb{R}^{d}\right)} \leq C_{\delta} \frac{\varrho}{\beta^{1+\delta}},
$$

where $\delta>0$ can be taken arbitrary small.

We note in conclusion, that the superposition principle reduces dynamics of multi-wavepacket solutions to dynamics of single-wavepacket solutions; we do not study dynamics of singlewavepacket solutions in this paper. Note that the theory of NLS-type approximations of one-wavepacket solutions of hyperbolic PDE is well-developed, see [29, [30], [18, [40, 41], [5] and references therein. Relevance of different group velocities of wavepackets for smallness of their interaction was noted in [29].

\subsection{Generalizations}

Note that in a degenerate case when the function $\omega_{n_{l}}(\mathbf{k})$ is linear in the direction of $\mathbf{k}_{*}$ the equation (2.42) for $\zeta=1$ has many solutions for which $\theta \neq \pm 1$ and Condition 2.13 does not hold. It turns out, that if Condition 2.13 for dispersion relations $\omega_{n}(\mathbf{k})$ at $\mathbf{k}_{*}$ is not satisfied, still we can prove our results under the following alternative condition. We consider here the case of PDE in the entire space $\mathbb{R}^{d}$ and $\mathbf{k} \in \mathbb{R}^{d}$.

Condition 2.23 (complete degeneracy) The series (2.21) has only $\tilde{F}^{(m)}$ with odd $m$. The wavevectors $\mathbf{k}_{* l}$ and functions $\omega_{n_{l}}(\mathbf{k}), l=1, \ldots, N_{h}$, have the following three properties:

(i) There exists $\delta>0$ such that for every $l_{1} \neq l_{2}$, the following inequality holds:

$$
\left|\nabla_{\mathbf{k}} \omega_{n_{l_{1}}}\left(\nu_{1} \mathbf{k}_{* l_{1}}\right)-\nabla_{\mathbf{k}} \omega_{n_{l_{2}}}\left(\nu_{2} \mathbf{k}_{* l_{2}}\right)\right| \geq \delta,
$$

for any odd integers $\nu_{1}, \nu_{2}=1,3, \ldots$.

(ii) There exists $\delta>0$ such that $\nu \mathbf{k}_{* l}$ does not get in a $\delta$-neighborhood of $\sigma$ for any odd integer $\nu$ and any $l=1, \ldots, N_{h}$. 
(iii) For any positive integer odd number $\theta$ and any $\mathbf{k}_{* l}$, for any $n$ the following identities hold:

$$
\begin{aligned}
\nabla_{\mathbf{k}} \omega_{n}\left(\theta \mathbf{k}_{* l}\right) & =\nabla_{\mathbf{k}} \omega_{n}\left(\mathbf{k}_{* l}\right), \\
\omega_{n}\left(\theta \mathbf{k}_{* l}\right) & =\theta \omega_{n}\left(\mathbf{k}_{* l}\right) .
\end{aligned}
$$

A nontrivial examples, where the above Condition 2.23 is satisfied, is given below.

We give here a generalization of Definition 2.14.

Definition 2.24 (generic multi-wavepackets) A multi-wavepacket $\hat{\mathbf{h}}$ as defined in Definition 2.10 is called generic if $(i)$ the centers $\mathbf{k}_{* l}, l=1, \ldots, N_{h}$, of all wavepackets satisfy Conditions [2.11] and 2.12; (ii) either the dispersion relations $\omega_{n}(\mathbf{k})$ at every $\mathbf{k}_{* l}$ and band $n_{l}$ satisfy Condition 2.13 or they satisfy Condition 2.23.

The statement of Theorem 2.19 remains true if Condition 2.14 is replaced by less restrictive Condition 2.24, namely the following theorem holds.

Theorem 2.25 Let the initial data of the modal evolution equation (2.61) be a multi-wavepacket, i.e. the sum of $N_{h}$ wavepackets $\hat{\mathbf{h}}_{l}$ as in (2.45) satisfying Definitions 2.9, 2.10. Suppose that (2.46) holds. Assume also that $\hat{\mathbf{h}}$ is generic in the sense of Definition 2.24. Then the solution $\hat{\mathbf{U}}=\mathcal{G}(\hat{\mathbf{h}})$ to the modal evolution equation (2.61) satisfies the approximate linear superposition principle, namely (2.69), 2.70), 2.71) and (2.72) hold.

The proofs we give in this paper directly apply to more general Theorem 2.25 .

Another generalization concerns the possibility to shift independently initial wavepackets. If initial data involve parameters $\mathbf{r}_{l}$ as in (2.79) it is possible to prove that $C_{\epsilon}$ in (2.48), (2.70) and (2.72) does not depend on $\mathbf{r}_{l} \in \mathbb{R}^{d}$ if the functions $\Psi_{j l}$ are Schwartz functions. Most of the proofs remain the same, but several statements have to be modified, and we present proofs in a subsequent paper.

One more generalization concerns the smoothness of initial data. It is possible to take initial data $\mathbf{h}_{l}(\mathbf{r})$ with a finite smoothness rather than from Schwartz class. Namely, consider weighted spaces $L_{1, a}$ with the norm

$$
\|\hat{\mathbf{v}}\|_{L_{1, a}}=\int_{\mathbb{R}^{d}}(1+|\mathbf{k}|)^{a}|\hat{\mathbf{v}}(\mathbf{k})| \mathrm{d} \mathbf{k}, a \geq 0 .
$$

Obviously, large $a$ corresponds to high smoothness of the inverse Fourier transform $\mathbf{v}(\mathbf{r})$. Then if functions $\hat{\mathbf{h}}_{l, \zeta}(\mathbf{k})$ have the form (2.36) with $\hat{h}_{\zeta}(\mathbf{k})=\hat{h}_{l, \zeta}(\mathbf{k})$ from the class $L_{1, a}$ the inequality (2.70) can be replaced by

$$
\sup _{0 \leq \tau \leq \tau_{*}}\|\hat{\mathbf{D}}(\tau)\|_{L_{1}} \leq C_{\epsilon} \frac{\varrho}{\beta^{1+\epsilon}}|\ln \beta|+C_{\epsilon} \beta^{s}
$$


where $s>0$ and $\epsilon>0$ have to satisfy restriction $\frac{s}{\epsilon}<a$. This generalization requires minor modifications in the proofs and in conditions (2.33) and (2.34) $C_{\epsilon} \beta$ has to be replaced by $C_{\epsilon} \beta^{s}$. In particular, if $a=1, \varrho=\beta^{2}$ and $s=1 / 2$ the right-hand side of (2.98) can be estimated by $C_{\epsilon_{1}} \beta^{1 / 2-\epsilon_{1}}$ with arbitrary small $\epsilon_{1}$.

More generalizations which involve the structure of equations are discussed in Sections 7.3 and 7.4. Now we give an example where Condition 2.23 is applicable.

Example 3: Semilinear wave equation. Let us consider a semilinear wave equation with $d$ spatial variables

$$
\partial_{\tau}^{2} z(\mathbf{r}, \tau)=\frac{1}{\varrho^{2}} \Delta z(\mathbf{r}, \tau)+\frac{\alpha}{\varrho} \partial_{x_{1}} z^{3}(\mathbf{r}, \tau), \mathbf{r} \in \mathbb{R}^{d},
$$

where $\Delta$ is the Laplace operator, $\alpha$ is an arbitrary complex constant, $\varrho=\beta^{2}$. We introduce the operator $A=\sqrt{-\Delta}$ which is defined in terms of the Fourier transform, it has symbol $|\mathbf{k}|$. We rewrite (2.99) in the form of a first-order system

$$
\begin{gathered}
\partial_{\tau} z(\mathbf{r}, \tau)=\frac{1}{\varrho} A p(\mathbf{r}, \tau), \mathbf{r} \in \mathbb{R}^{d} \\
\partial_{\tau} p(\mathbf{r}, \tau)=-\frac{1}{\varrho} A z(\mathbf{r}, \tau)+\alpha A^{-1} \partial_{x_{1}} z^{3}(\mathbf{r}, \tau) .
\end{gathered}
$$

The linear operator $A^{-1} \partial_{x_{1}}$ has the symbol $\frac{-\mathrm{i} k_{1}}{|\mathbf{k}|}$, it is a zero order operator. We rewrite (2.100) in the form of (2.67) where

$$
\mathbf{U}=\left[\begin{array}{c}
z \\
p
\end{array}\right],-\mathrm{i} \mathbf{L}\left(-\mathrm{i} \nabla_{\mathbf{r}}\right) \mathbf{U}=\left[\begin{array}{cc}
0 & A \\
-A & 0
\end{array}\right]\left[\begin{array}{c}
z \\
p
\end{array}\right], F\left(\left[\begin{array}{c}
z \\
p
\end{array}\right]\right)=\alpha\left[\begin{array}{c}
0 \\
-A^{-1} \partial_{x_{1}} z^{3}
\end{array}\right] .
$$

Using the Fourier transform we get (2.61) with

$$
\begin{gathered}
\hat{\mathbf{U}}=\left[\begin{array}{c}
\hat{z} \\
\hat{p}
\end{array}\right],-\mathrm{i} \mathbf{L}(\mathbf{k}) \hat{\mathbf{U}}=\left[\begin{array}{cc}
0 & |\mathbf{k}| \\
-|\mathbf{k}| & 0
\end{array}\right]\left[\begin{array}{c}
\hat{z} \\
\hat{p}
\end{array}\right], \hat{F}^{(3)}\left(\hat{\mathbf{U}}^{3}\right)=\frac{-\mathrm{i} \alpha k_{1} \widehat{|\mathbf{k}|} \widehat{\left(z^{3}\right)}\left[\begin{array}{l}
0 \\
1
\end{array}\right],}{\widehat{\left(z^{3}\right)}(\mathbf{k})=\frac{1}{(2 \pi)^{2 d}} \int_{\mathbf{k}^{\prime}, \mathbf{k}^{\prime \prime} \in \mathbb{R}^{2 d} ; \mathbf{k}^{\prime}+\mathbf{k}^{\prime \prime}+\mathbf{k}^{\prime \prime \prime}=\mathbf{k}} \hat{z}\left(\mathbf{k}^{\prime}\right) \hat{z}\left(\mathbf{k}^{\prime \prime}\right) \hat{z}\left(\mathbf{k}^{\prime \prime \prime}\right) \mathrm{d} \mathbf{k}^{\prime} \mathrm{d} \mathbf{k}^{\prime \prime} .}
\end{gathered}
$$

Since the factor $\frac{k_{1}}{|\mathbf{k}|}$ is uniformly bounded and smooth for $|\mathbf{k}| \neq 0$ conditions (2.26) and (2.28) are satisfied. The eigenvalues and corresponding eigenvectors of $\mathbf{L}$ are given explicitly:

$$
\omega_{+}(\mathbf{k})=|\mathbf{k}|, \omega_{-}(\mathbf{k})=-|\mathbf{k}|, \mathbf{g}_{+}(\mathbf{k})=2^{-1 / 2}\left[\begin{array}{c}
-\mathrm{i} \\
1
\end{array}\right], \mathbf{g}_{-}(\mathbf{k})=2^{-1 / 2}\left[\begin{array}{c}
\mathrm{i} \\
1
\end{array}\right]
$$

Since the matrix $\mathbf{L}(\mathbf{k})$ is Hermitian, Condition 2.5] is satisfied. The singular set $\sigma$ consists of the single point $\mathbf{k}=\mathbf{0}$. Note that conclusions of Theorem 2.19 are applicable to equation 
(2.100) and consequently to (2.99). For instance, we take the initial data for (2.100) in the form (2.79)

$$
z(\mathbf{r}, 0)=h_{0}, p(\mathbf{r}, 0)=h_{1}, h_{j}=\sum_{l=1}^{n_{h}} \Psi_{j l}\left(\beta \mathbf{r}-\mathbf{r}_{l}\right) \mathrm{e}^{\mathrm{i} \mathbf{k}_{* l} \cdot \mathbf{r}}+c c, j=0,1,
$$

where $\Psi_{0 l}(\mathbf{r}), \Psi_{1 l}(\mathbf{r})$ are arbitrary Schwartz functions, cc means complex conjugate to the preceding terms. The points $\mathbf{r}_{l}$ are arbitrary. Note that terms corresponding to $\mathbf{k}_{* l}$ can be written using the basis (2.101) as

$$
\left[\begin{array}{c}
\Psi_{0 l} \\
\Psi_{1 l}
\end{array}\right] \mathrm{e}^{\mathrm{i} \mathbf{k}_{* l} \cdot \mathbf{r}}=\left[\Phi_{+, l} \mathbf{g}_{+}+\Phi_{-, l} \mathbf{g}_{-}\right] \mathrm{e}^{\mathrm{i} \mathbf{k}_{* l} \cdot \mathbf{r}} .
$$

In this case all requirements of Definition 2.9 are fulfilled. The number of initial wavepackets for the first-order system (2.100) corresponding to initial data (2.102) ) equals $N_{h}=2 n_{h}$ and there are $2 N_{h}$ wavepacket centers $\vartheta \mathbf{k}_{* l}, \vartheta= \pm$. To satisfy the requirements of Condition 2.14 we have to take the wave vectors $\mathbf{k}_{* l} \neq 0$ so that

$$
\frac{\vartheta \mathbf{k}_{* l}}{\left|\mathbf{k}_{* l}\right|} \neq \frac{\vartheta^{\prime} \mathbf{k}_{* l^{\prime}}}{\left|\mathbf{k}_{* l^{\prime}}\right|} \text { if } l \neq l^{\prime} \text { or } \vartheta \neq \vartheta^{\prime}
$$

which provides (2.41). Since

$$
\left|\mathbf{k}_{* l}\right| \lambda-\zeta\left|\lambda \mathbf{k}_{* l}\right|=\left|\mathbf{k}_{* l}\right|(\lambda-\zeta|\lambda|),
$$

equation (2.42) has solutions $\lambda \neq \zeta$ and every point $\mathbf{k}_{* l}$ does not satisfy Condition 2.13. This is the property of the very special, purely homogeneous $\omega(\mathbf{k})=|\mathbf{k}|$. Checking the second alternative, namely Condition 2.23 we observe that

$$
\nabla_{\mathbf{k}}\left|\nu \mathbf{k}_{* l}\right|=\frac{\nu \mathbf{k}_{* l}}{\left|\nu \mathbf{k}_{* l}\right|}=\frac{\nu}{|\nu|} \frac{\mathbf{k}_{* l}}{\left|\mathbf{k}_{* l}\right|} .
$$

Hence, if

$$
\frac{\vartheta \mathbf{k}_{* l}}{\left|\mathbf{k}_{* l}\right|} \neq \frac{\vartheta^{\prime} \mathbf{k}_{* l^{\prime}}}{\left|\mathbf{k}_{* l^{\prime}}\right|} \text { for } l \neq l^{\prime} \text { or } \vartheta \neq \vartheta^{\prime} \text { and if } \mathbf{k}_{* l} \neq 0
$$

then Condition 2.23 is satisfied and Superposition Theorem 2.19 is applicable. As a corollary of Theorem 2.19] applied to (2.99) we obtain that if the initial data for (2.99) equal the sum of wavepackets, then the solution equals the sum of separate solutions plus a small remainder, more precisely we have the following theorem.

Theorem 2.26 (superposition principle for wave equation) Assume that the initial data for (2.100) to be a multi-wavepacket of the form (2.102) and 2.46) holds. Then the solution $z(\mathbf{r}, \tau)$ to (2.100), (2.102) satisfy the superposition principle, namely

$$
z(\mathbf{r}, \tau)=\sum_{\vartheta= \pm} \sum_{l=1}^{n_{h}} z_{\vartheta, l}(\mathbf{r}, \tau)+\mathbf{D}_{1}(\mathbf{r}, \tau), p(\mathbf{r}, \tau)=\sum_{\vartheta= \pm} \sum_{l=1}^{n_{h}} p_{\vartheta, l}(\mathbf{r}, \tau)+\mathbf{D}_{2}(\mathbf{r}, \tau)
$$


where $z_{\vartheta, l}(\mathbf{r}, \tau), p_{\vartheta, l}(\mathbf{r}, \tau)$ is a solution of (2.100) with the initial condition

$$
\left[\begin{array}{c}
z_{\vartheta, l}(\mathbf{r}, 0) \\
p_{\vartheta, l}(\mathbf{r}, 0)
\end{array}\right]=\Phi_{\vartheta, l}\left(\beta \mathbf{r}-\mathbf{r}_{l}\right) \mathbf{g}_{\vartheta} \mathrm{e}^{\mathrm{i} \mathbf{k}_{* l} \cdot \mathbf{r}}+\mathrm{cc}
$$

with $\Phi_{\vartheta, l}(\mathbf{r})$ being arbitrary Schwartz functions. If (2.104) holds, the coupling terms $\mathbf{D}_{1}$ and $\mathbf{D}_{2}$ satisfy the bound

$$
\sup _{0 \leq \tau \leq \tau_{*}}\left[\left\|\mathbf{D}_{1}(\tau)\right\|_{L_{\infty}}+\left\|\mathbf{D}_{2}(\tau)\right\|_{L_{\infty}}\right] \leq C_{\delta}^{\prime} \frac{\varrho}{\beta^{1+\delta}}
$$

where $\tau_{*}$ and $C_{\delta}^{\prime}$ do not depend on $\beta, \varrho$ and $\delta$ can be taken arbitrary small..

In the following sections we introduce concepts and develop analytic tools allowing to prove the approximate linear superposition principle as stated in Theorems [2.15] 2.19] and 2.25 .

\section{Reduced evolution equation}

Since the properties of the evolution equations (2.3) and (2.61) are very similar, we consider here in detail the lattice evolution equation (2.3) with understanding that all the statements apply to the PDE (2.61) if we replace $\tilde{\mathbf{U}}$ with $\hat{\mathbf{U}},[-\pi, \pi]^{d}$ with $\mathbb{R}^{d}$, the function space $L_{1}=L_{1}\left([-\pi, \pi]^{d}\right)$ with $L_{1}=L_{1}\left(\mathbb{R}^{d}\right)$ and so on.

First, using the variation of constants formula we recast the modal evolution equation (2.3) into the following equivalent integral form

$$
\tilde{\mathbf{U}}(\mathbf{k}, \tau)=\int_{0}^{\tau} \mathrm{e}^{\frac{-\mathrm{i}\left(\tau-\tau^{\prime}\right)}{\varrho} \mathbf{L}(\mathbf{k})} \tilde{F}(\tilde{\mathbf{U}})(\mathbf{k}, \tau) d \tau^{\prime}+\mathrm{e}^{\frac{-\mathrm{i} \zeta \tau}{\varrho} \mathbf{L}(\mathbf{k})} \tilde{\mathbf{h}}(\mathbf{k}), \tau \geq 0 .
$$

Then we introduce for $\tilde{\mathbf{U}}(\mathbf{k}, \tau)$ its two-time-scale representation (with respectively slow and fast times $\tau$ and $t=\frac{\tau}{\varrho}$ )

$$
\tilde{\mathbf{U}}(\mathbf{k}, \tau)=\mathrm{e}^{-\frac{\mathrm{i} \tau}{\varrho} \mathbf{L}(\mathbf{k})} \tilde{\mathbf{u}}(\mathbf{k}, \tau), \tilde{\mathbf{U}}_{n, \zeta}(\mathbf{k}, \tau)=\tilde{\mathbf{u}}_{n, \zeta}(\mathbf{k}, \tau) \mathrm{e}^{-\frac{\mathrm{i} \tau}{\varrho} \zeta \omega_{n}(\mathbf{k})},
$$

where $\tilde{\mathbf{u}}_{n, \zeta}(\mathbf{k}, \tau)$ are the modal coefficients of $\tilde{\mathbf{u}}(\mathbf{k}, \tau)$ (see (2.18) $)$; note that $\tilde{\mathbf{u}}_{n, \zeta}(\mathbf{k}, \tau)$ may depend on $\varrho$, therefore (3.2) is just a change of variables. Consequently we obtain the following reduced evolution equation for $\tilde{\mathbf{u}}=\tilde{\mathbf{u}}(\mathbf{k}, \tau), \tau \geq 0$,

$$
\begin{aligned}
& \tilde{\mathbf{u}}(\mathbf{k}, \tau)=\mathcal{F}(\tilde{\mathbf{u}})(\mathbf{k}, \tau)+\tilde{\mathbf{h}}(\mathbf{k}), \mathcal{F}(\tilde{\mathbf{u}})=\sum_{m=2}^{m_{F}} \mathcal{F}^{(m)}\left(\tilde{\mathbf{u}}^{m}(\mathbf{k}, \tau)\right), \\
& \mathcal{F}^{(m)}\left(\tilde{\mathbf{u}}^{m}\right)(\mathbf{k}, \tau)=\int_{0}^{\tau} \mathrm{e}^{\frac{\mathrm{i} \tau^{\prime}}{\varrho} \mathbf{L}(\mathbf{k})} \tilde{F}^{(m)}\left(\left(\mathrm{e}^{\frac{-\mathrm{i} \tau^{\prime}}{\varrho} \mathbf{L}(\cdot)} \tilde{\mathbf{u}}\right)^{m}\right)\left(\mathbf{k}, \tau^{\prime}\right) \mathrm{d} \tau^{\prime},
\end{aligned}
$$


where the quantities $\tilde{F}^{(m)}$ are defined by (2.21) and (2.22) in terms of the susceptibilities $\chi^{(m)}$.

The norm of the oscillatory integral $\mathcal{F}^{(m)}$ in (3.4) is estimated in terms of the norm of the tensor $\chi^{(m)}(\mathbf{k}, \vec{k})$ defined in (2.26),$(2.27)$. The operator $\mathcal{F}^{(m)}$ is shown to be a bounded one from $(E)^{m}$ into $E$, see Lemma 5.1 for details The proof of this property is based on the following Young inequality for the convolution

$$
\|\tilde{\mathbf{u}} * \tilde{\mathbf{v}}\|_{L_{1}} \leq\|\tilde{\mathbf{u}}\|_{L_{1}}\|\tilde{\mathbf{v}}\|_{L_{1}}
$$

For a detailed analysis of solutions of (3.3) we recast the equation (3.3) for $\tilde{\mathbf{u}}(\mathbf{k}, \tau)$ using projections (2.19) as the following expanded reduced evolution equation

$$
\tilde{\mathbf{u}}_{n, \zeta}(\mathbf{k}, \tau)=\sum_{m=2}^{\infty} \sum_{\vec{n}, \vec{\zeta}} \mathcal{F}_{n, \zeta, \vec{n}, \vec{\zeta}}^{(m)}\left(\tilde{\mathbf{u}}^{m}\right)(\mathbf{k}, \tau)+\mathbf{h}_{n, \zeta}(\mathbf{k}), \tau \geq 0
$$

for the modal coefficient $\tilde{\mathbf{u}}_{n, \zeta}(\mathbf{k}, \tau)$. In the above formula and elsewhere we use notations

$$
\vec{n}=\left(n^{\prime}, \ldots, n^{(m)}\right), \vec{\zeta}=\left(\zeta^{\prime}, \ldots, \zeta^{(m)}\right), \vec{k}=\left(\mathbf{k}^{\prime}, \ldots, \mathbf{k}^{(m)}\right) .
$$

The operators $\mathcal{F}_{n, \zeta, \vec{n}, \vec{\zeta}}^{(m)}$ are $m$-linear oscillatory integral operators defined by the formulas

$$
\begin{gathered}
\mathcal{F}_{n, \zeta, \vec{n}, \vec{\zeta}}^{(m)}\left(\tilde{\mathbf{u}}_{1} \ldots \tilde{\mathbf{u}}_{m}\right)(\mathbf{k}, \tau)=\int_{0}^{\tau} \int_{\mathbb{D}_{m}} \exp \left\{\mathrm{i} \phi_{n, \zeta, \vec{n}, \vec{\zeta}}(\mathbf{k}, \vec{k}) \frac{\tau_{1}}{\varrho}\right\} \\
\chi_{n, \zeta, \vec{n}, \vec{\zeta}}^{(m)}(\mathbf{k}, \vec{k})\left[\tilde{\mathbf{u}}_{1}\left(\mathbf{k}^{\prime}, \tau_{1}\right), \ldots, \tilde{\mathbf{u}}_{m}\left(\mathbf{k}^{(m)}(\mathbf{k}, \vec{k}), \tau_{1}\right)\right] \tilde{\mathrm{d}}^{(m-1) d} \vec{k} \mathrm{~d} \tau_{1},
\end{gathered}
$$

where we use notations (2.23), (2.24), (2.25). In (3.8) the interaction phase function $\phi$ is defined by

$$
\phi_{n, \zeta, \vec{n}, \vec{\zeta}}(\mathbf{k}, \vec{k})=\zeta \omega_{n}(\mathbf{k})-\zeta^{\prime} \omega_{n^{\prime}}\left(\mathbf{k}^{\prime}\right)-\ldots-\zeta^{(m)} \omega_{n^{(m)}}\left(\mathbf{k}^{(m)}\right), \mathbf{k}^{(m)}=\mathbf{k}^{(m)}(\mathbf{k}, \vec{k})
$$

and the susceptibilities $\chi_{n, \zeta, \vec{n}, \vec{\zeta}}^{(m)}(\mathbf{k}, \vec{k})$ are $m$-linear symmetric tensors (i.e. mappings from $\left(\mathbb{C}^{2 J}\right)^{m}$ into $\mathbb{C}^{2 J}$ ) defined for almost all $\mathbf{k}, \vec{k}$ by the following formula

$$
\begin{gathered}
\chi_{n, \zeta, \vec{n}, \vec{\zeta}}^{(m)}(\mathbf{k}, \vec{k})\left[\tilde{\mathbf{u}}_{1}\left(\mathbf{k}^{\prime}\right), \ldots, \tilde{\mathbf{u}}_{m}\left(\mathbf{k}^{(m)}\right)\right]= \\
\Pi_{n, \zeta}(\mathbf{k}) \chi^{(m)}(\mathbf{k}, \vec{k})\left[\Pi_{n^{\prime}, \zeta^{\prime}}\left(\mathbf{k}^{\prime}\right) \tilde{\mathbf{u}}_{1}\left(\mathbf{k}^{\prime}\right), \ldots, \Pi_{n^{(m)}, \zeta^{(m)}}\left(\mathbf{k}^{(m)}(\mathbf{k}, \vec{k})\right) \tilde{\mathbf{u}}_{m}\left(\mathbf{k}^{(m)}(\mathbf{k}, \vec{k})\right)\right] .
\end{gathered}
$$

For the lattice equation $\chi_{n, \zeta, \vec{n}, \vec{\zeta}}^{(m)}(\mathbf{k}, \vec{k})$ is $2 \pi$-periodic with respect to every variable $\mathbf{k}, \mathbf{k}^{\prime}, \ldots, \mathbf{k}^{(m)}$. Note that operators $\mathcal{F}^{(m)}\left(\mathbf{u}^{m}\right)$ in (3.3) can be rewritten using (3.8) as

$$
\mathcal{F}^{(m)}\left(\mathbf{u}^{m}\right)=\sum_{\vec{n}, \vec{\zeta}} \mathcal{F}_{n, \zeta, \vec{n}, \vec{\zeta}}^{(m)}\left(\tilde{\mathbf{u}}^{m}\right) .
$$

We also call operators $\mathcal{F}_{n, \zeta, \vec{n}, \vec{\zeta}}^{(m)}$ decorated operators. 
Remark 3.1 The expanded reduced evolution equation (3.6) is instrumental to the nonlinear analysis. Its very form, a convergent series of multilinear forms which are oscillatory integrals (3.8), is already a significant step in the analysis of the solution accomplishing several tasks: (i) it suggests a constructive representation for the solution; (ii) every term $\mathcal{F}_{n, \zeta, \vec{n}, \vec{\zeta}}^{(m)}$ can be naturally interpreted as nonlinear interaction of the underlying linear modes; (iii) the representation of $\mathcal{F}_{n, \zeta, \vec{n}, \vec{\zeta}}^{(m)}$ as the oscillatory integral (3.8) involving the interaction phase $\phi_{n, \zeta, \vec{n}, \vec{\zeta}}$ and the susceptibilities $\chi_{n, \zeta, \vec{n}, \vec{\zeta}}^{(m)}(\mathbf{k}, \vec{k})$ directly relates $\mathcal{F}_{n, \zeta, \vec{n}, \vec{\zeta}}^{(m)}$ to the terms of the original evolution equation as well as to physically significant quantities. We can also add that since we consider $\varrho \rightarrow 0$ the interaction phase function $\phi_{n, \zeta, \vec{n}, \vec{\zeta}}(\mathbf{k}, \vec{k})$ plays the decisive role in the analysis of nonlinear interactions of different modes.

The analysis of fundamental properties of the reduced evolution equation (3.6), including, in particular, the linear modal superposition principle, involves and combines the following three components: (i) the linear spectral theory component in the form of the modal decomposition of the solution and introduction of wavepackets as elementary waves; (ii) functionanalytic component which deals with the structure of series similar to the one in (3.6) and its dependence on the nonlinearity of the original evolution equation; (iii) asymptotic analysis of oscillatory integrals (3.8) which allows to estimate the magnitude of nonlinear interactions between different modes and, in particular, to show that generically different modes almost do not interact leading to the superposition principle.

Sometimes it is convenient to rewrite (3.8) in a slightly different form. The convolution integral (3.8) according to (2.25) involves the following phase matching condition

$$
\mathbf{k}^{\prime}+\ldots+\mathbf{k}^{(m)}=\mathbf{k} .
$$

Using the following notation for the integral over the plane (3.12)

$$
\begin{array}{r}
\int_{\mathbf{k}^{\prime}, \ldots, \mathbf{k}^{(m-1)} \in[-\pi, \pi]^{(m-1) d} ; \mathbf{k}^{\prime}+\ldots+\mathbf{k}^{(m)}=\mathbf{k}} f(\mathbf{k}, \vec{k}) \mathrm{d} \mathbf{k}^{\prime} \ldots \mathrm{d} \mathbf{k}^{(m-1)}= \\
\int_{[-\pi, \pi]^{m d}} f(\mathbf{k}, \vec{k}) \delta\left(\mathbf{k}-\mathbf{k}^{\prime}-\ldots-\mathbf{k}^{(m)}\right) \mathrm{d} \mathbf{k}^{\prime} \ldots \mathrm{d} \mathbf{k}^{(m)}
\end{array}
$$

in terms of a delta-function we can rewrite (3.8) in the form

$$
\begin{aligned}
& \mathcal{F}_{n, \zeta, \vec{n}, \vec{\zeta}}^{(m)}\left(\tilde{\mathbf{u}}_{1} \ldots \tilde{\mathbf{u}}_{m}\right)(\mathbf{k}, \tau)=\frac{1}{(2 \pi)^{m(d-1)}} \int_{0}^{\tau} \int_{[-\pi, \pi]^{m d}} \exp \left\{\mathrm{i} \phi_{n, \zeta, \vec{n}, \vec{\zeta}}(\mathbf{k}, \vec{k}) \frac{\tau_{1}}{\varrho}\right\} \\
& \delta\left(\mathbf{k}-\mathbf{k}^{\prime}-\ldots-\mathbf{k}^{(m)}\right) \chi_{n, \zeta, \vec{n}, \vec{\zeta}}^{(m)}(\mathbf{k}, \vec{k}) \tilde{\mathbf{u}}_{1, \zeta^{\prime}}\left(\mathbf{k}^{\prime}\right) \ldots \tilde{\mathbf{u}}_{m, \zeta^{(m)}}\left(\mathbf{k}^{(m)}\right) \mathrm{d} \mathbf{k}^{\prime} \ldots \mathrm{d} \mathbf{k}^{(m)} d \tau_{1} .
\end{aligned}
$$

\section{Function-analytic operator series}

In this section necessary algebraic concepts required for the analysis are introduced. We study the reduced evolution equation (3.3) as a particular case of the following abstract nonlinear 
equation in a Banach space

$$
\mathbf{u}=\mathcal{F}(\mathbf{u})+\mathbf{x}, \mathcal{F}(\mathbf{u})=\sum_{s=2}^{\infty} \mathcal{F}^{(s)}\left(\mathbf{x}^{s}\right)
$$

where the nonlinearity $\mathcal{F}(\mathbf{u})$ is an analytic operator represented by a convergent operator series. It is well known (see [25]) that the solution $\mathbf{u}=\mathcal{G}(\mathbf{x})$ of such equation can be represented as a convergent series in terms of $m$-linear operators $\mathcal{G}_{m}$ which are constructed based on $\mathcal{F}$ :

$$
\begin{gathered}
\mathcal{G}(\mathbf{x})=\mathcal{G}(\mathcal{F}, \mathbf{x})=\sum_{m=1}^{\infty} \mathcal{G}^{(m)}\left(\mathbf{x}^{m}\right), \mathcal{G}^{(m)}\left(\mathbf{x}^{m}\right)=\mathcal{G}^{(m)}\left(\mathcal{F}, \mathbf{x}^{m}\right), \text { where } \\
\mathbf{x}^{m}=\underbrace{\mathbf{x} \ldots \mathbf{x}}_{m \text { times }}=\underbrace{\mathbf{x} \ldots \mathbf{x}}_{m \text { times }} .
\end{gathered}
$$

Using the multilinearity of $\mathcal{G}^{(m)}$ we readily obtain the formula

$$
\begin{gathered}
\mathcal{G}\left(\mathbf{x}_{1}+\ldots+\mathbf{x}_{N}\right)=\sum_{m=1}^{\infty} \mathcal{G}^{(m)}\left(\left(\mathbf{x}_{1}+\ldots+\mathbf{x}_{N}\right)^{m}\right) \\
=\sum_{m=1}^{\infty} \mathcal{G}\left(\left(\mathbf{x}_{1}\right)^{m}\right)+\ldots+\sum_{m=1}^{\infty} \mathcal{G}\left(\left(\mathbf{x}_{N}\right)^{m}\right)+\mathcal{G}_{\mathrm{CI}}\left(\mathbf{x}_{1}, \ldots, \mathbf{x}_{N}\right),
\end{gathered}
$$

where $\mathbf{x}=\mathbf{x}_{1}+\ldots+\mathbf{x}_{N}$ represents a multi-wavepacket and $\mathcal{G}_{\mathrm{CI}}\left(\mathbf{x}_{1}, \ldots, \mathbf{x}_{N}\right)$ collects all "cross terms" and describes the "cross interaction" (CI) of involved wavepackets $\mathbf{x}_{1}, \ldots, \mathbf{x}_{N}$. We will find in sufficient detail the dependence of the solution operators $\mathcal{G}_{m}$ on the nonlinearity $\mathcal{F}$ and prepare a basis for the consequent estimation of nonlinear interactions between different modes and wavepackets. Then combining the facts about the structure of the solution operators $\mathcal{G}^{(m)}$ with asymptotic estimates of relevant oscillatory integrals we show that for a multi-wavepacket $\mathbf{x}=\mathbf{x}_{1}+\ldots+\mathbf{x}_{N}$ the cross-interaction term satisfies the following estimate

$$
\left\|\mathcal{G}_{\mathrm{CI}}\left(\mathbf{x}_{1}, \ldots, \mathbf{x}_{N}\right)\right\|=O(\beta)+O\left(\varrho|\ln \beta| / \beta^{1+\epsilon}\right), \beta, \varrho \rightarrow 0
$$

implying the modal superposition principle.

\subsection{Multilinear forms and polynomial operators}

The analysis of nonlinear equations of the form (3.3) requires the use of appropriate Banach spaces of time dependent fields, as well as multilinear and analytic functions in those spaces. It also uses an appropriate version of the implicit function theorem. For the reader's convenience we collect in this section the known concepts and statements on the above-mentioned subjects needed for our analysis. We in this section consider functional-analytic operators which are defined in a ball in a Banach space $X$ with the norm $\|\mathbf{x}\|_{X}$. In our treatment of the analytic functions in infinitely-dimensional Banach spaces we follow to [25, Section 3], 21. 
Definition 4.1 (polylinear operator) Suppose that $\mathbf{x}_{1}, \mathbf{x}_{2}, \ldots, \mathbf{x}_{n}$ are vectors in a Banach space $X$. Let a function $\mathcal{F}^{(n)}(\vec{x}), \vec{x}=\left(\mathbf{x}_{1}, \ldots, \mathbf{x}_{n}\right)$, take values in $X$ and be defined for all $\vec{x} \in X^{n}$. Such a function $\mathcal{F}^{(n)}$ is called a $n$-linear operator if it is linear in each variable, and it is said to be bounded if its following norm is finite

$$
\left\|\mathcal{F}^{(n)}\right\|=\sup _{\left\|\mathbf{x}_{1}\right\|_{X}=\ldots=\left\|\mathbf{x}_{n}\right\|_{X}=1}\left\|\mathcal{F}^{(n)}\left(\mathbf{x}_{1} \mathbf{x}_{2} \ldots \mathbf{x}_{n}\right)\right\|_{X}<\infty .
$$

Definition 4.2 (polynomial) A function $P(x)$ from $X$ to $X$ defined for all $x \in X$ is called a polynomial in $\mathbf{x}$ of degree $n$ if for all $\mathbf{a}, \mathbf{h} \in X$ and all complex $\alpha$

$$
P(\mathbf{a}+\alpha \mathbf{h})=\sum_{\nu=0}^{n} P_{\nu}(\mathbf{a}, \mathbf{h}) \alpha^{\nu},
$$

where $P_{\nu}(a, h) \in X$ are independent of $\alpha$. The degree of $P_{n}$ is exactly $n$ if $P_{n}(a, h)$ is not identically zero. A polynomial $\mathcal{F}(\mathbf{x})$ is a homogeneous polynomial of a degree $n$ if for all $c \in \mathbb{C}$

$$
\mathcal{F}(c \mathbf{x})=c^{n} \mathcal{F}(\mathbf{x})
$$

Then $n$ is called also the homogeneity index of $\mathcal{F}(\mathbf{x})$. A homogeneous polynomial $\mathcal{F}$ is called bounded if its norm

$$
\|\mathcal{F}\|_{*}=\sup _{\|x\|_{X}=1}\left\{\|\mathcal{F}(\mathbf{x})\|_{X}\right\}
$$

is finite. For a given n-linear operator $\mathcal{F}^{(n)}(\vec{x})=\mathcal{F}^{(n)}\left(\mathbf{x}_{1} \mathbf{x}_{2} \ldots \mathbf{x}_{n}\right)$ we denote by $\mathcal{F}^{(n)}\left(\mathbf{x}^{n}\right)$ a homogeneous of degree $n$ polynomial from $X$ to $X$ :

$$
\mathcal{F}^{(n)}\left(\mathbf{x}^{n}\right)=\mathcal{F}^{(n)}(\mathbf{x} \ldots \mathbf{x}) .
$$

Note the norm definitions (4.3), (4.4) and (4.5) readily imply

$$
\left\|\mathcal{F}^{(n)}\right\|_{*} \leq\left\|\mathcal{F}^{(n)}\right\| \text {. }
$$

Definition 4.3 (analyticity class 1 ) Let a function $\mathcal{F}$ be defined by the following convergent series

$$
\mathcal{F}(x)=\sum_{m=2}^{\infty} \mathcal{F}^{(m)}\left(\mathbf{x}^{m}\right) \text { for }\|\mathbf{x}\|_{X}<R_{* \mathcal{F}},
$$

where $\mathcal{F}^{(m)}\left(\mathbf{x}^{m}\right), m=2,3, \ldots$ is a sequence of bounded $m$-homogenious polynomials satisfying

$$
\left\|\mathcal{F}^{(m)}\right\|_{*} \leq C_{* \mathcal{F}} R_{* \mathcal{F}}^{-m}, m=2,3, \ldots,
$$

Then we say that $\mathcal{F}(x)$ belongs to the analyticity class $A_{*}\left(C_{* \mathcal{F}}, R_{* \mathcal{F}}\right)$ and write $\mathcal{F} \in A_{*}\left(C_{* \mathcal{F}}, R_{* \mathcal{F}}\right)$.

Notice that for $\|\mathbf{x}\|_{X}<R_{* \mathcal{F}}$ we have

$$
\|\mathcal{F}(\mathbf{x})\|_{X} \leq C_{* \mathcal{F}} \sum_{n=2}^{\infty}\|\mathbf{x}\|_{X}^{n} R_{* \mathcal{F}}^{-n} \leq C_{* \mathcal{F}} \frac{\|\mathbf{x}\|_{X}^{n_{0}} R_{* \mathcal{F}}^{-n_{0}}}{1-\|\mathbf{x}\|_{X} R_{* \mathcal{F}}^{-1}},
$$

implying, in particular, the convergence of the series (4.7). 
Definition 4.4 (analyticity class 2) If $\mathcal{F}^{(m)}(\vec{x}), m=2,3, \ldots$, is a sequence of bounded $m$-linear operators from $X^{m}$ to $X$ and

$$
\left\|\mathcal{F}^{(m)}\right\| \leq C_{\mathcal{F}} R_{\mathcal{F}}^{-m}, m=2,3 \ldots,
$$

we say that a function $\mathcal{F}$ defined by the series (4.7) for $\|x\|_{X}<R_{\mathcal{F}}$ belongs to the analyticity class $A\left(C_{\mathcal{F}}, R_{\mathcal{F}}\right)$ and write $\mathcal{F} \in A\left(C_{\mathcal{F}}, R_{\mathcal{F}}\right)$.

In this paper we will use operators from the classes $A\left(C_{\mathcal{F}}, R_{\mathcal{F}}\right)$ based on multilinear operators.

Note that evidently $A\left(C_{\mathcal{F}}, R_{\mathcal{F}}\right) \subset A_{*}\left(C_{\mathcal{F}}, R_{\mathcal{F}}\right)$. One can construct a polynomial based on a multilinear operator according to the formula (4.5). Conversely, the construction of a multilinear operator, called polar form, based on a given homogeneous polynomial is described by the following statement, [21, Section 1.1, 1.3], [25, Section 26.2].

Proposition 4.5 (polar form) For any homogeneous polynomial $P^{(n)}(x)$ of degree $n$ there is a unique symmetric $n$-linear operator $\tilde{P}^{(n)}\left(\mathbf{x}_{1} \mathbf{x}_{2} \ldots \mathbf{x}_{n}\right)$, called the polar form of $P_{n}(\mathbf{x})$, such that $P^{(n)}(\mathbf{x})=\tilde{P}^{(n)}(\mathbf{x} \ldots \mathbf{x})$. It is defined by the following polarization formula:

$$
\tilde{P}^{(n)}\left(\mathbf{x}_{1} \mathbf{x}_{2} \ldots \mathbf{x}_{n}\right)=\frac{1}{2^{n} n !} \sum_{\xi_{j}= \pm 1} P^{(n)}\left(\sum_{j=1}^{n} \xi_{j} \mathbf{x}_{j}\right)
$$

In addition to that, the following estimate holds:

$$
\left\|P_{n}\right\|_{*} \leq\left\|\tilde{P}_{n}\right\| \leq \frac{n^{n}}{n !}\left\|P_{n}\right\|_{*} \leq \mathrm{e}^{n}\left\|P^{(n)}\right\|_{*} .
$$

Since by Definition 4.4 functions from $A(C, R)$ have zero of the second order at zero, their Lipschitz constant is small in a vicinity of zero. More exactly, the following statement holds.

Lemma 4.6 (Lipschitz estimate) If $\mathcal{F} \in A\left(C_{\mathcal{F}}, R_{\mathcal{F}}\right)$ then

$$
\|\mathcal{F}(\mathbf{x})-\mathcal{F}(\mathbf{y})\| \leq C_{\mathcal{F}} C\|\mathbf{x}-\mathbf{y}\|(\|\mathbf{x}\|+\|\mathbf{y}\|) \text { for }\|\mathbf{x}\|,\|\mathbf{y}\| \leq R_{\mathcal{F}}^{\prime}<R_{\mathcal{F}}
$$

where $C>0$ depends on $R_{\mathcal{F}}^{\prime}$ and $R_{\mathcal{F}}$.

\subsection{Implicit Function Theorem and expansion of operators into composition monomials}

Here we provide a version of the Implicit Function Theorem, first we formulate classical implicit function theorem for equations $\mathbf{u}=\mathcal{F}(\mathbf{u})+\mathbf{x}$ with analytic function $\mathcal{F}$ and then we present a refined implicit function theorem. The refined implicit function theorem we prove here produces expansion of the solution $\mathbf{u}$ into a sum of terms which are multi-linear not only 
with respect to $\mathbf{x}$ but also with respect to $\mathcal{F}$. The formulation of the theorem and the proof involve convenient labeling of the terms of the expansion (called composition monomials), and we use properly introduced trees to this end. The explicit expansion produced by the refined implicit function theorem is required to be able to take into account rather subtle mechanisms which lead to the superposition principle.

Let us consider the abstract nonlinear equation (4.1) and its solution $\mathbf{u}=\mathbf{u}(\mathbf{x})$ for small $\|\mathbf{x}\|$ when the nonlinear operator $\mathcal{F}$ belongs to the class $A\left(C_{\mathcal{F}}, R_{\mathcal{F}}\right)$. We seek the solution $\mathbf{u}$ in the following form

$$
\mathbf{u}=\mathcal{G}(\mathcal{F}, \mathbf{x})=\sum_{m=1}^{\infty} \mathcal{G}^{(m)}\left(\mathbf{x}^{m}\right) \text { for sufficiently small }\|\mathbf{x}\|
$$

and we call $\mathcal{G}$ the solution operator for (4.1). It readily follows from (4.1) that

$$
\mathcal{G}(\mathcal{F}, \mathbf{x})=\mathbf{x}+\mathcal{F}(\mathcal{G}(\mathcal{F}, \mathbf{x}))
$$

and

$$
\sum_{m=1}^{\infty} \mathcal{G}^{(m)}\left(\mathbf{x}^{m}\right)=\mathbf{x}+\sum_{s=2}^{\infty} \mathcal{F}^{(s)}\left(\left(\sum_{m=1}^{\infty} \mathcal{G}^{(m)}\left(\mathbf{x}^{m}\right)\right)^{s}\right)
$$

¿From the above equation we can deduce recurrent formulas for multilinear operators $\mathcal{G}^{(m)}$. Indeed for $m=1$ the linear term is the identity operator

$$
\mathcal{G}^{(1)}(\mathbf{x})=\mathcal{F}^{(1)}(\mathbf{x}) \equiv \mathbf{x} .
$$

For $m \geq 2$ we write the following recurrent formula

$$
\mathcal{G}^{(m)}\left(\mathbf{x}_{1} \ldots \mathbf{x}_{m}\right)=\sum_{s=2}^{m} \sum_{i_{1}+\ldots+i_{s}=m} \mathcal{F}^{(s)}\left(\mathcal{G}^{\left(i_{1}\right)}\left(\mathbf{x}_{1} \ldots \mathbf{x}_{i_{1}}\right) \ldots \mathcal{G}^{\left(i_{s}\right)}\left(\mathbf{x}_{m-i_{s}+1} \ldots \mathbf{x}_{m}\right)\right) .
$$

By the construction, if multilinear operators $\mathcal{G}^{(i)}$ are defined by (4.18), then (4.16) is satisfied. Namely, expanding right-hand side of (4.16) using multi-linearity of $\mathcal{F}^{(s)}$ we obtain a sum of expressions as in right-hand side of (4.18), and since (4.18) holds, terms in the left-hand side of (4.16) with given homogeneity index $p$ cancel with the terms in the right-hand side with the same homogeneity. Note that in (4.18) we do not assume that the operators $\mathcal{F}^{(s)}$ and $\mathcal{G}^{(i)}$ are symmetrized and the order of variables is important; we prefer to treat $\mathcal{F}^{(s)}$ and $\mathcal{G}^{(m)}$ as multilinear operators of $s$ and $m$ variables respectively. Though, when we apply constructed $\mathcal{G}^{(i)}$ to solve (4.1), we set $\mathbf{x}_{1}=\ldots=\mathbf{x}_{m}$.

The following implicit function theorem holds (see [4] and Theorem 4.25 below with a similar proof).

Theorem 4.7 (Implicit Function Theorem) Let $\mathcal{F} \in A\left(C_{\mathcal{F}}, R_{\mathcal{F}}\right)$. Then there exists a solution $\mathbf{u}=\mathbf{x}+\mathcal{G}(\mathcal{F}, \mathbf{x})$ of the equation (4.1) $\mathbf{u}=\mathbf{x}+\mathcal{F}(\mathbf{u})$, given by the solution operator $\mathcal{G} \in A\left(C_{\mathcal{G}}, R_{\mathcal{G}}\right)$, where we can take

$$
C_{\mathcal{G}}=\frac{R_{\mathcal{F}}^{2}}{2\left(C_{\mathcal{F}}+R_{\mathcal{F}}\right)}, R_{\mathcal{G}}=\frac{R_{\mathcal{F}}^{2}}{4\left(C_{\mathcal{F}}+R_{\mathcal{F}}\right)}
$$


the series 4.14) converges for $\|\mathbf{x}\|_{X}<R_{\mathcal{G}}$. The multilinear operators $\mathcal{G}^{(m)}(\vec{x})$ satisfy the recursive relations (4.17), (4.18).

Note that uniqueness of the solution and continuous dependence on parameters follows from Lemma 4.6] and from a standard observation which we formulate in the following remark.

Remark 4.8 If $\mathbf{u}_{1}, \mathbf{u}_{2}$ are two solutions of the equation (4.1) with $\mathbf{x}=\mathbf{x}_{1}, \mathbf{x}_{2}$ respectively and $\left\|\mathbf{u}_{1}\right\|,\left\|\mathbf{u}_{2}\right\| \leq R$, and $\mathcal{F}(\mathbf{u})$ is Lipschitz continuous for $\|\mathbf{u}\| \leq R$ with a Lipschitz constant $q<1$ then $\left\|\mathbf{u}_{1}-\mathbf{u}_{2}\right\| \leq(1-q)^{-1}\left\|\mathbf{h}_{1}-\mathbf{h}_{2}\right\|$. If $\mathbf{u}_{1}, \mathbf{u}_{2}$ are two solutions of the equation (4.1) with $\mathcal{F}=\mathcal{F}_{0}$ and $\mathcal{F}=\mathcal{F}_{0}+\mathcal{F}_{1}$ respectively, $\left\|\mathbf{u}_{1}\right\|,\left\|\mathbf{u}_{2}\right\| \leq R$, and $\mathcal{F}(\mathbf{u})$ is Lipschitz continuous for $\|\mathbf{u}\| \leq R$ with a Lipschitz constant $q<1$ and $\mathcal{F}_{1}(\mathbf{u}) \leq \epsilon$ when $\|\mathbf{u}\| \leq R$ then $\left\|\mathbf{u}_{1}-\mathbf{u}_{2}\right\| \leq \epsilon(1-q)^{-1}$.

Observe that every term $\mathcal{G}^{\left(i_{l}\right)}$ in (4.18), in turn, can be recast as a sum (4.18) with $m$ replaced by $i_{l}<m$. Evidently applying the recurrent representation (4.18) and multilinearity of $\mathcal{F}^{(s)}$ we can get a formula for $\mathcal{G}^{(m)}$ as a sum of terms involving exclusively (i) the symbols $\mathcal{F}^{(m)}$, (ii) variables $\mathbf{x}_{j}$ and (iii) parentheses. We will refer to the terms of such a formula as composition monomials. To be precise we give below a formal recursive definition of composition monomials. The monomials are expressions which involve variables $\mathbf{u}_{j}, j=$ $1,2, \ldots$, and $m$-linear operators $\mathcal{F}^{(m)}, m=2,3 \ldots$, and are constructed by induction as follows.

Definition 4.9 (composition monomials) Let $\left\{\mathcal{F}^{(s)}\right\}_{s=2}^{\infty}$ be a sequence of s-linear operators which act on variables $\mathbf{u}_{j}, j=1,2, \ldots$ A composition monomial $M$ of rank 0 is the identity operator, namely $M\left(\mathbf{u}_{j}\right)=\mathbf{u}_{j}$, and its homogeneity index is 1 . A composition monomial $M$ of a non-zero rank $r \geq 1$ has the form

$$
M\left(\mathbf{u}_{i_{0}} \ldots \mathbf{u}_{i_{s}}\right)=\mathcal{F}^{(s)}\left(M_{1}\left(\mathbf{u}_{i_{0}} \ldots \mathbf{u}_{i_{1}}\right) \ldots M_{s}\left(\mathbf{u}_{i_{s-1}+1} \ldots \mathbf{u}_{i_{s}}\right)\right),
$$

where $M_{1}\left(\mathbf{u}_{i_{0}} \ldots \mathbf{u}_{i_{1}}\right), M_{2}\left(\mathbf{u}_{i_{1}+1} \ldots \mathbf{u}_{i_{2}}\right), \ldots, M_{s}\left(\mathbf{u}_{i_{s-1}+1} \ldots \mathbf{u}_{i_{s}}\right)$, with $1 \leq i_{0}<i_{1}<\ldots<i_{s}$, are composition monomials of ranks not exceeding $r-1$ (submonomials) and at least one of the rank $r-1$, the homogeneity index of $M_{j}$ equals $i_{j}-i_{j-1}$. For a composition monomial $M$ the operator $\mathcal{F}^{(s)}$ in its representation $(4.20)$ is called its root operator. The index of homogeneity of $M$ defined by (4.20) equals $i_{m}-i_{0}+1$. We call the labeling of the arguments of a composition monomial $M$ defined by (4.20) by consecutive integers standard labeling if $i_{0}=1$.

If the monomials $M_{1}, ., M_{s}$ have the respective homogeneity indexes $\nu\left(M_{i}\right)$ then we readily get that the homogeneity index of the monomial $M$ satisfies the identity

$$
\nu(M)=\nu\left(M_{1}\right)+\ldots+\nu\left(M_{s}\right) .
$$

Using the formula (4.20) inductively we find that any composition monomial $M$ is given by a formula which involves symbols from the set $\left\{\mathcal{F}^{(s)}\right\}_{s=2}^{\infty}$, arguments $\mathbf{u}_{i}$ and parentheses, and if $s$-linear operators are substituted as $\mathcal{F}^{(s)}$ we obtain the terms contained in the expansion of $\mathcal{G}^{(m)}$. 
Definition 4.10 (incidence number) The total number of symbols $\mathcal{F}^{(q)}$ involved in $M$ is called the incidence number for $M$.

For instance, the expression of the form

$$
M=\mathcal{F}^{(4)}\left(\mathbf{u}_{1} \mathbf{u}_{2} \mathbf{u}_{3} \mathcal{F}^{(3)}\left(\mathbf{u}_{4} \mathcal{F}^{(2)}\left(\mathbf{u}_{5} \mathbf{u}_{6}\right) \mathcal{F}^{(3)}\left(\mathbf{u}_{7} \mathbf{u}_{8} \mathbf{u}_{9}\right)\right)\right)
$$

is an example of a composition monomial $M$ of rank 3, incidence number 4 and homogeneity index 9. It has three submonomials. Namely, the first one is $\mathcal{F}^{(3)}\left(\mathbf{u}_{4} \mathcal{F}^{(2)}\left(\mathbf{u}_{5} \mathbf{u}_{6}\right) \mathcal{F}^{(3)}\left(\mathbf{u}_{7} \mathbf{u}_{8} \mathbf{u}_{9}\right)\right)$ of rank 2 and incidence number 3. The second submonomial $\mathcal{F}^{(2)}\left(\mathbf{u}_{5} \mathbf{u}_{6}\right)$ has rank 1 and incidence number 1 , and the third one is $\mathcal{F}^{(3)}\left(\mathbf{u}_{7} \mathbf{u}_{8} \mathbf{u}_{9}\right)$ of rank 1 and incidence number 1.

When analyzing the structure of composition monomials we use basic concepts and notation from the graph theory, namely, nodes, trees and subtrees.

Definition 4.11 (nodes, tree, subtree) $A$ (finite) directed graph $T$ consists of nodes $N_{i} \in N_{T}$ where $N_{T}$ is the set (finite) of nodes of $T$ and a set of edges $N_{i} N_{j} \in N_{T} \times N_{T}$. An edge $N_{i} N_{j}$ connects $N_{i}$ with $N_{j}$, it is an outcoming edge of $N_{i}$ and an incoming edge of $N_{j} . A$ tree (more precisely a rooted tree, we only consider rooted trees) is a directed connected graph which is cycle-free and has a selected root node, that is a node $N_{*}$ which has no incoming edges. If a node $N$ has an outcoming edge $N N_{j}$ the node $N_{j}$ is called a child node of $N$; if a node $N$ has an incoming edge $N_{j} N$ the node $N_{j}$ is called the parent node of $N$. We denote the parent node of $N$ by $p(N)$. If a node does not have children it is called an end node (or a leaf). For every node $N$ we denote by $\mu(N)$ the number of child nodes of the node $N$. If a path connects two nodes we call the number of edges in the path its length. We denote by $l(N)$ the length of a path which connects $N_{*}$ with $N$. Every node $N$ of the tree $T$ can be taken as a root node of a subtree which involves all descendent nodes of $N$ and connecting edges; we denote this maximal subtree $T^{\prime}(N)$. Since we consider only maximal subtrees we simply call them subtrees. We call by the rank of a tree the maximal length of a path from its root node to an end node and denote it by $r(T)$. We call by the rank of a node $N$ of the tree $T$ the rank of the subtree $T^{\prime}(N)$.

Definition 4.12 (tree incidence number and homogeneity index) For a tree $T$ we call the number of non-end nodes incidence number $i=i(T)$. We denote the number of end nodes of the tree by $\nu(T)$ and call it homogeneity index.

Elementary properties of trees. Since a tree does not have cycles, the path connecting two nodes on a tree is unique. The root node $N_{*}$ does not have a parent node, and since it is connected with every other node, every non-root node has a parent node. The end nodes have zero rank. The only node with $\operatorname{rank} r(T)$ is the root node. The total number of nodes of a tree $T$ equals $m(T)+i(T)$.

Definition 4.13 (ordered tree) $A$ tree is called an ordered tree if for every node $N$ all child nodes of $N$ are labeled by consecutive positive integers (which may start not from 1 ). 
Hence, for any node $N^{\prime} \neq N_{*}$ there is the parent node $N=p\left(N^{\prime}\right)$ and the order number (label) $o\left(N^{\prime}\right), i_{1} \leq o\left(N^{\prime}\right) \leq i_{1}+\mu(N)-1$. Two trees are equal if there is one-to-one mapping $\Theta$ between the nodes which preserves edges, maps the root node into the root node and preserves the order of children of every node up to a shift: if $\Theta(N)=\tilde{N}$ and $p\left(N_{1}\right)=p\left(N_{2}\right)=N$ then $o\left(N_{1}\right)-o\left(N_{2}\right)=o\left(\Theta\left(N_{1}\right)\right)-o\left(\Theta\left(N_{2}\right)\right)$.

Since we use in this paper only ordered trees we simply call them trees.

Standard node labeling and ordering. We use the following way of labeling and ordering of end nodes of a given ordered tree $T$. Let $\hat{r}$ be the rank of $T$. For any end node $N$ we take the unique path $N_{*} N_{1} \ldots N_{l(N)-1} N$ of length $l(N) \leq \hat{r}$ connecting it to the root. Since the tree is ordered, every node $N_{j}$ in the path has an order number $o\left(N_{j}\right)$. These order numbers form a word $w(N)$ of length $l(N)$. If $l(N)<\hat{r}$ we complete $w(N)$ to the length $\hat{r}$ adding several symbols $\infty$ and assuming that $\infty>n$ for $n=1,2, \ldots$ After that we order words $w(N)$ in the lexicographic order. We obtain the ordered list $w_{1}\left(N_{1}\right), \ldots, w_{\nu(T)}\left(N_{\nu(T)}\right)$. We take this ordering and labeling of the end nodes $N_{1}, \ldots, N_{\nu(T)}$ as a standard ordering and denote by $o_{0}(N)$ the consecutive number with respect to this labeling: $j=o_{0}\left(N_{j}\right)$. To label the nodes with rank $r$ we delete all the nodes of rank less than $r$ together with the incoming edges and nodes of rank $r$ become end nodes. We apply to them the described labeling and denote the indexes obtained by $o_{r}(N)$. Hence, every node $N$ of the tree $T$ has two integer numbers assigned: $r(N)$ and $o_{r(N)}(N)$. We introduce the standard labeling of all nodes of $T$ by applying the lexicographic ordering to pairs $\left(r(N), o_{r(N)}(N)\right)$, and denote the corresponding number $o(N), 1 \leq o(N) \leq m(T)+i(T)$.

The following statement follows straightforwardly from the definition of the standard ordering.

Proposition 4.14 If a tree $T$ has a subtree $T^{\prime}$ and the standard labeling of end nodes is used, then all the end nodes of the subtree $T^{\prime}$ fill an interval $j_{1} \leq o_{0}(N) \leq j_{2}$ for some $j_{1}$ and $j_{2}$.

Theorem 4.15 Let $\mathcal{T}_{2}$ be the set of ordered trees such that each node of a tree which is not an end node has at least two children nodes. The set of composition monomials based on $\left\{\mathcal{F}^{(s)}, s=2,3, \ldots\right\}$ is in one-to-one correspondence with the set $\mathcal{T}_{2}$. The correspondence has the following properties. The monomials of rank $r$ correspond to trees of rank $r$. The root node of the tree $T$ corresponds to the root operator of the composition monomial. The end nodes correspond to variables $\mathbf{u}_{j}, j=1, \ldots, \nu(T)$. The standard labeling of end nodes coincides with the consecutive labeling of the variables $\mathbf{u}_{j}$ of monomial from left to right. The homogeneity index of a monomial equals the homogeneity index of the corresponding tree. The incidence number of a monomial equals the incidence number of a tree, and the rank of a monomial equals the rank of a tree.

Proof. For a given $\left\{\mathcal{F}^{(s)}\right\}$ the set of monomials with rank $r$ is finite, the set of trees with rank $r$ is finite too. Therefore, to prove one-to-one correspondence of the two sets it is 
sufficient to construct two one-to-one mappings from the first set into the second and from the second into the first. First of all, using the induction with respect to $r$ we construct for every monomial the corresponding tree. Let $r=0$. A monomial of rank 0 has the form $\mathbf{u}_{1}$, and it corresponds to a tree involving one node. The tree has no edges and the node is the both the root and the end node; its incidence number is zero and homogeneity power is one. Assume now that we have defined a tree for any monomial of rank not greater than $r-1$. A monomial of rank $r$ has the form $\mathcal{F}^{(m)}\left(M_{1} \ldots M_{m}\right)$ where monomials $M_{1} \ldots M_{m}$ have rank not greater than $r-1$. Every monomial $M_{1} \ldots M_{m}$ corresponds to an ordered tree $T_{1}, \ldots, T_{m}$ with the root nodes $N_{* 1}, \ldots N_{* m}$. We form the tree $T$ as a union of the nodes of $T_{1}, \ldots, T_{m}$ and add one more node $N_{*}$ which corresponds to the root operator $\mathcal{F}^{(m)}$ and it becomes the root node of $T$. We take the union of edges from $T_{1}, \ldots, T_{m}$ and add $m$ more edges connecting $N_{*}$ with the nodes $N_{* 1}, \ldots N_{* m}$, the order of the nodes corresponds to ordering of $M_{1} \ldots M_{m}$ from left to right. The first mapping is constructed.

Now let us define for every ordered tree $T$ the corresponding monomial $M(\mathcal{F}, T)$. If we have a tree $T$ of rank zero we set $M(\mathcal{F}, T)=\mathbf{u}_{j}$ and $j=1$ if we use the standard labeling. Now we do induction step from $r-1$ to $r$. If we have a tree of rank $r$ we take the root node $N_{*}$ and its children $N_{* 1}, \ldots, N_{* s}, s=\mu\left(N_{*}\right)$. The subtrees $T^{\prime}\left(N_{* 1}\right), \ldots, T^{\prime}\left(N_{* s}\right)$ have rank not greater than $r-1$ and the monomials $M\left(\mathcal{F}, T^{\prime}\left(N_{* 1}\right)\right), \ldots, M\left(\mathcal{F}, T^{\prime}\left(N_{* s}\right)\right)$ are defined according to induction assumption, let $m\left(T^{\prime}\left(N_{* 1}\right)\right), \ldots, m\left(T^{\prime}\left(N_{* s}\right)\right)$ be their homogeneity indices. We set $m(T)=m\left(T^{\prime}\left(N_{* 1}\right)\right)+\ldots+m\left(T^{\prime}\left(N_{* s}\right)\right)$. We denote the variables of every monomial $M\left(\mathcal{F}, T^{\prime}\left(N_{* j}\right)\right)$ by $\mathbf{u}_{j, 1}, \ldots, \mathbf{u}_{j, m\left(T^{\prime}\left(N_{* j}\right)\right)}$ counting from left to right, and then labeling all the variables $\mathbf{u}_{j, l}$ using the lexicographic ordering of pairs $j, l$ we obtain variables $\mathbf{u}_{1}, \ldots, \mathbf{u}_{m(T)}$ and monomials

$$
M\left(\mathcal{F}, T^{\prime}\left(N_{* 1}\right)\right)\left(\mathbf{u}_{1}, \ldots, \mathbf{u}_{m\left(T^{\prime}\left(N_{* 1}\right)\right)}\right), M\left(\mathcal{F}, T^{\prime}\left(N_{* 2}\right)\right)\left(\mathbf{u}_{m_{1}+1}, \ldots, \mathbf{u}_{m_{1}+m_{2}}\right)
$$

etc., where $m_{j}=m\left(T^{\prime}\left(N_{* j}\right)\right)$. After that we set

$$
\begin{gathered}
M(\mathcal{F}, T)\left(\mathbf{u}_{1}, \ldots, \mathbf{u}_{m(T)}\right)= \\
\mathcal{F}^{(s)}\left(M\left(\mathcal{F}, T^{\prime}\left(N_{* 1}\right)\right)\left(\mathbf{u}_{1}, \ldots, \mathbf{u}_{m\left(T^{\prime}\left(N_{* 1}\right)\right)}\right), \ldots, M\left(\mathcal{F}, T^{\prime}\left(N_{* s}\right)\right)\left(\mathbf{u}_{m(T)-m_{s-1}+1}, \ldots, \mathbf{u}_{m(T)}\right)\right) .
\end{gathered}
$$

Note that the homogeneity index for the monomial $M$ equals the sum of the indices for submonomials $M_{1} \ldots M_{m}$, the homogeneity index for the tree $T$ equals the sum of the indices for subtrees $T_{1}, \ldots, T_{m}$, this implies their equality by induction. The incidence number for the monomial $M$ equals the sum of the numbers for submonomials $M_{1} \ldots M_{m}$ plus one; the incidence number for the tree $T$ equals the sum of the numbers for submonomials $T_{1}, \ldots, T_{m}$ plus one. Therefore, these quantities for monomials and trees are equal by induction. Induction is completed. Therefore we constructed the two mappings, one can easily check that they are one-to-one and have all required properties.

Definition 4.16 (monomial to a tree) For a tree $T \in \mathcal{T}_{2}$ we denote by $M(\mathcal{F}, T)$ the monomial which is constructed in Theorem 4.15. 
Conclusion 4.17 The above construction shows that the structure of every composition monomial is completely described by an (ordered) tree $T$ with nodes $N_{i}$ corresponding to the operators $\mathcal{F}^{\left(m_{i}\right)}$. At such a node $N_{i}$ (i) the number $m_{i}$ of outcoming edges equals the homogeneity index of $\mathcal{F}^{\left(m_{i}\right)}$; (ii) the outcoming edges are in one-to-one correspondence with the arguments of $\mathcal{F}^{\left(m_{i}\right)}$, and the ordering of the child nodes coincides with the ordering of arguments of $\mathcal{F}^{\left(m_{i}\right)}$ from left to right. The value of $m_{i}$ may be different for different nodes. A node corresponding to $\mathcal{F}^{(m)}$ is connected by edges with $m$ child nodes corresponding to the arguments of $\mathcal{F}^{(m)}$. Every node $N$ of the tree $T$ can be taken as a root node of a subtree $T^{\prime}(N)$ which correspond to a submonomial $M\left(\mathcal{F}, T^{\prime}(N)\right)$. Conversely, every submonomial of $M(\mathcal{F}, T)$ equals $M\left(\mathcal{F}, T^{\prime}(N)\right)$ for some mode $N$. If $m>1$ the submonomial has a nonzero rank. The number of non-end nodes equals to the number of symbols $\mathcal{F}^{(m)}$ used in $\mathcal{F}$-represenation of the monomial which is the incidence number of the monomial. The total number of end nodes of an $m$-homogenious operator equals to $m=\nu(T)$. The rank of a node $N$ equals the rank of the corresponding submonomial $M\left(\mathcal{F}, T^{\prime}(N)\right)$. The arguments $\mathbf{u}_{1}, \ldots \mathbf{u}_{s}$ of a monomial correspond to the end nodes of the tree. The standard labeling of nodes of $T$ agrees with the standard labeling (from left to right) of the arguments of the composition monomial $M(\mathcal{F}, T)$. The number of end nodes of the tree $T$ equals the homogeneity index of corresponding monomial. If the root mode of the tree $T$ of a monomial $M$ has $\mu\left(N_{*}\right)=m$ edges which are connected to child nodes $N_{1}, \ldots N_{m}$ then there is a node $\mathcal{F}^{\left(m_{j}\right)}, j=1, \ldots, n$ at the end of every edge such that $M$ has the form

$$
\mathcal{F}^{(m)}\left(\mathcal{F}^{\left(\mu\left(N_{1}\right)\right)}(\ldots), \ldots, \mathcal{F}^{\left(\mu\left(N_{m}\right)\right)}(\ldots)\right)
$$

Example 4.18 The tree corresponding to $\mathcal{F}^{(3)}\left(\mathbf{u}_{1} \mathbf{u}_{2} \mathcal{F}\left(\mathbf{u}_{1} \mathbf{u}_{2} \mathbf{u}_{3}\right)\right)$ has two nodes of non-zero rank, the root node of rank 2, one non-end node of rank 1 and five end nodes of rank 0 . Another example, the monomial (4.22) has the root node corresponding to $\mathcal{F}^{(4)}$, four edges lead respectively to nodes corresponding to the end nodes with $\mathbf{u}_{1}, \mathbf{u}_{2}, \mathbf{u}_{3}$ and to the non-end node with $\mathcal{F}^{(3)}$, see Fig. 2 .

Remark 4.19 Since all operators in the set $\left\{\mathcal{F}^{(s)}\right\}_{s=2}^{\infty}$ in 4.18) have the homogeneity index at least two, the trees of monomials generated by recurrent relations (4.18) have a special property: every non-end mode has at least two children.

Sometimes it is convenient to use monomials involving several types of operators. To describe such a situation we introduce for a given tree a decorated monomial.

Definition 4.20 (decorated monomial of a tree) Assume that we have several formal series $\left\{\mathcal{F}_{1}, \ldots, \mathcal{F}_{l}\right\}$ where $\mathcal{F}_{i}$ is represented by a formal series $\mathcal{F}_{l}=\sum_{m} \mathcal{F}_{i}^{(m)}, i=1, \ldots, l$. We call the set $\{\mathcal{F}\}=\left\{\mathcal{F}_{j}, j=1, \ldots, S\right\}$ the operator alphabet, and $j$ is called the decoration index. We consider a function $\Gamma(N), N \in T$, defined on the nodes of the tree $T$ and taking values in the set $\{1, \ldots, l\}$ of the decoration indices, and call such a function a decoration function on the tree $T$. Then for a decoration function $\Gamma(N)$ we define the decorated 


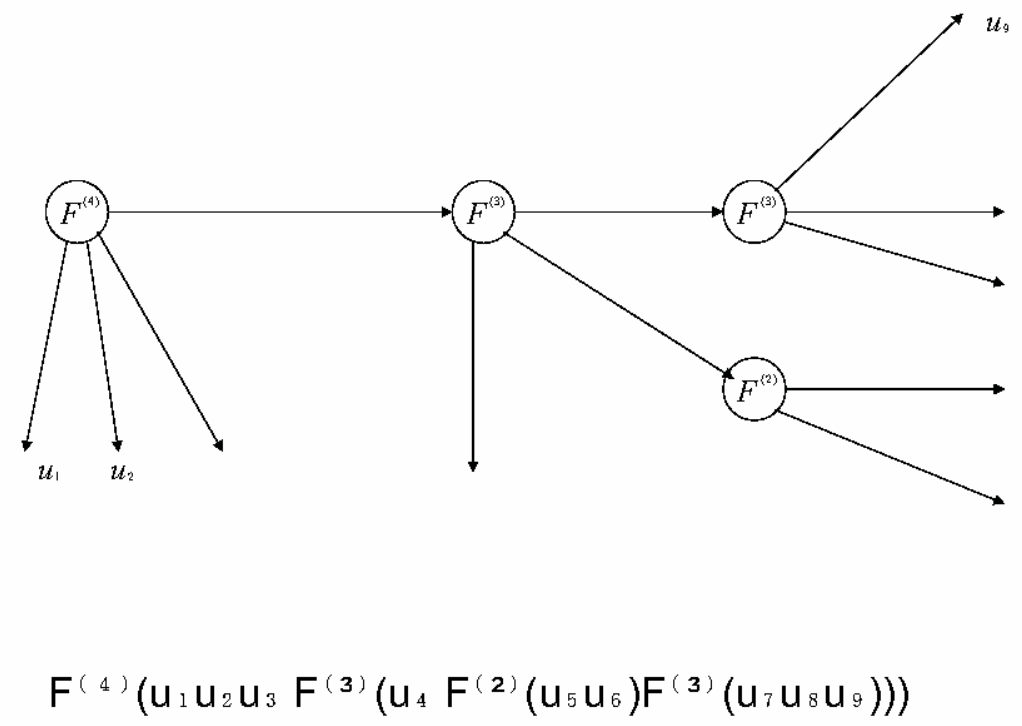

Figure 2: In this picture a tree corresponding to a monomial is drawn.

monomial $M(\{\mathcal{F}\}, \Gamma, T)$ of the tree $T$ by picking operators $\mathcal{F}_{j}^{(m)}$ with $j$ defined by $\Gamma$. For every node $N$ the homogeneity index $m=\mu(N)$ of the operator $\mathcal{F}_{j}^{(m)}$ equals to the number of children of $N$ and $j$ is defined by $\Gamma$, namely $\mathcal{F}_{j}, j=\Gamma(N)$.

Hence, a decorated monomial $M(\{\mathcal{F}\}, \Gamma, T)$ has instead of (4.23) the following form

$$
\mathcal{F}_{\Gamma(N)}^{(m)}\left(\mathcal{F}_{\Gamma\left(N_{1}\right)}^{\left(\mu\left(N_{1}\right)\right)}(\ldots), \ldots, \mathcal{F}_{\Gamma\left(N_{m}\right)}^{\left(\mu\left(N_{m}\right)\right)}(\ldots)\right) .
$$

When $\mathcal{F}_{i}^{(m)}$ are multilinear operators, a monomial $M(\{\mathcal{F}\}, T, \Gamma)$ is also a multilinear operator, its homogeneity index $m$ equals $\nu(T)$ and we denote its arguments by $\left(\mathbf{x}_{1} \ldots \mathbf{x}_{m}\right)$. Respectively, if $\mathbf{x}_{1} \ldots \mathbf{x}_{\nu}$ are arguments of a monomial $M(\{\mathcal{F}\}, T, \Gamma)$ and we use the standard labeling of the nodes then according to Proposition 4.14 a submonomial $M(\{\mathcal{F}\}, T, \Gamma)$ has arguments $\mathbf{x}_{\varkappa\left(T^{\prime}\right)}, \ldots, \mathbf{x}_{\varkappa\left(T^{\prime}\right)+\nu\left(T^{\prime}\right)-1}$ which are labeled constructively.

Now we would like to describe elementary properties of composition monomials and the related trees. Note that for every $N \in T$ a composition monomial is a linear function of operator $\mathcal{F}_{\Gamma(N)}^{\mu(N)}$. Consequently, the concept of the decorated composition monomial can be naturally extended to monomials associated with the following family of operators

$$
\{\mathcal{F}\}=\left\{\mathcal{F}: \mathcal{F}=c_{1} \mathcal{F}_{1}+\ldots+c_{l} \mathcal{F}_{l}, c_{i} \in \mathbb{C}\right\} .
$$

For a given tree $T$ the submonomial $M(\{\mathcal{F}\}, \Gamma, T)$ is represented as a function on the tree $T$ with values in $\{\mathcal{F}\}$, this is an $i$-linear function of $\mathcal{F}$ where $i$ is the incidence number of $T$. 
There are elementary relations between the incidence number $i(T)$, the rank $r(T)$, the number of edges of a tree $T$ which do not end at an end node $e^{0}(T)$ and the homogeneity index $m$ of a tree $T$, and corresponding monomial $M(\{\mathcal{F}\}, \Gamma, T)$. For example, $e^{0}(T)=i(T)-1$. Some useful relations expressed by inequalities are given in the following lemma.

Lemma 4.21 Let us consider trees $T$ for which every non-end node has at least two children, $\mu(N) \geq 2$ for all $N \in T$. Let for any $i$ the number $m(i)$ be the minimum number of the end nodes $\nu(T)$ for all trees $T$ with given incidence number $i$. Then

$$
m(i) \geq i+1
$$

Similarly for any given $r$ let $m(r)$ be the minimum number of end nodes with given rank $r$. Then

$$
m(r) \geq r+1 .
$$

Let $e^{0}(T)$ be the number of edges of a tree $T$ which do not end at end nodes. For any given e let $m(e)$ be the minimum number of end nodes with $e^{0}(T)=e$. Then

$$
m\left(e^{0}\right)>e^{0}+1
$$

Proof. For $i=1$ (4.25) is true. Let the statement be true for $i=i_{0}$. Let $T$ be a tree with the minimum number of end nodes $m\left(i_{0}\right)=m$. We delete one of the end nodes together with the edge leading to it from its parent obtaining a tree with $m\left(i_{0}\right)-1$ end node. If the tree remains in the same class, then $m\left(i_{0}\right)$ is reduced by one contradicting the minimality. Hence, the deletion of the edge created a node with only one child. Such a node can be replaced by an edge leading from its parent to its child and reducing the incidence number by one. Using the induction assumption we get

$$
m\left(i_{0}\right)-1 \geq m\left(i_{0}-1\right) \geq\left(i_{0}-1\right)+1
$$

that completes the induction and proves (4.25) for all $i$. Similar induction proves (4.26). For $r=1$ (4.26) is true. Let $T$ be a tree with the minimum number of end nodes $m\left(r_{0}\right)=m$. As above, by deleting an end node and using the minimality we reduce the tree $T$ to a tree $T^{\prime}$ with a smaller rank. Since only one non-end node is eliminated, the rank of $T^{\prime}$ is $r_{0}-1$ and we get (4.26). Inequality (4.27) holds for $e=0$ since $m(0) \geq 2$. Let $T$ be a tree with the minimum number of end nodes $m\left(e_{0}\right)=m$. We again delete one of the end nodes together with the edge joining it to its parent and obtain a tree with $m\left(e_{0}\right)-1$ end nodes and the same number of edges which do not end at an end node. The minimality implies that the parent node has only one another child and removing it we get either $e_{0}$ or $e_{0}-1$ edges which do not go to end nodes. We use the induction as in (4.28) obtaining (4.27).

Monomial expansion in the Implicit Function Theorem If operators $\mathcal{G}^{m}\left(\mathbf{x}_{1} \ldots \mathbf{x}_{m}\right)$ are determined by the recurrent formulas (4.18) it is obvious that every $\mathcal{G}^{m}$ can be represented 
in terms of $\mathcal{F}=\left\{\mathcal{F}^{(s)}\right\}$ using the recurrence and multilinearity of $\mathcal{F}^{(s)}$. More precisely the following representation holds

$$
\mathcal{G}^{(m)}\left(\mathcal{F}, \mathbf{x}_{1} \ldots \mathbf{x}_{m}\right)=\sum_{T \in T_{m}} c_{T} M(\mathcal{F}, T)\left(\mathbf{x}_{1} \ldots \mathbf{x}_{m}\right)
$$

where (i) $M(\mathcal{F}, T) \in \mathcal{T}_{2}$ is a composition monomial corresponding to a tree $T$ and $T_{m} \subset \mathcal{T}_{2}$ stands for the set of trees with $m$ end nodes; (ii) the integer-valued multiplicity coefficient $c_{T} \geq 0$ counts the multiplicity of the related monomial $M(\mathcal{F}, T)$ in the expansion of (4.18); for some trees $T$ its multiplicity coefficient $c_{T}$ may be zero. The expansion (4.29) is obtained by an inductive process with respect to $m$ since (4.18) expresses $\mathcal{G}^{m}$ in terms of $\mathcal{G}^{\left(i_{j}\right)}$ with $2 \leq i_{j}<m$. Notice that for a given operator $\mathcal{F}=\left\{\mathcal{F}^{(s)}\right\}$ the monomial $M(\mathcal{F}, T)$ considered as an operator can be the same for different $T$, the monomials and the multiplicity coefficients are determined purely algebraically.

Remark 4.22 The expression (4.29) for $\mathcal{G}^{(m)}$ as a linear combination of composition monomials $M(\mathcal{F}, T)$, in particular the multiplicity coefficients $c_{T}$, does not depend on a specific form of the operator $\mathcal{F}$. It is the same for a solution $\mathbf{z}=\mathbf{x}+\mathcal{G}(\mathcal{F}, \mathbf{x})$ of the general functional equation (4.1) and for an elementary algebraic equation $u=\mathcal{F}(u)+x$ with $u, x \in \mathbb{C}$ and with a scalar analytic function $\mathcal{F}$ of one complex variable.

If all $\mathcal{F}_{i}^{(m)}$ are bounded multilinear operators then a decorated monomial $M(\mathcal{F}, T, \Gamma)$ is also a bounded multilinear operator as it follows from the following statement.

Lemma 4.23 Let $M(\{\mathcal{F}\}, T, \Gamma)$ be a decorated monomial of the homogeneity index $\nu(T)=$ $m$ and all $\mathcal{F}_{i}^{(s)}$ be bounded operators from $E^{s}$ into $E$ for a Banach space $E$. Then the following estimate holds

$$
\left\|M(\{\mathcal{F}\}, T, \Gamma)\left(\mathbf{x}_{1} \ldots \mathbf{x}_{m}\right)\right\|_{E} \leq \prod_{N \in T, r(N)>0}\left\|\mathcal{F}_{\Gamma(N)}^{(\mu(N))}\right\| \prod_{j=1}^{m}\left\|\mathbf{x}_{j}\right\|_{E} .
$$

Proof. Notice that

$$
\left\|\mathcal{F}^{(m)}\left(M_{1} \ldots M_{m}\right)\right\|_{E} \leq\left\|\mathcal{F}^{(m)}\right\|\left\|M_{1}\right\|_{E} \ldots\left\|M_{m}\right\|_{E}
$$

where $M_{j}$ are submonomials. Applying the above inequality repeatedly we obtain (4.30).

The next statement provides a bound for the norm of a decorated monomial which involves as a factor the norm of a submonomial.

Lemma 4.24 . Let $M(\{\mathcal{F}\}, T, \Gamma)$ be a decorated monomial evaluated at $\mathbf{x}_{1} \ldots \mathbf{x}_{m}$. Let all $\mathcal{F}^{(s)}$ be bounded operators from $E^{s}$ into Banach space $E$. Then for every evaluated submonomial $M\left(\{\mathcal{F}\}, T^{\prime}\left(N_{0}\right), \Gamma\right)$ we have an estimate

$$
\begin{gathered}
\left\|M(\{\mathcal{F}\}, T, \Gamma)\left(\mathbf{x}_{1} \ldots \mathbf{x}_{m}\right)\right\|_{E} \leq \| M\left(\{\mathcal{F}\}, T^{\prime}\left(N_{0}\right), \Gamma\right)\left(\mathbf{x}_{\varkappa}, \ldots, \mathbf{x}_{\left.\varkappa+\nu\left(T^{\prime}(N)\right)-1\right)} \|_{E}\right. \\
\prod_{N \in T \backslash T^{\prime}\left(N_{0}\right), r(N)>0}\left\|\mathcal{F}_{\Gamma(N)}^{(\mu(N))}\right\| \prod_{j<\varkappa}\left\|\mathbf{x}_{j}\right\| \prod_{j \geq \varkappa+\nu\left(T^{\prime}\left(N_{0}\right)\right)}\left\|\mathbf{x}_{j}\right\| .
\end{gathered}
$$

where $\mathbf{x}_{\varkappa}, \ldots, \mathbf{x}_{\varkappa+\nu\left(T^{\prime}(N)\right)-1}$ are the arguments of the submonomial $M\left(\{\mathcal{F}\}, T^{\prime}\left(N_{0}\right), \Gamma\right)$. 
Proof. The proof uses the induction with respect to the length $l\left(N_{0}\right)$. For $l\left(N_{0}\right)=0$ the statement is obvious. Assuming that the statement is true for $l(N)<l_{0}$ we consider the case when $l\left(N_{0}\right)=l_{0}$. Notice that

$$
\left\|\mathcal{F}_{\Gamma\left(N_{*}\right)}^{\left(\mu\left(N_{*}\right)\right)}\left(M_{1} \ldots M_{\mu(N)}\right)\right\|_{E} \leq\left\|\mathcal{F}_{\Gamma\left(N_{*}\right)}^{\left(\mu\left(N_{*}\right)\right)}\right\|\left\|M_{1}\right\|_{E} \ldots\left\|M_{\mu(N)}\right\|_{E}
$$

where $M_{j}=M\left(\{\mathcal{F}\}, T^{\prime}\left(N_{* j}\right), \Gamma\right), N_{* j}$ are child nodes of $N_{*}$. One of the submonomials $M_{1} \ldots M_{\mu(N)}$ contains $M\left(\{\mathcal{F}\}, T^{\prime}\left(N_{0}\right), \Gamma\right)$ as a submonomial, and let it be $M\left(\{\mathcal{F}\}, T^{\prime}\left(N_{* j_{0}}\right), \Gamma\right)$. The length of the path from $N_{0}$ to $N_{* j}$ is less than $l_{0}$ and we can use the induction hypothesis to estimate the norm of $M\left(\{\mathcal{F}\}, T^{\prime}\left(N_{* j_{0}}\right), \Gamma\right)$. The norms of $M_{j}$ with $j \neq j_{0}$ are estimated using (4.30). The labels of the arguments of the submonomial fill an interval according to Proposition 4.14

The following theorem gives a needed refinement of the Implicit Function Theorem 4.7

Theorem 4.25 (refined Implicit Function Theorem) Let $\mathcal{F} \in A\left(C_{\mathcal{F}}, R_{\mathcal{F}}\right)$. Let $\mathcal{G} \in$ $A\left(C_{\mathcal{G}}, R_{\mathcal{G}}\right)$ be the analytic solution operator constructed in Theorem 4.7 which solves $[4.1)$. Then the expansion of $\mathcal{G}(\mathcal{F}, \mathbf{x})$ into composition monomials

$$
\mathcal{G}(\mathcal{F}, \mathbf{x})=\sum_{m=1}^{\infty} \sum_{T \in T_{m}} c_{T} M(\mathcal{F}, T)\left(\mathbf{x}^{m}\right)
$$

converges for $\|\mathbf{x}\|<R_{\mathcal{G}}$, and the following estimates hold

$$
\begin{gathered}
\sum_{T \in T_{m}} c_{T}\left\|M(\mathcal{F}, T)\left(\mathbf{x}^{m}\right)\right\| \leq C_{\mathcal{G}} R_{\mathcal{G}}^{-m}\|\mathbf{x}\|^{m}, m=2, \ldots, \\
\sum_{m=2}^{\infty} \sum_{T \in T_{m}} c_{T}\left\|M(\mathcal{F}, T)\left(\mathbf{x}^{m}\right)\right\| \leq C_{\mathcal{G}} \frac{\|x\|_{X}^{2} R_{\mathcal{G}}^{-2}}{1-\|x\|_{X} R_{\mathcal{G}}^{-1}},
\end{gathered}
$$

where $C_{\mathcal{G}}$ and $R_{\mathcal{G}}$ depend only on $C_{\mathcal{F}}$ and $R_{\mathcal{F}}$ and satisfy

$$
C_{\mathcal{G}}=\frac{R_{\mathcal{F}}^{2}}{2\left(C_{\mathcal{F}}+R_{\mathcal{F}}\right)}, \quad R_{\mathcal{G}}=\frac{R_{\mathcal{F}}^{2}}{4\left(C_{\mathcal{F}}+R_{\mathcal{F}}\right)} .
$$

The multiplicity coefficients $c_{T} \geq 0$ satisfy the inequality

$$
\sum_{T \in T_{m}} c_{T} \leq \frac{1}{4} 8^{m}
$$

The proof of this statement is given in Appendix B. 


\subsection{Decorated expansions}

In this section we develop a formalism for treating linear operators with several invariant subspaces which span the entire space as, for example, in the case of projections (2.19). The decomposition into related invariant subspaces is very important for the analysis. The general setting is as follows. Suppose that a Banach space $E$ has several projection operators $\Pi_{\lambda}, \lambda \in \Lambda$, where $\Lambda$ is a finite set of indices, we call this set decoration set. We assume that the sum of the projections equals the identical operator, i.e.

$$
\sum_{\lambda \in \Lambda} \Pi_{\lambda}=\mathrm{Id}, \text { where Id is the identity operator, }
$$

and

$$
\Pi_{\lambda} \Pi_{\lambda}=\Pi_{\lambda}, \Pi_{\lambda^{\prime}} \Pi_{\lambda}=0 \text { if } \lambda^{\prime} \neq \lambda, \lambda^{\prime}, \lambda \in \Lambda
$$

We call such projections decoration projections. For example, let us look at projections $\Pi_{n, \zeta}(\mathbf{k}), n=1, \ldots, J, \zeta= \pm$ defined by (2.19). These projections define bounded operators $\Pi_{n, \zeta}$ acting on (i) functions of $\mathbf{k}$ in the space $L_{1}$; (ii) functions of $\mathbf{k}, \tau$ in the space $E=$ $C\left(\left[0, \tau_{*}\right], L_{1}\right)$. In another example based on (2.19) we fix $n_{0}$ and define

$$
\Pi_{\zeta}(\mathbf{k})=\Pi_{n_{0}, \zeta}(\mathbf{k}), \zeta= \pm, \Pi_{\infty}(\mathbf{k})=\sum_{n \neq n_{0}, \zeta= \pm} \Pi_{n, \zeta}(\mathbf{k})
$$

Using (4.36) we expand vectors $\mathrm{x} \in E$ as follows

$$
\mathbf{x}=\sum_{\lambda \in \Lambda} \Pi_{\lambda} \mathbf{x}=\sum_{\lambda \in \Lambda} \mathbf{x}_{\lambda}, \mathbf{x}_{\lambda}=\Pi_{\lambda}(\mathbf{x})
$$

We also use notation

$$
\mathcal{F}_{\lambda}^{(n)}=\Pi_{\lambda} \mathcal{F}^{(n)}
$$

Often in applications the number of elements in $\Lambda$ is either 2 or 3 . In the case when $\Lambda$ has three elements we set

$$
\Lambda=\{+,-, \infty\}, \Pi_{+}+\Pi_{-}+\Pi_{\infty}=\mathrm{Id},
$$

and

$$
\mathbf{x}=\mathbf{x}_{+}+\mathbf{x}_{-}+\mathbf{x}_{\infty}, \mathcal{F}(\mathbf{x})=\mathcal{F}_{+}(\mathbf{x})+\mathcal{F}_{-}(\mathbf{x})+\mathcal{F}_{\infty}(\mathbf{x}) .
$$

Using the decomposition (4.36) we introduce for $m$-linear operators $\mathcal{F}^{(n)}\left(\mathbf{x}_{1} \ldots \mathbf{x}_{n}\right)$ the corresponding decorated operators $\mathcal{F}_{\lambda, \vec{\zeta}}^{(n)}$ as follows:

$$
\mathcal{F}_{\lambda, \vec{\zeta}}^{(n)}\left(\mathbf{x}_{1} \ldots \mathbf{x}_{n}\right)=\Pi_{\lambda} \mathcal{F}^{(n)}\left(\Pi_{\zeta^{\prime}} \mathbf{x}_{1} \ldots \Pi_{\zeta^{(n)}} \mathbf{x}_{n}\right)=\mathcal{F}_{\lambda}^{(n)}\left(\Pi_{\zeta^{\prime}} \mathbf{x}_{1} \ldots \Pi_{\zeta^{(n)}} \mathbf{x}_{n}\right)
$$

where $\vec{\zeta}$ is defined in (3.7). Obviously, we have

$$
\mathcal{F}^{(n)}\left(\mathbf{x}_{1} \ldots \mathbf{x}_{n}\right)=\sum_{\lambda \in \Lambda, \vec{\zeta} \in \Lambda^{n}} \mathcal{F}_{\lambda, \vec{\zeta}}^{(n)}\left(\mathbf{x}_{1} \ldots \mathbf{x}_{n}\right) .
$$

An example of expansion (4.44) is given by (3.11). 


\subsection{Decorated composition monomials}

We assume that operators $\mathcal{F}^{(n)}$ act in the space allowing a decomposition into three components as in (4.41). Let $M(\mathcal{F}, T)$ be a composition monomial of the homogeneity index $m$, and assume that the corresponding tree $T$ has the incidence number $i$, the rank $r$, and $e$ edges. Suppose also that every operator $\mathcal{F}^{(n)}$ is expanded into a sum of decorated operators as in (4.44), (4.43). Using the linearity of $M(\mathcal{F}, T)$ with respect to operators $\mathcal{F}^{(n)}$ we get

$$
\begin{gathered}
M(\mathcal{F}, T)=\mathcal{F}^{(n)}\left(\mathcal{F}^{\left(m_{1}\right)}(\ldots) \ldots \mathcal{F}^{\left(m_{n}\right)}(\ldots)\right)= \\
\sum_{\lambda \in \Lambda, \vec{\lambda} \in \Lambda^{i-1}, \vec{\zeta}_{j}, j=1, \ldots, e} \mathcal{F}_{\lambda}^{(n)}\left(\mathcal{F}_{\lambda_{j_{1}}, \vec{\zeta}_{j_{1}}}^{\left(m_{1}\right)}(\ldots) \ldots \mathcal{F}_{\lambda_{j_{n}}, \vec{\zeta}_{j_{n}}}^{\left(m_{n}\right)}(\ldots)\right),
\end{gathered}
$$

where submonomials $\mathcal{F}_{\lambda_{1}, \vec{\zeta}_{1}}^{\left(m_{1}\right)}(\ldots), \ldots, \mathcal{F}_{\lambda_{n}, \vec{\zeta}_{n}}^{\left(m_{n}\right)}(\ldots)$ have ranks not exceeding $r-1$. We expanded repeatedly the expression in the left-hand side of (4.45) as long as submonomials of non-zero rank were present resulting in an expansion involving only decorated operators $\mathcal{F}_{\lambda, \vec{\zeta}}^{(n)}$.

Remark 4.26 Note that

$$
\mathcal{F}_{\lambda}^{(n)}\left(\mathcal{F}_{\lambda_{1}, \vec{\zeta}_{1}}^{\left(m_{1}\right)}(\ldots) \ldots \mathcal{F}_{\lambda_{n}, \vec{\zeta}_{n}}^{\left(m_{n}\right)}(\ldots)\right)=\mathcal{F}_{\lambda}^{(n)}\left(\Pi_{\lambda_{1}} \mathcal{F}_{\lambda_{1}, \vec{\zeta}_{1}}^{\left(m_{1}\right)}(\ldots) \ldots \Pi_{\lambda_{n}} \mathcal{F}_{\lambda_{n}, \vec{\zeta}_{n}}^{\left(m_{n}\right)}(\ldots)\right)
$$

Since projections $\Pi_{\zeta}$ satisfy the identities 4.37$)$ if a vector $\vec{\zeta}=\left(\zeta^{\prime}, \ldots, \zeta^{(n)}\right)$ and indices $\lambda_{1}, \ldots, \lambda_{n}$ are given, then we have the identity

$$
\mathcal{F}_{\lambda, \vec{\zeta}}^{(n)}\left(\mathcal{F}_{\lambda_{1}, \vec{\zeta}_{1}}^{\left(m_{1}\right)} \ldots \mathcal{F}_{\lambda_{n}, \vec{\zeta}_{n}}^{\left(m_{n}\right)}\right)=0 \text { when } \lambda_{i} \neq \zeta^{(i)} \text { for some } i
$$

Hence, for non-zero terms in the expansion 4.45) if indices $\lambda_{1}, \ldots, \lambda_{n}$ for $\mathcal{F}_{\lambda_{1}, \vec{\zeta}_{1}}^{\left(m_{1}\right)}, \ldots, \mathcal{F}_{\lambda_{n}, \vec{\zeta}_{n}}^{\left(m_{n}\right)}$ are given the vector $\vec{\zeta}$ in $\mathcal{F}_{\lambda, \vec{\zeta}}^{(n)}$ is determined by them

$$
\zeta^{(i)}=\lambda_{i}, i=1, \ldots, n
$$

Note that according to (4.47) and (4.48) we have

$$
\mathcal{F}_{\lambda, \vec{\lambda}}^{(n)}\left(\mathcal{F}^{\left(m_{1}\right)}(\ldots) \ldots \mathcal{F}^{\left(m_{n}\right)}(\ldots)\right)=\mathcal{F}_{\lambda}^{(n)}\left(\mathcal{F}_{\lambda_{1}}^{\left(m_{1}\right)}(\ldots) \ldots \mathcal{F}_{\lambda_{n}}^{\left(m_{n}\right)}(\ldots)\right)
$$

According to (4.45), (4.49) for every tree $T$ of the homogeneity index $m$ and the incidence number $i$, we get an expansion into a sum of monomials of the form

$$
\begin{aligned}
& M(\mathcal{F}, T, \vec{\lambda}, \vec{\zeta})\left(\mathbf{x}_{1} \mathbf{x}_{2} \ldots \mathbf{x}_{m}\right)=M(\{\mathcal{F}\}, \Gamma, T)\left(\mathbf{x}_{1} \mathbf{x}_{2} \ldots \mathbf{x}_{m}\right) \\
& \{\mathcal{F}\}=\left\{\mathcal{F}_{\lambda, \vec{\zeta}}^{(n)}: \lambda \in \Lambda, \vec{\zeta} \in \Lambda^{n}, n=2,3, \ldots\right\} .
\end{aligned}
$$


Namely, if a monomial $M(\mathcal{F}, T)$ has at a node $N$ operator $\mathcal{F}^{(m(N))}$ then $M(\{\mathcal{F}\}, \Gamma, T)$ at this node has operator $\mathcal{F}_{\Gamma(N)}^{(m(N))}$. We call a composition monomial of the form (4.50), where (4.48) is assumed, a decorated composition monomial. Using the standard labeling of nodes, for a given function $\Gamma$ on the tree $T$ with values in $\Lambda$ we find the vectors $\vec{\lambda} \in \Lambda^{i}, \vec{\zeta} \in \Lambda^{m}$, with $i$ being the incidence number of the tree $T$, and using (4.48) we rewrite (4.45) in the form

$$
M(\mathcal{F}, T)\left(\mathbf{x}_{1} \mathbf{x}_{2} \ldots \mathbf{x}_{m}\right)=\sum_{\vec{\lambda} \in \Lambda^{i}, \vec{\zeta} \in \Lambda^{m}} M(\mathcal{F}, T, \vec{\lambda}, \vec{\zeta})\left(\mathbf{x}_{1} \mathbf{x}_{2} \ldots \mathbf{x}_{m}\right) .
$$

where $\vec{\zeta}$ is determined by values of $\Gamma$ on the end nodes. The sum (4.51) contains at most $3^{i+m}$ non-zero terms, where 3 is the number of elements in $\Lambda$. Combining (4.51) with (4.33) we obtain

$$
\mathcal{G}^{(m)}\left(\mathbf{x}^{m}\right)=\sum_{T \in T_{m}} \sum_{\vec{\lambda} \in \Lambda^{i(T)}, \vec{\zeta} \in \Lambda^{m}} c_{T} M(\mathcal{F}, T, \vec{\lambda}, \vec{\zeta})\left(\mathbf{x}^{m}\right) .
$$

\section{Expansions of solutions for oscillatory integral equa- tion}

In this section we apply general concepts introduced in previous sections to oscillatory integrals involving operators $\mathcal{F}$ as in (3.3), (3.4). Based on projections $\Pi_{n, \zeta}(\mathbf{k})$ in (2.19) for given $n=n_{0}$ we define as in (4.38) decoration projections in $L_{1}$ which satisfy (4.41):

$$
\Pi_{\zeta} \tilde{\mathbf{u}}(\mathbf{k})=\Pi_{n_{0}, \zeta}(\mathbf{k}) \tilde{\mathbf{u}}(\mathbf{k}), \zeta= \pm, \Pi_{\infty}=\sum_{n \neq n_{0}} \sum_{\zeta= \pm} \Pi_{n, \zeta}
$$

\subsection{Boundedness of oscillatory integral operators}

In this subsection we estimate norms of multilinear operators $\mathcal{F}=\mathcal{F}^{(m)}$ defined by (3.4) and the related composition monomials. The operators $\mathcal{F}^{(m)}$ have the form (3.4) where $\mathbb{D}_{m}=\mathbb{R}^{d(m-1)}$ as in (2.65) or $\mathbb{D}_{m}=[-\pi, \pi]^{d(m-1)}$ as in (2.23). The both cases are completely similar since we use the same properties of the spaces $L_{1}=L_{1}\left([-\pi, \pi]^{d}\right)$ or $L_{1}=L_{1}\left(\mathbb{R}^{d}\right)$, and we do not use in our proofs the boundedness and compactness of the domain $[-\pi, \pi]^{d}$. Hence, we will everywhere consider the periodic case $[-\pi, \pi]^{d}$ which corresponds to lattice equations and without further comment apply the results to the case $\mathbb{R}^{d}$.

Lemma 5.1 The operator $\mathcal{F}^{(m)}$ defined by (3.4), (2.22) is bounded from $E=C\left(\left[0, \tau_{*}\right], L_{1}\right)$ into $C^{1}\left(\left[0, \tau_{*}\right], L_{1}\right)$ and its norm is estimated as follows

$$
\begin{aligned}
& \left\|\mathcal{F}^{(m)}\left(\tilde{\mathbf{u}}_{1} \ldots \tilde{\mathbf{u}}_{m}\right)\right\|_{E} \leq \tau_{*} C_{\Xi}^{2 m+1}\left\|\chi^{(m)}\right\| \prod_{j=1}^{m}\left\|\tilde{\mathbf{u}}_{j}\right\|_{E} \\
& \left\|\partial_{\tau} \mathcal{F}^{(m)}\left(\tilde{\mathbf{u}}_{1} \ldots \tilde{\mathbf{u}}_{m}\right)\right\|_{E} \leq C_{\Xi}^{2 m+1}\left\|\chi^{(m)}\right\| \prod_{j}\left\|\tilde{\mathbf{u}}_{j}\right\|_{E} .
\end{aligned}
$$


Proof. According to Condition 2.5 we can diagonalize the matrix $\exp \left\{-\mathrm{i} \mathbf{L}(\mathbf{k}) \frac{\tau_{1}}{\varrho}\right\}$ and its norm is bounded uniformly in $\mathbf{k}, \tau_{1}$ and $\varrho$ :

$$
\left\|\exp \left\{-\mathrm{i} \mathbf{L}(\mathbf{k}) \frac{\tau_{1}}{\varrho}\right\}\right\| \leq C_{\Xi}^{2} \forall \mathbf{k} \in \mathbb{R}^{d}, \varrho>0, \tau_{1} \geq 0 .
$$

By (3.4), (3.5) and (2.22)

$$
\begin{gathered}
\left\|\mathcal{F}^{(m)}\left(\tilde{\mathbf{u}}_{1} \ldots \tilde{\mathbf{u}}_{m}\right)(\cdot, \tau)\right\|_{L_{1}} \leq C_{\Xi}^{2 m+1} \sup _{\mathbf{k}, \vec{k}}\left|\chi^{(m)}(\mathbf{k}, \vec{k})\right| \iint_{0}^{\tau} \int_{\mathbb{D}_{m}} \\
\left|\tilde{\mathbf{u}}_{1}\left(\mathbf{k}^{\prime}\right)\right| \ldots\left|\tilde{\mathbf{u}}_{m}\left(\mathbf{k}^{(m)}(\mathbf{k}, \vec{k})\right)\right| \mathrm{d} \mathbf{k}^{\prime} \ldots \mathrm{d} \mathbf{k}^{(m-1)} \mathrm{d} \tau_{1} \mathrm{~d} \mathbf{k} \leq \\
C_{\Xi}^{2 m+1}\left\|\chi^{(m)}\right\| \int_{0}^{\tau}\left\|\tilde{\mathbf{u}}_{1}\left(\tau_{1}\right)\right\|_{L_{1}} \ldots\left\|\tilde{\mathbf{u}}_{m}\left(\tau_{1}\right)\right\|_{L_{1}} \mathrm{~d} \tau_{1} \leq \tau_{*} C_{\Xi}^{2 m+1}\left\|\chi^{(m)}\right\|\left\|\tilde{\mathbf{u}}_{1}\right\|_{E} \ldots\left\|\tilde{\mathbf{u}}_{m}\right\|_{E} .
\end{gathered}
$$

Similarly,

$$
\begin{gathered}
\left\|\partial_{\tau} \mathcal{F}^{(m)}\left(\tilde{\mathbf{u}}_{1} \ldots \tilde{\mathbf{u}}_{m}\right)(\cdot, \tau)\right\|_{L_{1}} \leq \\
C_{\Xi}^{2 m+1}\left\|\chi^{(m)}\right\| \iint_{\mathbb{D}_{m}}\left|\tilde{\mathbf{u}}_{1}\left(\mathbf{k}^{\prime}\right)\right| \ldots\left|\tilde{\mathbf{u}}_{m}\left(\mathbf{k}^{(m)}(\mathbf{k}, \vec{k})\right)\right| \mathrm{d} \mathbf{k}^{\prime} \ldots \mathrm{d} \mathbf{k}^{(m-1)} \mathrm{d} \mathbf{k} \leq \\
\left\|\chi^{(m)}\right\|\left\|\tilde{\mathbf{u}}_{1}\right\|_{E} \ldots\left\|\tilde{\mathbf{u}}_{m}\right\|_{E} \cdot
\end{gathered}
$$

Corollary 5.2 If $M(\mathcal{F}, T, \vec{\lambda}, \vec{\zeta})\left(\mathbf{x}_{1} \ldots \mathbf{x}_{m}\right)$ is a decorated composition monomial defined by (4.18) and $\mathcal{F}$ is defined by (3.3), 3.4 then

$$
\begin{gathered}
\left\|M(\mathcal{F}, T, \vec{\lambda}, \vec{\zeta})\left(\mathbf{x}_{1} \ldots \mathbf{x}_{m}\right)\right\|_{E} \leq C_{\Xi}^{2 e+i} \tau_{*}^{i} \prod_{N \in T}\left\|\chi^{(\mu(N))}\right\| \prod_{l=1}^{m}\left\|\mathbf{x}_{l}\right\|_{E}, \\
\left\|\partial_{\tau} M(\mathcal{F}, T, \vec{\lambda}, \vec{\zeta})\left(\mathbf{x}_{1} \ldots \mathbf{x}_{m}\right)\right\|_{E} \leq C_{\Xi}^{2 e+i} \tau_{*}^{i-1} \prod_{N \in T}\left\|\chi^{(\mu(N))}\right\| \prod_{l=1}^{m}\left\|\mathbf{x}_{l}\right\|_{E}
\end{gathered}
$$

where $i$ is the incidence number of the tree $T$, and $e$ is the number of edges of $T$.

Proof. We estimate the norm of the monomial $M=\mathcal{F}^{(m)}\left(M_{1} \ldots M_{m}\right)$ and its time derivative applying Lemma 5.1]. Then we use (5.2) to estimate $\left\|M_{j}\right\|_{C\left(\left[0, \tau_{*}\right], L_{1}\right)}$. The formal proof is straightforward and uses the induction with respect to the incidence number of a monomial.

Using boundedness of operators $\mathcal{F}^{(m)}$ we obtain in a standard way uniqueness of solution of (3.3).

Lemma 5.3 If $\tilde{\mathbf{u}}_{1}, \tilde{\mathbf{u}}_{2} \in C\left(\left[0, \tau_{0}\right], L_{1}\right)$ with $\tau_{0}>0$ are two solutions of (3.3) with the same $\tilde{\mathbf{h}}$ then $\tilde{\mathbf{u}}_{1}=\tilde{\mathbf{u}}_{2}$. 
Proof. Applying Lemma 4.6, we conclude that

$$
\left\|\mathcal{F}\left(\tilde{\mathbf{u}}_{1}\right)-\mathcal{F}\left(\tilde{\mathbf{u}}_{2}\right)\right\|_{C\left(\left[0, \tau_{1}\right], L_{1}\right)} \leq C \tau_{1}\left\|\mathcal{F}\left(\tilde{\mathbf{u}}_{1}\right)-\mathcal{F}\left(\tilde{\mathbf{u}}_{2}\right)\right\|_{C\left(\left[0, \tau_{1}\right], L_{1}\right)}, 0<\tau_{1} \leq \tau_{0}
$$

Deriving the above inequality we use that since $N_{F}<\infty$ the radius $R_{\mathcal{F}}$ in Lemma 4.6] is arbitrary large and $C_{\mathcal{F}}$ in (4.13) according to (5.2) is proportional to $\tau_{1}$. When the Lipschitz constant $C \tau_{1}<1$, in a standard way we obtain that $\tilde{\mathbf{u}}_{1}(\tau)=\tilde{\mathbf{u}}_{2}(\tau)$ for $0 \leq \tau \leq \tau_{1}$. Since this statement can be applied to $\tilde{\mathbf{u}}_{1}\left(\tau-\tau_{1}\right)$ and $\tilde{\mathbf{u}}_{2}\left(\tau-\tau_{1}\right)$ we obtain that solutions coincide for $0 \leq \tau \leq \tau_{0}$.

\subsection{Function-analytic expansion of solutions for modal integral evolution equation}

The reduced evolution equation (3.3) has the form

$$
\tilde{\mathbf{u}}=\mathcal{F}(\tilde{\mathbf{u}})+\tilde{\mathbf{x}}
$$

where $\tilde{\mathbf{u}}, \tilde{\mathbf{x}}$ are functions of $(\mathbf{k}, \tau)$. The nonlinear operator $\mathcal{F}$ in the right-hand side of (5.7) is determined by (3.4), $\tilde{\mathbf{x}}(\mathbf{k}, \tau)=\tilde{\mathbf{h}}(\mathbf{k})$ as in (3.3). We look for the solution operator $\mathcal{G}$ in the form of operator series

$$
\tilde{\mathbf{u}}=\mathcal{G}(\tilde{\mathbf{x}})=\sum_{m=1}^{\infty} \mathcal{G}^{(m)}\left(\tilde{\mathbf{x}}^{(m)}\right)
$$

The questions related to the existence and the convergence of such series are addressed in Theorem 4.7. As a direct corollary of Theorem 4.7 and Lemma 5.3 if applied to the reduced evolution equation (3.3) we obtain the following theorem.

Theorem 5.4 Let

$$
\|\tilde{\mathbf{x}}\|_{E}<R_{\mathcal{G}}=\left(\tau_{*} C_{\chi} C_{\Xi}^{2 m_{F}+1}\right)^{-1 /\left(m_{F}-1\right)} / 8, \tau_{*} \leq C_{\Xi}^{-3} C_{\chi}^{-1} .
$$

with $C_{\chi}$ as in (2.26), $C_{\Xi}$ as in 2.17). Then the series (5.8) converges in $E=C\left(\left[0, \tau_{*}\right], L_{1}\right)$. The solution operator $\mathcal{G}(\tilde{\mathbf{x}})=\tilde{\mathbf{u}}$ determines the solution to 5.7$)$ and the operators $\mathcal{G}^{(m)}$ in series (5.8) satisfy the recursive relations (4.18).

Proof. ¿From (2.26) and (15.2) we infer that $\mathcal{F}$ defined by (2.21) belongs to the class $A\left(C_{\mathcal{F}}, R_{\mathcal{F}}\right)$ if

$$
\tau_{*} C_{\chi} C_{\Xi}^{2 m+1} \leq C_{\mathcal{F}} R_{\mathcal{F}}^{-m}, m=2, \ldots, m_{F} .
$$

If $C_{\Xi}^{-2} R_{\mathcal{F}}^{-1} \leq 1$ it is sufficient to verify the above condition at $m=m_{F}$ only. After this we apply Theorem 4.7 where according to (4.19) we can take

$$
C_{\mathcal{G}}=\frac{R_{\mathcal{F}}^{2}}{2\left(C_{\mathcal{F}}+R_{\mathcal{F}}\right)}, R_{\mathcal{G}}=\frac{R_{\mathcal{F}}^{2}}{4\left(C_{\mathcal{F}}+R_{\mathcal{F}}\right)}
$$


We take

$$
C_{\mathcal{F}}=R_{\mathcal{F}}=\left(\tau_{*} C_{\chi} C_{\Xi}^{2 m_{F}+1}\right)^{-1 /\left(m_{F}-1\right)}, C_{\mathcal{G}}=2 R_{\mathcal{G}}=R_{\mathcal{F}} / 4
$$

and apply Theorem 4.7. Note that $C_{\Xi}^{-2} R_{\mathcal{F}}^{-1} \leq 1$ if $\tau_{*} \leq C_{\Xi}^{-3} C_{\chi}^{-1}$.

¿From Theorem 5.4 (observing that by (15.11) $R_{\mathcal{F}} \rightarrow \infty$ when $\tau_{*} \rightarrow 0$ ) we obtain Theorems 2.8 and Theorem 2.18.

To prove Theorem 2.15] on the superposition principle we apply the solution operator $\mathcal{G}$ to a sum of wavepackets $\tilde{\mathbf{h}}_{l}(\mathbf{k}, \beta)$ as in Definition 2.9. For technical reasons we have to modify the wavepackets using cut-off functions described below.

Cutoff functions. We often use an infinitely smooth cutoff function $\Psi(\boldsymbol{\eta}), \boldsymbol{\eta} \in \mathbb{R}^{d}$, satisfying the following relations

$$
\begin{aligned}
0 & \leq \Psi(\boldsymbol{\eta}) \leq 1, \Psi(-\boldsymbol{\eta})=\Psi(\boldsymbol{\eta}), \\
\Psi(\boldsymbol{\eta}) & =1 \text { for }|\boldsymbol{\eta}| \leq \pi_{0} / 2, \Psi(\boldsymbol{\eta})=0 \text { for }|\boldsymbol{\eta}| \geq \pi_{0},
\end{aligned}
$$

where $\pi_{0} \leq 1$ is a sufficiently small number which satisfies the inequality

$$
0<\pi_{0}<\frac{1}{2} \min _{l} \operatorname{dist}\left\{\mathbf{k}_{* l}, \sigma\right\} .
$$

Using $\Psi$ we introduce cutoff functions $\Psi_{l, \zeta}(\mathbf{k}, \beta)$ with support near $\zeta \mathbf{k}_{* l}$ defined as follows:

$$
\Psi_{l, \zeta}(\mathbf{k}, \beta)=\Psi\left(\frac{\mathbf{k}-\zeta \mathbf{k}_{* l}}{\beta^{1-\epsilon}}\right), l=1, \ldots, N_{h} .
$$

Here $\epsilon$ is a small number, $1 / 2>\epsilon>0$; we take the same $\epsilon$ as in Definition 2.9.

Given a wavepacket $\tilde{\mathbf{h}}_{l}(\mathbf{k}, \beta)$ we introduce a modified wavepacket

$$
\tilde{\mathbf{h}}_{l}^{\Psi}(\mathbf{k}, \beta)=\tilde{\mathbf{h}}_{l,+}^{\Psi}(\mathbf{k}, \beta)+\tilde{\mathbf{h}}_{l,-}^{\Psi}(\mathbf{k}, \beta), \tilde{\mathbf{h}}_{l, \zeta}^{\Psi}(\mathbf{k}, \beta)=\Psi_{l, \zeta}(\mathbf{k}, \beta) \tilde{\mathbf{h}}_{l, \zeta}(\mathbf{k}, \beta),
$$

where $\Psi_{l, \zeta}$ are defined by (5.14).

Proposition 5.5 If $\tilde{\mathbf{h}}_{l}(\mathbf{k}, \beta)$ is a wavepacket in the sense of Definition 2.9 then $\tilde{\mathbf{h}}_{l}^{\Psi}(\mathbf{k}, \beta)$ defined by (5.15) and (5.14) is also a wavepacket in the sense of Definition [2.9 and, in addition to that,

$$
\begin{gathered}
\tilde{\mathbf{h}}_{l, \zeta}^{\Psi}(\mathbf{k}, \beta)=0 \text { if }\left|\mathbf{k}-\zeta \mathbf{k}_{* l}\right| \geq \pi_{0} \beta^{1-\epsilon}, \\
\left\|\tilde{\mathbf{h}}_{l}-\tilde{\mathbf{h}}_{l}^{\Psi}\right\|_{L_{1}} \leq C \beta .
\end{gathered}
$$

Proof. To obtain (5.17) we note that (2.34) and (5.12) imply:

$$
\left\|\left(1-\Psi_{l, \zeta}\right) \tilde{\mathbf{h}}_{l, \zeta}\right\|_{L_{1}}=\int\left|\left(1-\Psi_{l, \zeta}(\mathbf{k}, \beta)\right) \tilde{h}_{l, \zeta}(\mathbf{k})\right| \mathrm{d} \mathbf{k} \leq C \beta,
$$

and (5.17) follows. Remaining statements are obtained by a straightforward verification.

The following lemma shows that we can replace $\tilde{\mathbf{h}}_{l}$ by $\tilde{\mathbf{h}}_{l}^{\Psi}$ in the statement of Theorem 2.15, in particular in (2.47), (2.48). 
Lemma 5.6 Let $\tilde{\mathbf{h}}_{l, \zeta}$ satisfy 2.34) and $\tilde{\mathbf{h}}_{l}^{\Psi}(\mathbf{k}, \beta)$ be defined by (5.15). Let

$$
\left\|\tilde{\mathbf{h}}_{l}\right\| \leq R, l=1, \ldots, N_{h} \text { where } N_{h} R<R_{\mathcal{G}}
$$

Then the difference

$$
\left[\mathcal{G}\left(\sum_{l=1}^{N_{h}} \tilde{\mathbf{h}}_{l}\right)-\sum_{l=1}^{N_{h}} \mathcal{G}\left(\tilde{\mathbf{h}}_{l}\right)\right]-\left[\mathcal{G}\left(\sum_{l=1}^{N_{h}} \tilde{\mathbf{h}}_{l}^{\Psi}\right)-\sum_{l=1}^{N_{h}} \mathcal{G}\left(\tilde{\mathbf{h}}_{l}^{\Psi}\right)\right]=B_{\Psi}
$$

is small, namely

$$
\left\|B_{\Psi}\right\|_{E} \leq C(R) \beta
$$

Proof. Note that since $0 \leq \Psi_{l} \leq 1$ we have

$$
\left\|\Psi_{l, \zeta} \tilde{\mathbf{h}}_{l, \zeta}\right\|_{L_{1}} \leq\left\|\tilde{\mathbf{h}}_{l, \zeta}\right\|_{L_{1}},\left\|\left(1-\Psi_{l, \zeta}\right) \tilde{\mathbf{h}}_{l, \zeta}\right\|_{L_{1}} \leq\left\|\tilde{\mathbf{h}}_{l, \zeta}\right\|_{L_{1}},
$$

and (5.18). Using the Lipschitz continuity of the solution operator $\mathcal{G}$ (see 4.6) and (5.17) we obtain (5.21).

Truncation. We will truncate the infinite series (5.8). To this end we define an integer $m=m\left(\beta^{q}\right)$ as a solution of the inequality

$$
\frac{2\left|\ln \beta^{q}\right|}{\left|\ln R_{\mathcal{G}}\right|}<m\left(\beta^{q}\right) \leq \frac{2\left|\ln \beta^{q}\right|}{\left|\ln R_{\mathcal{G}}\right|}+1
$$

where $R_{\mathcal{G}}$ is the same as in (5.9). We consider then the following partial sum of the expansion (5.8)

$$
\mathcal{G}_{m\left(\beta^{q}\right)}(\tilde{\mathbf{h}})=\sum_{m=1}^{m\left(\beta^{q}\right)} \mathcal{G}^{(m)}\left(\tilde{\mathbf{h}}^{(m)}\right)
$$

and readily conclude that the following statement holds.

Lemma 5.7 Let $\mathcal{G}$ be defined by (5.8), then

$$
\left\|\mathcal{G}(\tilde{\mathbf{h}})-\mathcal{G}_{m(\beta)}(\tilde{\mathbf{h}})\right\|_{E} \leq C(R) \beta \text { when }\|\tilde{\mathbf{h}}\|_{E} \leq R<R_{\mathcal{G}} .
$$

\subsubsection{SI-CI splitting for evaluated monomials}

We consider a function $\tilde{\mathbf{h}}$ which is a sum of the form (2.39) and the solution $\mathcal{G}(\mathcal{F}, \tilde{\mathbf{h}})$. Expanding $\mathcal{G}^{(m)}\left(\tilde{\mathbf{h}}^{(m)}\right)$ into composition monomials as in (4.33) we obtain a sum of composition monomials $M(\mathcal{F}, T)\left(\tilde{\mathbf{h}}^{m}\right)$. Then we look at the $m$-linear monomial $M(\mathcal{F}, T)\left(\tilde{\mathbf{h}}^{m}\right)$ where $\tilde{\mathbf{h}}$ 
equals a sum of $N_{h}$ one-band wavepacket $\tilde{\mathbf{h}}_{l}$ as in (2.39). Using the linearity with respect to each argument we expand the monomial into a sum of $N_{h}^{m}$ expressions (evaluated monomials)

$$
M(\mathcal{F}, T)\left(\sum_{l=1}^{N_{h}} \tilde{\mathbf{h}}_{l}\right)^{m}=\sum_{l_{1}, \ldots, l_{m}} M(\mathcal{F}, T)\left(\tilde{\mathbf{h}}_{l_{1}} \ldots \tilde{\mathbf{h}}_{l_{m}}\right)=\sum_{l_{1}, \ldots, l_{m}} M(\mathcal{F}, T)\left(\prod_{i} \tilde{\mathbf{h}}_{l_{i}}\right) .
$$

The sum contains evaluated monomials of two kinds: (i) ones which involve the same wavepacket; and (ii) one corresponding to the cross-terms (terms involving different wavepackets). To be precise, we introduce the following definition.

Definition 5.8 (SI and CI) We say that an evaluated monomial $M(\mathcal{F}, T)\left(\tilde{\mathbf{h}}_{l_{1}} \ldots \tilde{\mathbf{h}}_{l_{m}}\right)$ with the argument multiindex $l_{1}, \ldots, l_{m} \in\{1, \ldots, N\}^{m}$ in the expansion (5.26) is self-interacting (SI) if

$$
l_{1}=l_{2}=\ldots=l_{m}
$$

Otherwise we say that $M(\mathcal{F}, T)\left(\tilde{\mathbf{h}}_{l_{1}} \ldots \tilde{\mathbf{h}}_{l_{m}}\right)$ is cross-interacting $(C I)$.

Using this notation we rewrite (5.26):

$$
M(\mathcal{F}, T)\left(\left(\sum_{l=1}^{N_{h}} \tilde{\mathbf{h}}_{l}\right)^{m}\right)=\sum_{l=1}^{N_{h}} M(\mathcal{F}, T)\left(\left(\tilde{\mathbf{h}}_{l}\right)^{m}\right)+\sum_{l_{1}, \ldots, l_{m} \text { is CI }} M(\mathcal{F}, T)\left(\tilde{\mathbf{h}}_{l_{1}} \ldots \tilde{\mathbf{h}}_{l_{m}}\right) .
$$

Substituting this expression into (4.33) we obtain the expansion

$$
\begin{gathered}
\mathcal{G}\left(\tilde{\mathbf{h}}_{1}+\ldots+\tilde{\mathbf{h}}_{N_{h}}\right)=\sum_{m=1}^{\infty} \mathcal{G}_{m}\left(\left(\tilde{\mathbf{h}}_{1}+\ldots+\tilde{\mathbf{h}}_{N_{h}}\right)^{m}\right) \\
=\sum_{m=1}^{\infty} \mathcal{G}\left(\left(\tilde{\mathbf{h}}_{1}\right)^{m}\right)+\ldots+\sum_{m=1}^{\infty} \mathcal{G}\left(\left(\tilde{\mathbf{h}}_{N_{h}}\right)^{m}\right)+\mathcal{G}_{\mathrm{CI}}\left(\tilde{\mathbf{h}}_{1}, \ldots, \tilde{\mathbf{h}}_{N_{h}}\right),
\end{gathered}
$$

where $\mathcal{G}_{\mathrm{CI}}$ contains only CI monomials with cross-terms.

Proposition 5.9 Every evaluated CI monomial $M(\mathcal{F}, T)\left(\tilde{\mathbf{h}}_{1}, \ldots, \tilde{\mathbf{h}}_{N_{h}}\right)$ has a submonomial of the form

$$
\mathcal{F}^{(s)}\left(M\left(\mathcal{F}, T_{1}\right)\left(\tilde{\mathbf{h}}_{l_{1}} \ldots \tilde{\mathbf{h}}_{l_{1}}\right) \ldots M\left(\mathcal{F}, T_{s}\right)\left(\tilde{\mathbf{h}}_{l_{s}} \ldots \tilde{\mathbf{h}}_{l_{s}}\right)\right)
$$

where all $M\left(\mathcal{F}, T_{1}\right)\left(\tilde{\mathbf{h}}_{l_{1}} \ldots \tilde{\mathbf{h}}_{l_{1}}\right), \ldots, M\left(\mathcal{F}, T_{s}\right)\left(\tilde{\mathbf{h}}_{l_{s}} \ldots \tilde{\mathbf{h}}_{l_{s}}\right)$ are SI, and there are at least two indices $i$ and $j$ such that $\tilde{\mathbf{h}}_{l_{i}} \neq \tilde{\mathbf{h}}_{l_{j}}$. We call such a monomial a minimal CI monomial.

Proof. The set of CI submonomials of $M(\mathcal{F}, T)$ is finite and it is non-empty since $M(\mathcal{F}, T)$ itself is a CI monomial. We take CI submonomial of $M(\mathcal{F}, T)$ with a minimal rank. Its rank is non-zero since every zero rank submonomial is SI. Since the rank is minimal all submonomials are SI. Hence it has the form (5.30). 


\subsection{Properties of SI monomials}

According to Definition 5.8 for a SI evaluated monomial we have $\tilde{\mathbf{h}}_{l_{1}}=\ldots=\tilde{\mathbf{h}}_{l_{m}}$. Observe also that in view of Definition 2.9 every single-band wavepacket $\tilde{\mathbf{h}}_{l}$ has its band number, and $n^{\prime}=n^{\prime \prime}=\ldots=n^{(m)}$, that is the band $n_{l}=n_{0}$ is the same for all $\tilde{\mathbf{h}}_{l}$. Similarly, $\mathbf{k}_{* l_{1}}=\ldots=\mathbf{k}_{* l_{m}}$. Having these properties we often omit in this section indices $n_{i}, l_{i}$ and skip $\vec{n}$ for notational brevity, writing, for example,

$$
\omega_{n, \zeta}(\mathbf{k})=\omega_{\zeta}(\mathbf{k}), \tilde{\mathbf{u}}_{n, \zeta}(\mathbf{k})=\tilde{\mathbf{u}}_{\zeta}(\mathbf{k}), \chi_{n, \zeta, \vec{n}, \vec{\zeta}}^{(m)}=\chi_{\zeta, \vec{\zeta}}^{(m)} .
$$

\subsubsection{Monomials applied to a single-band wavepacket.}

Here we consider monomials based on oscillatory integral operators and which are applied to a single-band wavepacket. We recall that according to (2.33) a single-band wavepacket $\tilde{\mathbf{h}}$ involves two components $\tilde{\mathbf{h}}_{+}$and $\tilde{\mathbf{h}}_{-}$and a small complement component $\tilde{\mathbf{h}}_{\infty}$.

Definition 5.10 (frequency matching) We call a decorated composition monomial $M(\mathcal{F}, T, \vec{\lambda}, \vec{\zeta})$ frequency matched (FM) if for every non-end node $N \in T$ the corresponding decorated submonomial $M^{\prime}=\mathcal{F}_{\lambda}^{\left(m^{\prime}\right)}\left(M_{1, \zeta^{\prime}} \ldots M_{m^{\prime}, \zeta^{\left(m^{\prime}\right)}}\right)$ satisfies the following conditions:

$$
\lambda \neq \infty, \zeta^{(j)} \neq \infty, j=1, \ldots, m^{\prime}
$$

and

$$
\sum_{j=1}^{m^{\prime}} \zeta^{(j)}=\lambda
$$

where $\lambda, \zeta^{(j)} \in \Lambda$ defined by $\sqrt{4.41}$, we identify \pm with \pm 1 . A decorated composition monomial which does not satisfy the above conditions is called not frequency matched (NFM) monomial.

Collecting separately FM and NFM terms in the expression (4.51) we obtain

$$
\begin{gathered}
M(\mathcal{F}, T)\left(\mathbf{x}_{1} \mathbf{x}_{2} \ldots \mathbf{x}_{m}\right)=\sum_{\mathrm{FM} \vec{\lambda}, \vec{\zeta}} M(\mathcal{F}, T, \vec{\lambda}, \vec{\zeta})\left(\mathbf{x}_{1} \mathbf{x}_{2} \ldots \mathbf{x}_{m}\right) \\
+\sum_{\text {NFM } \vec{\lambda}, \vec{\zeta}} M(\mathcal{F}, T, \vec{\lambda}, \vec{\zeta})\left(\mathbf{x}_{1} \mathbf{x}_{2} \ldots \mathbf{x}_{m}\right) .
\end{gathered}
$$

Remark 5.11 Any SI evaluated monomial is either FM or NFM. We do not define for CI evaluated monomials if they are FM or NFM.

Below we show that FM decorated monomials have the following properties which can be briefly stated as follows. 
Property 1. If $\tilde{\mathbf{h}}(\mathbf{k})$ is a wavepacket in the sense of Definition 2.9 centered around $\pm \mathbf{k}_{*}$ then FM monomial $M(\mathcal{F}, T, \vec{\lambda}, \vec{\zeta})\left(\tilde{\mathbf{h}}^{m}\right)(\mathbf{k})$ is also localized about $\pm \mathbf{k}_{*}$. This property is proved below in Corollary 5.13 .

Property 2. The most important property concerning FM-NFM splitting is that the result of a NFM monomial application to a wavepacket has magnitude $O(\varrho)$, that is $O\left(\beta^{2}\right)$ for the scaling (2.46). Consequently, all NFM terms in (5.33) are small (see Lemma 5.16 below) and they give contribution only to the remainder $\tilde{\mathbf{D}}$ in (2.47).

Now we formulate exact statements clarifying the above properties. The following two statements show, in particular, that an FM monomial transforms a function supported in a vicinity of $\mathbf{k}_{*}$ into a similar function.

Lemma 5.12 (operator support ) If $\tilde{\mathbf{u}}_{1, \zeta^{\prime}} \ldots \tilde{\mathbf{u}}_{m, \zeta^{(m)}}$ are such that

$$
\tilde{\mathbf{u}}_{\zeta^{(l)}}\left(\mathbf{k}^{(l)}\right)=0 \text { when }\left|\mathbf{k}^{(l)}-\zeta^{(l)} \mathbf{k}_{*}\right|>\delta_{l}, l=1, \ldots, m
$$

and

$$
\mathbf{k}_{\vec{\zeta}}=\left(\zeta^{\prime}+\ldots+\zeta^{(m)}\right) \mathbf{k}_{*}
$$

then $\mathcal{F}^{(m)}\left(\tilde{\mathbf{u}}_{1, \zeta^{\prime}} \ldots \tilde{\mathbf{u}}_{m, \zeta^{(m)}}\right)(\mathbf{k}, \tau)$ given by 3.4), satisfies

$$
\mathcal{F}_{\zeta}^{(m)}\left(\tilde{\mathbf{u}}_{1, \zeta^{\prime}} \ldots \tilde{\mathbf{u}}_{m, \zeta^{(m)}}\right)(\mathbf{k}, \tau)=0 \text { if }\left|\mathbf{k}-\mathbf{k}_{\vec{\zeta}}\right|>\delta_{1}+\ldots+\delta_{m} .
$$

In particular, if the binary indices $\zeta, \vec{\zeta}_{(m)}$ are frequency matched $(F M)$, that is

$$
\zeta=\zeta^{\prime}+\ldots+\zeta^{(m)}, \text { where } \zeta^{(j)}, \zeta= \pm 1
$$

then (5.35) holds with $\mathbf{k}_{\vec{\zeta}}=\zeta \mathbf{k}_{*}$.

Proof. ¿From (3.8) and (5.36) we obtain the equality

$$
\mathbf{k}-\zeta \mathbf{k}_{*}=\left(\mathbf{k}^{\prime}-\zeta^{\prime} \mathbf{k}_{*}\right)+\ldots+\left(\mathbf{k}^{(m)}-\zeta^{(m)} \mathbf{k}_{*}\right)
$$

which implies lemma's statement.

Corollary 5.13 (support of a monomial) If $M(\mathcal{F}, T, \vec{\lambda}, \vec{\zeta})\left(\tilde{\mathbf{h}}_{1} \ldots \tilde{\mathbf{h}}_{m}\right)$ is a decorated composition monomial and

$$
\tilde{\mathbf{h}}_{l, \zeta^{(l)}}=0 \text { when }\left|\mathbf{k}^{(l)}-\zeta^{(l)} \mathbf{k}_{*}\right|>\delta_{0}, l=1, \ldots, m
$$

then

$$
M(\mathcal{F}, T, \vec{\lambda}, \vec{\zeta})\left(\tilde{\mathbf{h}}_{1} \ldots \tilde{\mathbf{h}}_{m}\right)(\mathbf{k})=0 \text { if }\left|\mathbf{k}-\mathbf{k}_{\vec{\zeta}}\right|>m \delta_{0}
$$


where $\mathbf{k}_{\vec{\zeta}}$ is defined by 5.34). In particular, if $M(\mathcal{F}, T, \vec{\lambda}, \vec{\zeta})\left(\tilde{\mathbf{h}}_{1} \ldots \tilde{\mathbf{h}}_{m}\right)$ is a FM decorated composition monomial, then

$$
M(\mathcal{F}, T, \vec{\lambda}, \vec{\zeta})\left(\tilde{\mathbf{h}}_{1} \ldots \tilde{\mathbf{h}}_{m}\right)(\mathbf{k})=0 \text { if }\left|\mathbf{k}-\zeta \mathbf{k}_{*}\right|>m \delta_{0}
$$

where $\zeta$ satisfies (5.36). In particular, if $\delta_{0}=\beta^{1-\epsilon}$ and $m \leq C \ln \beta$ then for any $\delta_{1}>0$ there exists $\beta_{0}$ such that for $\beta<\beta_{0}$ we have $C \pi_{0} \beta^{1-\epsilon} \ln \beta<\delta_{1}$ and

$$
M(\mathcal{F}, T, \vec{\lambda}, \vec{\zeta})\left(\tilde{\mathbf{h}}_{1} \ldots \tilde{\mathbf{h}}_{m}\right)(\mathbf{k})=0 \text { when }\left|\mathbf{k}-\zeta \mathbf{k}_{*}\right|>C \pi_{0} \beta^{1-\epsilon} \ln \beta
$$

Proof. To obtain (5.38) we apply Lemma 5.12 and use the induction with respect to the rank of a monomial.

Remark 5.14 If $M(\mathcal{F}, T, \vec{\lambda}, \vec{\zeta})$ is $N F M$ and $\tilde{\mathbf{h}}(\mathbf{k})$ is a wavepacket localized near $\pm \mathbf{k}_{*}$, then $M(\mathcal{F}, T, \vec{\lambda}, \vec{\zeta})\left(\tilde{\mathbf{h}}^{m}\right)(\mathbf{k})$ is localized near the point $\mathbf{k}_{\vec{\zeta}}$. As $\vec{\zeta}$ vary over $\{-1,1\}^{m}$ such points $\mathbf{k}_{\vec{\zeta}}$ lie on a straight line parallel to $\mathbf{k}_{*}$. For $m \rightarrow \infty$ the closure of the set of such $\mathbf{k}_{\vec{\zeta}}$ with a generic $\mathbf{k}_{*}$ can be the entire torus $[-\pi, \pi]^{d}$, whereas for the case of $\vec{\zeta}$ corresponding to an FM monomial the closure is just two points $\pm \mathbf{k}_{*}$. Hence Property 1 is very useful and, in particular, allows to avoid small denominators in coupling terms.

The following lemma shows that the FM interaction phase function of a single wavepacket has a critical point at its center, or, in other words, FM monomials satisfy the group velocity matching condition (see [3], 6]).

Lemma 5.15 If a decorated operator $\mathcal{F}_{\zeta, \vec{\zeta}_{(m)}^{(m)}}$ is FM then the interaction phase function $\phi$ in (3.8) has a critical point:

$$
\nabla_{\mathbf{k}} \phi_{n, \zeta, \vec{n}, \vec{\zeta}}\left(\zeta \mathbf{k}_{*}, \vec{k}_{*}\right)=0 \text { at } \vec{k}_{*}=\left(\zeta^{\prime} \mathbf{k}_{*}, \ldots, \zeta^{(m)} \mathbf{k}_{*}\right) .
$$

Proof. For FM decorated operator all indices $\zeta^{(j)}= \pm$ and

$$
n=n^{\prime}=\ldots=n^{(m)} \text { and } \zeta=\zeta^{\prime}+\ldots+\zeta^{(m)} .
$$

Hence we obtain from (3.9) that

$$
\nabla_{\mathbf{k}} \phi_{n, \zeta, \vec{n}, \vec{\zeta}}(\mathbf{k}, \vec{k})=\zeta \nabla_{\mathbf{k}} \omega(\mathbf{k})-\zeta^{(m)} \nabla_{\mathbf{k}} \omega\left(\mathbf{k}-\mathbf{k}^{\prime}-\ldots-\mathbf{k}^{(m-1)}\right) .
$$

Since $\zeta \mathbf{k}_{*}-\zeta^{\prime} \mathbf{k}_{*}^{\prime}-\ldots-\zeta^{(m-1)} \mathbf{k}_{*}^{(m-1)}=\zeta^{(m)} \mathbf{k}_{*}^{(m)}$ and (2.16) implies

$$
\zeta \nabla_{\mathbf{k}} \omega\left(\zeta \mathbf{k}_{*}\right)=\zeta^{(m)} \nabla_{\mathbf{k}} \omega\left(\zeta^{(m)} \zeta \mathbf{k}_{*}\right) \text { for } \zeta= \pm, \zeta^{(m)}= \pm
$$


we obtain the desired (5.41).

Now we consider NFM monomials and prove the Property 2. First we note that (2.40) implies

$$
\omega_{n_{l}}\left(\mathbf{k}_{* l}\right) \geq \omega_{*}>0, l=1, \ldots, N_{h} .
$$

If $\mathbf{k}_{* l}=\mathbf{k}_{*}, n_{l}=n_{0}$ satisfy Condition 2.13 then if (2.44) does not hold, (2.42) does not hold too, hence for $m \leq m_{F}$

$$
\left|\sum_{j=1}^{m} \zeta^{(j)} \omega_{n_{0}}\left(\mathbf{k}_{*}\right)-\zeta \omega_{n}\left(\mathbf{k}_{\vec{\zeta}}\right)\right| \geq \omega_{*}>0, \mathbf{k}_{\vec{\zeta}}=\sum_{j=1}^{m} \zeta^{(j)} \mathbf{k}_{*},
$$

where $\omega_{*}>0$ is a positive number (we take for notation simplicity the same small enough constsant in (5.44) and (5.45).

The following Lemma, which is a version of the standard statement of the stationary phase method, shows that the action of an NFM monomial on a wavepacket produces a wave of a small amplitude.

Lemma 5.16 Let the decoration projections be defined by (5.1). Assume that Condition 2.13 holds. Let indices $\zeta, \zeta^{\prime}, \ldots, \zeta^{(m)}$ be NFM, that is either one of them is $\infty$ or

$$
\zeta \neq \zeta^{\prime}+\ldots+\zeta^{(m)}, \zeta^{(j)}= \pm 1, \zeta= \pm 1 .
$$

Let $\delta_{N F M}>0$ be small enough to satisfy

$$
\delta_{N F M} \max _{\left|\mathbf{k}_{* l}-\mathbf{k}\right| \leq \delta_{N F M}}\left|\nabla \omega_{l}(\mathbf{k})\right| \leq \frac{1}{4} \omega_{*}, l=1, \ldots, N_{h}
$$

where $\omega_{*}$ is given in 5.45). Let $\mathbf{k}, \mathbf{k}^{(j)}$ satisfy (3.12) and be such that

$$
\sum_{j=1}^{m}\left|\mathbf{k}^{(j)}-\zeta^{(j)} \mathbf{k}_{*}\right| \leq \delta_{N F M},\left|\mathbf{k}-\mathbf{k}_{\vec{\zeta}}\right| \leq \delta_{N F M},
$$

where $\mathbf{k}_{\vec{\zeta}}$ is defined by (5.34) and $\mathbf{k}_{*}=\mathbf{k}_{* l}$ satisfy the conditions (5.44) and 5.45). Let the functions $\tilde{u}_{j, \zeta^{(j)}}(\mathbf{k}, \tau)$ satisfy the condition

$$
\tilde{u}_{j, \zeta^{(j)}}(\mathbf{k}, \tau)=0 \text { when } \zeta^{(j)}=\infty \text { and } \tilde{u}_{j, \zeta^{(j)}}\left(\zeta^{(j)} \mathbf{k}_{*}+\mathbf{s}, \tau\right)=0 \text { when }|\mathbf{s}| \geq \delta_{N F M} .
$$

Then

$$
\begin{gathered}
\left\|\mathcal{F}_{\zeta, \zeta^{\prime}, \ldots, \zeta^{(m)}}^{(m)}\left(\tilde{\mathbf{u}}_{1, \zeta^{\prime}} \ldots \tilde{\mathbf{u}}_{m, \zeta^{(m)}}\right)\right\|_{E} \leq \frac{4 \varrho}{\omega_{*}}\left\|\chi^{(m)}\right\| C_{\Xi}^{2 m+1} \prod_{j}\left\|\tilde{\mathbf{u}}_{j}\right\|_{E} \\
+\frac{2 \varrho \tau_{*}}{\omega_{*}} C_{\Xi}^{2 m+1}\left\|\chi^{(m)}\right\| \sum_{i}\left\|\partial_{\tau} \tilde{\mathbf{u}}_{i}\right\|_{E} \prod_{j \neq i}\left\|\tilde{\mathbf{u}}_{j}\right\|_{E} \cdot
\end{gathered}
$$


Proof. If one of the indices $\zeta^{\prime}, \ldots, \zeta^{(m)}$ equals $\infty$ by (15.49) $\mathcal{F}_{\zeta, \zeta^{\prime}, \ldots, \zeta^{(m)}}^{(m)}=0$ and (15.50) is satisfied. Now we consider the case when all $\zeta, \zeta^{\prime}, \ldots, \zeta^{(m)}$ are finite. We denote for brevity $\omega_{n_{0}}=\omega, \mathbf{k}_{* l}=\mathbf{k}_{*}$ and $\phi_{n, \zeta, \vec{n}, \vec{\zeta}}=\phi$. Since (5.48) holds we get from (3.9) that

$$
\begin{gathered}
\left|\phi(\mathbf{k}, \vec{k})-\phi\left(\mathbf{k}, \vec{k}_{*}\right)\right| \leq\left|\omega\left(\mathbf{k}^{\prime}\right)-\omega\left(\zeta^{\prime} \mathbf{k}_{*}\right)\right|+\ldots+\left|\omega\left(\mathbf{k}^{(m)}\right)-\omega\left(\zeta^{(m)} \mathbf{k}_{*}\right)\right| \\
\quad \leq \max _{\left|\mathbf{k}_{*}-\mathbf{k}\right| \leq \delta_{N F M}}|\nabla \omega(\mathbf{k})| \sum_{j=1}^{m}\left|\mathbf{k}^{(j)}-\zeta^{(j)} \mathbf{k}_{*}\right| \leq \delta_{N F M} \max _{\left|\mathbf{k}_{*}-\mathbf{k}\right| \leq \delta_{N F M}}|\nabla \omega(\mathbf{k})| .
\end{gathered}
$$

Using (5.47) we conclude that

$$
|\phi(\mathbf{k}, \vec{k})| \geq\left|\phi\left(\mathbf{k}, \vec{k}_{*}\right)\right|-\frac{1}{4}\left|\omega_{*}\right| .
$$

By (5.46) the condition (2.44) is not satisfied, therefore (5.45) holds and implies that

$$
\left|\phi\left(\mathbf{k}_{\vec{\zeta}}, \vec{k}_{*}\right)\right| \geq \omega_{*}
$$

Using (15.52), (5.48) and (5.47) we conclude that

$$
\left|\phi\left(\mathbf{k}, \vec{k}_{*}\right)\right| \geq \omega_{*}-\left|\omega(\mathbf{k})-\omega\left(\mathbf{k}_{\vec{\zeta}}\right)\right| \geq \omega_{*}-\delta_{N F M} \max _{\left|\mathbf{k}_{*}-\mathbf{k}\right| \leq \delta_{N F M}}|\nabla \omega(\mathbf{k})| \geq \frac{3}{4} \omega_{*} .
$$

Together with (5.51) this inequality implies that when (5.48) holds we have the estimate

$$
|\phi(\mathbf{k}, \vec{k})| \geq \frac{1}{2} \omega_{*}
$$

Now we note that the oscillatory factor in (3.8)

$$
\exp \left\{\mathrm{i} \phi(\mathbf{k}, \vec{k}) \frac{\tau_{1}}{\varrho}\right\}=\frac{\varrho}{\mathrm{i} \phi(\mathbf{k}, \vec{k})} \partial_{\tau_{1}} \exp \left\{\mathrm{i} \phi(\mathbf{k}, \vec{k}) \frac{\tau_{1}}{\varrho}\right\} .
$$

Integrating (3.8) by parts with respect to $\tau_{1}$ we obtain

$$
\begin{aligned}
& \mathcal{F}_{\zeta, \vec{\zeta}}^{(m)}\left(\tilde{\mathbf{u}}_{1} \ldots \tilde{\mathbf{u}}_{m}\right)(\mathbf{k}, \tau)= \\
& \int_{\mathbb{D}_{m}} \frac{\varrho \exp \left\{\mathrm{i} \phi(\mathbf{k}, \vec{k}) \frac{\tau}{\varrho}\right\}}{\mathrm{i} \phi(\mathbf{k}, \vec{k})} \chi_{\zeta, \vec{\zeta}}^{(m)}(\mathbf{k}, \vec{k}) \tilde{u}_{1, \zeta^{\prime}}\left(\mathbf{k}^{\prime}, \tau\right) \ldots \tilde{u}_{m, \zeta^{\prime}}\left(\mathbf{k}^{(m)}(\mathbf{k}, \vec{k}), \tau\right) \tilde{\mathrm{d}}^{(m-1) d} \vec{k} \\
& -\int_{\mathbb{D}_{m}} \frac{\varrho}{\mathrm{i} \phi(\mathbf{k}, \vec{k})} \chi_{\zeta, \vec{\zeta}}^{(m)}(\mathbf{k}, \vec{k}) \tilde{u}_{1, \zeta^{\prime}}\left(\mathbf{k}^{\prime}, 0\right) \ldots \tilde{u}_{m, \zeta^{\prime}}\left(\mathbf{k}^{(m)}(\mathbf{k}, \vec{k}), 0\right) \tilde{\mathrm{d}}^{(m-1) d} \vec{k} \\
& -\int_{0}^{\tau} \int_{\mathbb{D}_{m}} \frac{\varrho}{\mathrm{i} \phi(\mathbf{k}, \vec{k})} \exp \left\{\mathrm{i} \phi(\mathbf{k}, \vec{k}) \frac{\tau_{1}}{\varrho}\right\}
\end{aligned}
$$

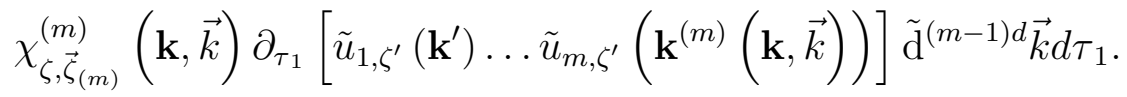


Estimating the denominator by (5.54) and using (3.5) we obtain (5.50). Finally, we consider the case when $\zeta=\infty$ and all remaining indices $\zeta^{(j)}$ equal \pm . We expand $\Pi_{\infty}$ into sum of

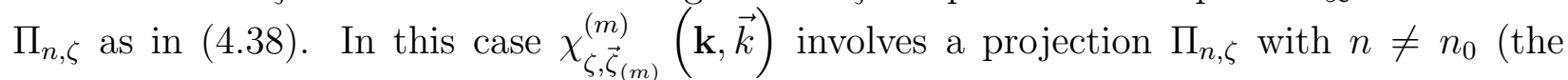
oscillatory integral may involve $N_{h}-1$ terms with such $n$ ). For a fixed $n$ the corresponding phase function $\phi(\mathbf{k}, \vec{k})$ takes the form

$$
\phi(\mathbf{k}, \vec{k})=\phi_{n, \zeta, \vec{n}, \vec{\zeta}}(\mathbf{k}, \vec{k})=\zeta \omega_{n}(\mathbf{k})-\zeta^{\prime} \omega_{n_{0}}\left(\mathbf{k}^{\prime}\right)-\ldots-\zeta^{(m)} \omega_{n_{0}}\left(\mathbf{k}^{(m)}\right) .
$$

Using again (5.45) (now with $n \neq n_{0}$ ) we obtain that (5.52) holds. This implies (5.54) as above provided $\delta_{N F M}$ is small enough. Hence, the relation (5.55) holds, implying readily the desired bound (5.50).

\subsubsection{FM and NFM monomials for SI oscillatory integrals}

The following below theorem shows that NFM monomials are of the order $O(\varrho)$ as $\varrho \rightarrow 0$. We begin first with the following statement.

Lemma 5.17 Assume that Condition 2.13 holds. Let a monomial $S=\mathcal{F}_{\zeta}^{(s)}\left(M_{1, \zeta^{(1)}} \ldots M_{s, \zeta^{(s)}}\right)$ have all submonomials $M_{1, \zeta^{(1)}} \ldots M_{s, \zeta^{(s)}}$ which satisfy $F M$ condition (5.36), but $S$ itself is not FM. Assume that $S$ is applied to wavepackets $\mathbf{h}_{l}$ which satisfy Definition [2.9. and

$$
\tilde{\mathbf{h}}_{l, \zeta}(\mathbf{k}, \beta)=0 \text { if }\left|\mathbf{k}-\zeta \mathbf{k}_{* l}\right| \geq \pi_{0} \beta^{1-\epsilon}, \zeta= \pm .
$$

Then

$$
\begin{gathered}
\|S\|_{E} \leq \frac{4 \varrho\left\|\chi^{(s)}\right\|}{\left|\omega\left(\mathbf{k}_{*}\right)\right|} C_{\Xi}^{2 s+1} \prod_{j}\left\|M_{j, \zeta^{(j)}}\right\|_{E}+ \\
+\frac{4 \varrho \tau_{*}\left\|\chi^{(s)}\right\|}{\left|\omega\left(\mathbf{k}_{*}\right)\right|} C_{\Xi}^{2 s+1} \sum_{i=1}^{s}\left\|\partial_{\tau} M_{i, \zeta^{(i)}}\right\|_{E} \prod_{j \neq i}\left\|M_{j, \zeta^{(j)}}\right\|_{E}, E=C\left(\left[0, \tau_{*}\right], L_{1}\right) .
\end{gathered}
$$

Proof. Since $M_{1, \zeta^{(1)}} \ldots M_{s, \zeta^{(s)}}$ are decorated FM submonomials we can use Lemma 5.12 and Corollary 5.13. Applying Corollary 5.13 and using (5.12) we obtain that

$$
M_{l, \zeta^{(l)}}\left(\mathbf{k}^{(l)}, \tau_{1}\right)=0 \text { when }\left|\mathbf{k}^{(l)}-\zeta^{(l)} \mathbf{k}_{*}\right|>\nu\left(M_{l, \zeta^{(l)}}\right) \beta^{1-\epsilon} \pi_{0}, l=1, \ldots, s .
$$

where $\nu(M)$ is homogeneity index of $M$. Consider now the oscillatory integral (3.8) which determines $S$, namely

$$
\begin{gathered}
\mathcal{F}_{\zeta, \vec{\zeta}}^{(s)}\left(M_{1, \zeta^{(1)}} \ldots M_{s, \zeta^{(s)}}\right)(\mathbf{k}, \tau)=\int_{0}^{\tau} \int_{\mathbb{D}_{s}} \exp \left\{\mathrm{i} \phi_{\zeta, \vec{\zeta}}(\mathbf{k}, \vec{k}) \frac{\tau_{1}}{\varrho}\right\} \\
\chi_{\zeta, \vec{\zeta}}^{(s)}(\mathbf{k}, \vec{k}) M_{1, \zeta^{(1)}}\left(\mathbf{k}^{\prime}, \tau_{1}\right) \ldots M_{s, \zeta^{(s)}}\left(\mathbf{k}^{(s)}(\mathbf{k}, \vec{k}), \tau_{1}\right) \tilde{\mathrm{d}}^{(s-1) d} \vec{k} \mathrm{~d} \tau_{1} .
\end{gathered}
$$


We apply Lemma [5.16] where, according to (5.58) and (5.62) $\delta_{N F M}=m \beta^{1-\epsilon} \pi_{0}$. According to (5.50)

$$
\begin{aligned}
\|S\|_{E} & =\left\|\mathcal{F}_{\zeta, \vec{\zeta}_{(s)}^{(s)}}\left(M_{1, \zeta^{(1)}} \ldots M_{s, \zeta^{(s)}}\right)(\mathbf{k}, \tau)\right\|_{E} \leq \frac{4 \varrho\left\|\chi^{(s)}\right\|}{\left|\omega\left(\mathbf{k}_{*}\right)\right|} C_{\Xi}^{2 s+1} \prod_{j}\left\|M_{j, \zeta^{(j)}}\right\|_{E} \\
& +\frac{4 \varrho \tau_{*}\left\|\chi^{(s)}\right\|}{\left|\omega\left(\mathbf{k}_{*}\right)\right|} C_{\Xi}^{2 s+1} \sum_{i=1}^{s}\left\|\partial_{\tau} M_{i, \zeta^{(i)}}\right\|_{E} \prod_{j \neq i}\left\|M_{j, \zeta^{(j)}}\right\|_{E}, E=C\left(\left[0, \tau_{*}\right], L_{1}\right),
\end{aligned}
$$

that implies (5.57).

Theorem 5.18 Suppose that (i) the inequalities 5.44) hold; (ii) $\tilde{\mathbf{h}}_{l}$ are wavepackets in the sense of Definition [2.9; (iii) the relations (5.56) hold; (iv) the projections are defined by (5.1); (v) Condition 2.13 holds. Then a NFM decorated monomial based on oscillatory integrals $\mathcal{F}$ defined by 3.4) satisfies the estimate

$$
\begin{gathered}
\left\|M(\mathcal{F}, T, \vec{\lambda}, \vec{\zeta})\left(\tilde{\mathbf{h}}_{1} \ldots \tilde{\mathbf{h}}_{m}\right)\right\|_{C\left(\left[0, \tau_{*}\right], L_{1}\right)} \leq \\
\frac{4 \varrho \tau_{*}^{i-1}[1+m]}{\left|\omega\left(\mathbf{k}_{*}\right)\right|} C_{\Xi}^{2 i+e} \prod_{N \in T, r(N)>0}\left\|\chi^{(\mu(N))}\right\| \prod_{l=1}^{m}\left\|\tilde{\mathbf{h}}_{l, \zeta^{(l)}}\right\|_{C\left(\left[0, \tau_{*}\right], L_{1}\right)},
\end{gathered}
$$

where $i, m$ and e are respectively the incidence number, the homogeneity index and the number of edges of $T$.

Proof. Let $M\left(\mathcal{F}, T, \vec{\lambda}_{(q)}, \vec{\zeta}_{(m)}\right)\left(\tilde{\mathbf{h}}_{1} \ldots \tilde{\mathbf{h}}_{m}\right)$ be a NFM decorated $m$-homogenious monomial. We find a decorated submonomial $S=M\left(\mathcal{F}, T\left(N_{0}\right), \vec{\lambda}_{(q)}, \vec{\zeta}_{(m)}\right)$ of $M\left(\mathcal{F}, T, \vec{\lambda}_{(q)}, \vec{\zeta}_{(m)}\right)$ with such $N_{0}$ that $S$ is NFM and has minimal rank of all NFM submonomials. We denote by $r_{0}$ the rank of $S$, by $i^{\prime}$ its incidence number and by $s=\nu(S)=\nu\left(T\left(N_{0}\right)\right)$ its homogeneity index. This monomial has the form $S=\mathcal{F}_{\zeta}^{(s)}\left(M_{1, \zeta^{(1)}} \ldots M_{s, \zeta^{(s)}}\right)$. Since the rank is minimal, all decorated submonomials $M_{l, \zeta^{(l)}}$ are $F M$ and their ranks do not exceed $r_{0}-1$. Then according to (4.21) their homogeneity indices satisfy

$$
\nu\left(M_{1, \zeta^{(1)}}\right)+\ldots+\nu\left(M_{s, \zeta^{(s)}}\right)=s \leq m
$$

Applying Lemma 5.17 we obtain (5.57). Now we use Lemma 5.1 and 5.2, Applying Lemma 4.24 we obtain

$$
\begin{gathered}
\left\|M(\{\mathcal{F}\}, T, \Gamma)\left(\tilde{\mathbf{h}}_{1} \ldots \tilde{\mathbf{h}}_{m}\right)\right\|_{E} \leq \\
\|S\|_{E} \prod_{N \in T \backslash T^{\prime}\left(N_{0}\right), r(N)>0}\left\|\mathcal{F}_{\Gamma(N)}^{(\mu(N))}\right\| \prod_{l<\varkappa}\left\|\tilde{\mathbf{h}}_{l, \zeta^{(l)}}\right\|_{E} \prod_{l \geq \varkappa+\nu\left(T^{\prime}\left(N_{0}\right)\right)}\left\|\tilde{\mathbf{h}}_{l, \zeta^{(l)}}\right\|_{E} .
\end{gathered}
$$


Note that the norm of $\left\|\mathcal{F}_{\Gamma(N)}^{(\mu(N))}\right\|$ is estimated by (15.2) and norm of $S$ by (15.57). In turn, we estimate right-hand side of (5.57) using (5.2) and (5.3) Taking into account that $s \leq m$ in the sum in (5.60) we get the estimate (5.61).

We also consider the case when Condition 2.13 does not hold and Condition 2.23 holds. In this case we give an alternative definition of FM and NFM decorated monomials.

Definition 5.19 (Alternative Frequency Matching) We call a decorated composition monomial $M(\mathcal{F}, T, \vec{\lambda}, \vec{\zeta})$ alternatively frequency matched (AFM) if (i) every node of $T$ has an odd number of child nodes (at least three); (ii) for every non-end node $N \in T$ the corresponding decorated submonomial $M^{\prime}(\mathcal{F}, T(N), \vec{\lambda}, \vec{\zeta})=\mathcal{F}_{\lambda}^{\left(m^{\prime}\right)}\left(M_{1, \zeta^{\prime}} \ldots M_{m^{\prime}, \zeta^{\left(m^{\prime}\right)}}\right)$ satisfies (5.31) and

$$
\operatorname{sign}\left(\sum_{j=1}^{m^{\prime}} \zeta^{(j)}\right)=\lambda,
$$

where $\lambda, \zeta^{(j)} \in \Lambda$ defined by (4.41), we identify \pm with \pm 1 . A decorated composition monomial which is not $A F M$ is called alternatively not frequency matched (ANFM) monomial.

Now we prove a statement analogous to Theorem 5.18 when Condition 2.23 holds.

Theorem 5.20 Assume that assumptions of Theorem NFM hold with Condition 2.13 replaced by Condition 2.23. Then (5.61) holds.

Proof. According to Corollary [5.13, if $\tilde{\mathbf{h}}_{l_{1}}=\ldots=\tilde{\mathbf{h}}_{l_{m}}=\tilde{\mathbf{h}}_{l}$ satisfy Definition [2.9] and (15.56), then $M(\mathcal{F}, T, \vec{\lambda}, \vec{\zeta})\left(\tilde{\mathbf{h}}_{l_{1}} \ldots \tilde{\mathbf{h}}_{l_{m}}\right)=M(\mathcal{F}, T, \vec{\lambda}, \vec{\zeta})\left(\tilde{\mathbf{h}}_{l_{1}, \zeta^{\prime}} \ldots \tilde{\mathbf{h}}_{l_{m}, \zeta^{(m)}}\right)$ has support in a $m \beta^{1-\epsilon}$ vicinity of $\mathbf{k}_{\vec{\zeta}}=\nu \mathbf{k}_{*}$ defined by (5.34), $\nu$ and $m$ are odd integers, $m \geq 3, \nu=$ $\zeta^{\prime}+\ldots+\zeta^{(m)}$. Let $S=M\left(\mathcal{F}, T^{\prime}, \vec{\lambda}, \vec{\zeta}\right)$ be minimal ANFM submonomial of $M(\mathcal{F}, T, \vec{\lambda}, \vec{\zeta})$, that is if $T^{\prime \prime} \subset T^{\prime}$ then $M\left(\mathcal{F}, T^{\prime \prime}, \vec{\lambda}, \vec{\zeta}\right)$ is AFM submonomial of $S$. The monomial $S$ has the form of (5.59) with the interaction phase function

$$
\phi_{\zeta, \vec{\zeta}}(\mathbf{k}, \vec{k})=\zeta \omega_{n}(\mathbf{k})-\zeta^{\prime} \omega_{n_{0}}\left(\mathbf{k}^{\prime}\right)-\ldots-\zeta^{(s)} \omega_{n_{0}}\left(\mathbf{k}^{(s)}\right)
$$

The integrand is non-zero near $\mathbf{k}^{(j)}=\nu_{j} \mathbf{k}_{*}$, and applying (5.63) to every AFM submonomial we get

$$
\zeta^{(l)}=\operatorname{sign}\left(\nu_{l}\right)
$$

Using (2.96) and (2.16) we obtain

$$
\begin{aligned}
& \phi_{\zeta, \vec{\zeta}}\left(\nu \mathbf{k}_{*}, \vec{k}_{*}\right)=\zeta \omega_{n}\left(\nu \mathbf{k}_{*}\right)-\zeta^{\prime} \omega_{n_{0}}\left(\nu_{1} \mathbf{k}_{*}\right)-\ldots-\zeta^{(s)} \omega_{n_{0}}\left(\nu_{s} \mathbf{k}_{*}\right) \\
& =\zeta \omega_{n}\left(\nu \mathbf{k}_{*}\right)-\operatorname{sign}\left(\nu_{1}\right)\left|\nu_{1}\right| \omega_{n_{0}}\left(\mathbf{k}_{*}\right)-\ldots-\operatorname{sign}\left(\nu_{s}\right)\left|\nu_{s}\right| \omega_{n_{0}}\left(\mathbf{k}_{*}\right) \\
& =\zeta|\nu| \omega_{n}\left(\mathbf{k}_{*}\right)-\left(\nu_{1}+\ldots+\nu_{s}\right) \omega_{n_{0}}\left(\mathbf{k}_{*}\right), \nu=\nu_{1}+\ldots+\nu_{s} .
\end{aligned}
$$


Therefore, since $S$ is $A N F M, \zeta \neq \operatorname{sign}(\nu)$ and since $\nu$ is odd,

$$
\phi_{\zeta, \vec{\zeta}}\left(\nu \mathbf{k}_{*}, \vec{k}_{*}\right)=-2 \nu \omega_{n_{0}}\left(\mathbf{k}_{*}\right) \neq 0
$$

therefore (5.52) holds. We can repeat the proofs of Lemma 5.16 and Lemma 5.17 and obtain (5.57). From (5.57) we obtain (5.61) as in the proof of Theorem 5.18.

Below we give estimations for the derivatives with respect to $\mathbf{k}$ of a composition monomial applied to a wavepacket. Note that (2.35) admits a singular dependence on $\beta$ of wavepackets $\tilde{h}_{\zeta}(\beta, \mathbf{k})$. This type of dependence also naturally comes from explicit formulas as (2.36) which yield that the first derivative with respect to $\mathbf{k}$ has a factor $\beta^{-1}$. Below we estimate dependence on $\beta$ of monomials applied to wavepackets and will show that they have the same type of singularity.

Observe that by (5.13) all the points $\mathbf{k}_{* l}$ are at the distance at least $2 \pi_{0}$ from $\sigma$. Hence, according to Definition 2.3, and (2.28)

$$
\begin{gathered}
\max _{\left|\mathbf{k} \pm \mathbf{k}_{* l}\right| \leq \pi_{0}, l=1, \ldots, N_{h},}\left(\left|\nabla_{\mathbf{k}}^{2} \omega\right|+\left|\nabla_{\mathbf{k}} \omega\right|\right) \leq C_{\omega, 2}, \\
\max _{\left|\mathbf{k} \pm \mathbf{k}_{* l}\right| \leq \pi_{0}, l=1, \ldots, N_{h}}\left|\nabla \chi_{\zeta, \vec{\zeta}}^{(m)}\left(\mathbf{k}, \mathbf{k}^{\prime}, \ldots, \mathbf{k}^{(m)}\right)\right| \leq C_{\chi} C_{\Xi}^{m+1} .
\end{gathered}
$$

The following seemingly technical Lemma describes a very important property of solutions. It shows that the k-gradient of solutions behaves, roughly speaking, as the gradient of initial data. Corresponding estimates play a crucial role in the control of smallness of interaction of different wavepackets.

Lemma 5.21 Let $M(\mathcal{F}, T, \vec{\lambda}, \vec{\zeta})\left(\tilde{\mathbf{h}}_{l_{1}} \ldots \tilde{\mathbf{h}}_{l_{m}}\right)$ be a decorated monomial which is SI. Assume that $\tilde{\mathbf{h}}_{l_{j}}=\tilde{\mathbf{h}}_{l}$ are wavepackets satisfying Definition 2.9, (5.56) and (5.19), that 2.46) holds and

$$
\beta^{1-\epsilon} m \leq \pi_{0} .
$$

Assume that either Condition 2.13 holds and the monomial is FM or Condition 2.23 holds and the monomial is AFM. Then

$$
\left\|\nabla_{\mathbf{k}} M(\mathcal{F}, T, \vec{\lambda}, \vec{\zeta})\left(\tilde{\mathbf{h}}_{l_{1}} \ldots \tilde{\mathbf{h}}_{l_{m}}\right)\right\|_{E} \leq C C_{\chi} \tau_{*}^{i} C_{\Xi}^{2 i+e} C_{\chi}^{i-1} R^{m-1} \beta^{-1-\epsilon} m^{2}
$$

where $E=C\left(\left[0, \tau_{*}\right], L_{1}\right), \tau_{*} \leq 1$, with $i=i(T)$ and $e=e(T)$ being respectively the incidence number and the number of edges of $T$.

Proof. We use the induction with respect to the incidence number $i$ of a tree $T$. First, we consider the case when Condition 2.13 holds and $M(\mathcal{F}, T, \vec{\lambda}, \vec{\zeta})$ is FM. For $i=0$ (5.71) follows from (2.35). Now we assume that (5.71) holds for the incidence number less than $i$ 
and prove it when the incidence number equals $i$. Since arguments of $M(\mathcal{F}, T, \vec{\lambda}, \vec{\zeta})$ are SI, according Definition $5.8 l_{1}=\ldots=l_{m}=l$. It is sufficient to prove the boundedness of

$$
\left\|\nabla_{\mathbf{k}} M(\mathcal{F}, T, \vec{\lambda}, \vec{\zeta})\left(\tilde{\mathbf{h}}_{l}^{m}\right)\right\|_{E}=\left\|\nabla_{\mathbf{k}} \mathcal{F}_{\lambda}^{(s)}\left(M_{1, \zeta^{\prime}} \ldots M_{s, \zeta^{(s)}}\right)\right\|_{E},
$$

where $M_{1} \ldots M_{s}$ are decorated submonomials, $M_{j, \zeta}=\Pi_{\zeta} M_{j}$. Let the submonomials have incidence numbers $i_{1}, \ldots, i_{s}$ and homogeneities $m_{1}, \ldots, m_{s}$ respectively satisfying

$$
i_{1}+\ldots+i_{s}=i-1, m_{1}+\ldots+m_{s}=m .
$$

We have by (3.8)

$$
\begin{gathered}
\nabla_{\mathbf{k}} \mathcal{F}_{\lambda}^{(s)}\left(M_{1, \zeta^{\prime}} \ldots M_{s, \zeta^{(s)}}\right)(\mathbf{k}, \tau)=\nabla_{\mathbf{k}} \int_{0}^{\tau} \int_{[-\pi, \pi]^{(s-1) d}} \exp \left\{\operatorname{i\phi }{ }_{\lambda, \vec{\zeta}}(\mathbf{k}, \vec{k}) \frac{\tau_{1}}{\varrho}\right\} \\
\chi_{\lambda, \vec{\zeta}}^{(s)}(\mathbf{k}, \vec{k}) M_{1, \zeta^{\prime}}\left(\mathbf{k}^{\prime}\right) \ldots M_{s, \zeta^{(s)}}\left(\mathbf{k}^{(s)}(\mathbf{k}, \vec{k})\right) \tilde{\mathrm{d}}^{(s-1) d} \vec{k} d \tau_{1} .
\end{gathered}
$$

By Leibnitz formula

$$
\nabla_{\mathbf{k}} \mathcal{F}_{\lambda}^{(s)}\left(M_{1, \zeta^{\prime}} \ldots M_{s, \zeta^{(s)}}\right)(\mathbf{k}, \tau)=I_{1}+I_{2}+I_{3},
$$

where

$$
\begin{gathered}
I_{1}=\int_{0}^{\tau} \int_{[-\pi, \pi]^{(s-1) d}}\left[\nabla_{\mathbf{k}} \exp \left\{\mathrm{i} \phi_{\lambda, \vec{\zeta}}(\mathbf{k}, \vec{k}) \frac{\tau_{1}}{\varrho}\right\}\right] \\
\chi_{\lambda, \vec{\zeta}}^{(s)}(\mathbf{k}, \vec{k}) M_{1, \zeta^{\prime}}\left(\mathbf{k}^{\prime}\right) \ldots M_{s, \zeta^{(s)}}\left(\mathbf{k}^{(s)}(\mathbf{k}, \vec{k})\right) \tilde{\mathrm{d}}^{(s-1) d} \vec{k} d \tau_{1}, \\
I_{2}=\int_{0}^{\tau} \int_{[-\pi, \pi]^{(s-1) d}} \exp \left\{\mathrm{i} \phi_{\lambda, \vec{\zeta}}(\mathbf{k}, \vec{k}) \frac{\tau_{1}}{\varrho}\right\} \\
{\left[\nabla_{\mathbf{k}} \chi_{\lambda, \vec{\zeta}}^{(s)}(\mathbf{k}, \vec{k})\right] M_{1, \zeta^{\prime}}\left(\mathbf{k}^{\prime}\right) \ldots M_{s, \zeta^{(s)}}\left(\mathbf{k}^{(s)}(\mathbf{k}, \vec{k})\right) \tilde{\mathrm{d}}^{(s-1) d} \vec{k} d \tau_{1},} \\
I_{3}=\int_{0}^{\tau} \int_{[-\pi, \pi]^{(s-1) d}} \exp \left\{\mathrm{i} \phi{ }_{\lambda, \vec{\zeta}}(\mathbf{k}, \vec{k}) \frac{\tau_{1}}{\varrho}\right\} \\
\chi_{\lambda, \vec{\zeta}}^{(s)}(\mathbf{k}, \vec{k}) M_{1, \zeta^{\prime}}\left(\mathbf{k}^{\prime}\right) \ldots \nabla_{\mathbf{k}} M_{s, \zeta^{(s)}}\left(\mathbf{k}^{(s)}(\mathbf{k}, \vec{k})\right) \tilde{\mathrm{d}}^{(s-1) d} \vec{k} d \tau_{1} .
\end{gathered}
$$

By (5.5)

$$
\left\|M_{j, \zeta^{(j)}}\left(\mathbf{k}^{(j)}\right)\right\|_{L_{1}} \leq C \tau^{i^{(j)}} C_{\Xi}^{2 i^{(j)}+e^{(j)}} C_{\chi}^{i^{(j)}} R^{m_{j}}, j=1, \ldots, s .
$$

Using (3.5), (5.75), (5.72) and the induction assumption we get

$$
\left|I_{3}\right| \leq\left\|\chi^{(s)}\right\| \prod_{j=1}^{s-1}\left\|M_{j, \zeta^{(j)}}\left(\mathbf{k}^{(j)}\right)\right\|_{E} \int_{0}^{\tau}\left\|\nabla_{\mathbf{k}} M_{s, \zeta^{(s)}}\right\|_{E} d \tau_{1} \leq C C_{1}^{m} R^{m-1} \tau^{i} C_{\Xi}^{2 i+e} C_{\chi}^{i} \beta^{-1-\epsilon} .
$$


From (15.75) and the smoothness of $\chi^{(s)}(\mathbf{k}, \vec{k})$ we get

$$
\left|I_{2}\right| \leq C \beta^{-1-\epsilon} \tau^{i} C_{1}^{m} C_{\Xi}^{2 i+e} C_{\chi}^{i} R^{m}
$$

Now we estimate $I_{1}$. Using (3.9) we obtain

$$
\begin{gathered}
I_{1}=\int_{0}^{\tau} \int_{[-\pi, \pi]^{(s-1) d}}\left[\exp \left\{\mathrm{i} \phi_{\lambda, \vec{\zeta}}(\mathbf{k}, \vec{k}) \frac{\tau_{1}}{\varrho}\right\}\right] \\
\frac{\tau_{1}}{\varrho}\left[-\lambda \nabla_{\mathbf{k}} \omega(\mathbf{k})+\zeta^{(s)} \nabla_{\mathbf{k}} \omega\left(\mathbf{k}^{(s)}(\mathbf{k}, \vec{k})\right)\right] \\
\chi_{\lambda, \vec{\zeta}}^{(s)}(\mathbf{k}, \vec{k}) M_{1, \zeta^{\prime}}\left(\mathbf{k}^{\prime}\right) \ldots M_{s, \zeta^{(s)}}\left(\mathbf{k}^{(s)}(\mathbf{k}, \vec{k})\right) \tilde{\mathrm{d}}^{(s-1) d} \vec{k} d \tau_{1} .
\end{gathered}
$$

The difficulty in the estimation of the integral $I_{1}$ comes from the factor $\frac{\tau_{1}}{\varrho}$ since $\varrho$ is small. Note that according to (2.46) $\beta^{2} / \varrho \leq C$. Since $M(\mathcal{F}, T, \vec{\lambda}, \vec{\zeta})$ is FM, its every submonomial is FM too and we can apply to them Corollary 5.13, which yields

$$
M_{j, \zeta^{(j)}}\left(\mathbf{k}^{(j)}\right)=0 \text { for }\left|\mathbf{k}^{(j)}-\zeta^{(j)} \mathbf{k}_{*}\right|>m_{j} \pi_{0} \beta^{1-\epsilon}, j=1, \ldots, s .
$$

Hence, it is sufficient to estimate $I_{1}$ for.

$$
\left|\mathbf{k}^{(j)}-\zeta^{(j)} \mathbf{k}_{*}\right| \leq \delta_{1}=m \pi_{0} \beta^{1-\epsilon} \text { for all } j
$$

According to Lemma 5.15 , since $\vec{\lambda}, \vec{\zeta}$ are FM

$$
\nabla_{\mathbf{k}} \phi_{\lambda, \vec{\zeta}}\left(\lambda \mathbf{k}_{*}, \vec{k}_{*}\right)=\left[-\lambda \nabla_{\mathbf{k}} \omega\left(\mathbf{k}_{*}\right)+\zeta^{(s)} \nabla_{\mathbf{k}} \omega\left(\left(\mathbf{k}^{(s)}\left(\mathbf{k}_{*}, \vec{k}_{*}\right)\right)\right)\right]=0 .
$$

Using (15.68) we conclude that in a vicinity of $\mathbf{k}_{*}$ defined by (15.79) we have

$$
\left|\left[-\lambda \nabla_{\mathbf{k}} \omega(\mathbf{k})+\zeta^{(s)} \nabla_{\mathbf{k}} \omega\left(\mathbf{k}^{(s)}(\mathbf{k}, \vec{k})\right)\right]\right| \leq 2(s+1) C_{\omega, 2} \delta_{1} .
$$

This yields the estimate

$$
\left|I_{1}\right| \leq C C_{\Xi}^{2 i+e} \tau^{i} C_{\chi}^{i} C_{1}^{m} \beta^{-1-\epsilon} m^{2} R^{m} .
$$

Combining (5.81), (5.77) and (5.76) we obtain (5.71) and the induction is completed. Now we consider the case when Condition 2.23 holds and the monomial is AFM. Note that according to Corollary 5.13 the submonomials $M_{j, \zeta^{(j)}}$ have supports near $\nu_{j} \mathbf{k}_{*}$, with an odd $\nu_{j}$. By Lemma 5.12 the monomial itself is non-zero near $\nu \mathbf{k}_{*}, \nu=\nu_{1}+\ldots+\nu_{s}$; since $s$ is odd $\nu$ is odd too. Obviously, one of $\nu_{j}$ has the same sign as $\nu$, we assume that $j=s$, that is

$$
\operatorname{sign}\left(\nu_{s}\right)=\operatorname{sign}\left(\nu_{1}+\ldots+\nu_{s}\right)=\operatorname{sign}(\nu),
$$


the general case can be reduced to this by a relabeling of variables. The interaction phase function is given by (5.64) and since the submonomials are AFM (5.65) holds. According to (2.16) $\nabla_{\mathbf{k}}(\omega(-\mathbf{k}))=-\left(\nabla_{\mathbf{k}} \omega\right)(\mathbf{k})$. Therefore, using (2.95) we obtain

$$
\begin{gathered}
\nabla_{\mathbf{k}} \phi_{\lambda, \vec{\zeta}}\left(\nu \mathbf{k}_{*}, \vec{k}_{*}\right)=\lambda \nabla_{\mathbf{k}} \omega\left(\nu \mathbf{k}_{*}\right)-\zeta^{(s)} \nabla_{\mathbf{k}} \omega\left(\nu_{s} \mathbf{k}_{*}\right)= \\
\lambda\left(\nabla_{\mathbf{k}} \omega\right)\left(\operatorname{sign}(\nu)|\nu| \mathbf{k}_{*}\right)-\zeta^{(s)} \nabla_{\mathbf{k}} \omega\left(\operatorname{sign}\left(\nu_{s}\right)\left|\nu_{s}\right| \mathbf{k}_{*}\right) \\
=\lambda\left(\nabla_{\mathbf{k}} \omega\right)\left(\operatorname{sign}(\nu) \mathbf{k}_{*}\right)-\zeta^{(s)} \nabla_{\mathbf{k}} \omega\left(\operatorname{sign}\left(\nu_{s}\right) \mathbf{k}_{*}\right)=\left(\lambda \operatorname{sign}(\nu)-\zeta^{(s)} \operatorname{sign}\left(\nu_{s}\right)\right)\left(\nabla_{\mathbf{k}} \omega\right)\left(\mathbf{k}_{*}\right) .
\end{gathered}
$$

Using (15.65) we conclude that

$$
\nabla_{\mathbf{k}} \phi_{\lambda, \vec{\zeta}}\left(\nu \mathbf{k}_{*}, \vec{k}_{*}\right)=0, \vec{k}_{*}=\left(\nu_{1} \mathbf{k}_{*}, \ldots, \nu_{s} \mathbf{k}_{*}\right)
$$

Using (5.83) instead of (5.80) we conclude as in the first half of the proof that (5.71) holds in the AFM case too.

\subsection{Properties of minimal CI monomials}

Here we consider CI evaluated monomials with arguments involving different wavepackets $\tilde{\mathbf{h}}_{l}$. Since the group velocities of wavepackets are different, namely (2.41) is satisfied, there exists $p_{0}>0$ such that

$$
\left|\nabla \omega\left(\mathbf{k}_{* l_{1}}\right)-\nabla \omega\left(\mathbf{k}_{* l_{2}}\right)\right| \geq p_{0}>0 \text { if } l_{1} \neq l_{2} .
$$

The next lemma is a standard implication of the Stationary Phase Method in the case when the phase function has no critical points in the domain of integration, namely when (2.41) holds.

Lemma 5.22 Let $\mathbf{k}_{* l}$ and $\omega_{n}$ be generic in the sense of Definition 2.24. Let $\mathcal{F}^{(m)}$ be defined by (3.4), $m(\beta)$ be as in (5.23). We assume that (2.28) and (2.41) hold. We also assume that (5.19), (5.56), 2.34), 2.35) and 2.46) hold. We assume that $M(\mathcal{F}, T)\left(\tilde{\mathbf{h}}_{l_{1}} \ldots \tilde{\mathbf{h}}_{l_{m}}\right)$ is a monomial with homogeneity index $m$ evaluated at arguments with $C I$ multiindex $l_{1}, \ldots, l_{m}$, but every evaluated submonomial of $M(\mathcal{F}, T)\left(\tilde{\mathbf{h}}_{l_{1}} \ldots \tilde{\mathbf{h}}_{l_{m}}\right)$ is SI. Then for $m \leq m(\beta)$ and small $\beta$

$$
\left\|M(\mathcal{F}, T)\left(\tilde{\mathbf{h}}_{l_{1}} \ldots \tilde{\mathbf{h}}_{l_{m}}\right)\right\|_{E} \leq \frac{C}{p_{0}} \tau_{*}^{i-1} C_{\Xi}^{2 i+e} 3^{2 m} C_{\chi}^{i}\left[\frac{\varrho|\ln \beta|}{\beta^{1+\epsilon}}+\beta\right] m^{2} R^{m-1},
$$

where $i$ and e are respectively the incidence number and number of edges of $T, R$ is as in (5.19).

Proof. Since $\mathbf{k}_{* l}$ are not band-crossing points, the relations (5.69) and (5.68) hold. We expand $M(\mathcal{F}, T)$ into a sum of decorated monomials $M(\mathcal{F}, T, \vec{\lambda}, \vec{\zeta})$ as in (4.51), which 
contains no more than $3^{i(T)+m}$ terms, and $i(T)+m \leq 2 m$. The submonomials of every decorated monomial are SI by the assumption of the theorem. If Condition 2.13 holds, the submonomials are either FM or NFM; if Condition 2.23 holds, the submonomials are either AFM or ANFM. If a decorated submonomial $M\left(\mathcal{F}, T^{\prime}, \vec{\lambda}^{\prime}, \vec{\zeta}^{\prime}\right)$ is NFM we use Theorem 5.18 and obtain from (5.61) the inequality

$$
\left\|M\left(\mathcal{F}, T^{\prime}, \vec{\lambda}^{\prime}, \vec{\zeta}^{\prime}\right)\left(\tilde{\mathbf{h}}_{l_{j^{\prime}+1}} \ldots \tilde{\mathbf{h}}_{l_{j^{\prime}+m^{\prime}}}\right)\right\|_{E} \leq C \varrho \tau_{*}^{i^{\prime}-1}[1+m] C_{\Xi}^{2 i^{\prime}+e^{\prime}} C_{\chi}^{i^{\prime}} R^{m^{\prime}},
$$

where $i^{\prime}$ and $e^{\prime}$ are the incidence number and number of edges of the subtree $T^{\prime}$. Alternatively, if Condition 2.23 holds, and a decorated monomial $M\left(\mathcal{F}, T^{\prime}, \vec{\lambda}^{\prime}, \vec{\zeta}^{\prime}\right)$ is ANFM, we use Theorem 5.20 and obtain from (5.61) the inequality (5.86). Using (5.86) in both cases we obtain

$$
\left\|M(\mathcal{F}, T, \vec{\lambda}, \vec{\zeta})\left(\tilde{\mathbf{h}}_{l_{1}} \ldots \tilde{\mathbf{h}}_{l_{m}}\right)\right\|_{E} \leq C \varrho \tau_{*}^{i-1} C_{\Xi}^{2 i+e} C_{\chi}^{i} m R^{m}
$$

Now we consider the case when Condition 2.13 holds and every submonomial of $M(\mathcal{F}, T, \vec{\lambda}, \vec{\zeta})$ is FM. We write the integral with respect to $\tau_{1}$ in (5.59) as a sum of two integrals from 0 to $\beta$ and from $\beta$ to $\tau$, namely

$$
\begin{gathered}
\mathcal{F}_{\zeta, \vec{\zeta}}^{(s)}\left(M_{1} \ldots M_{s}\right)(\mathbf{k}, \tau)=F_{1}+F_{2}, \\
F_{1}=\int_{\beta}^{\tau} \int_{\mathbb{D}_{m}} \exp \left\{\mathrm{i} \phi_{\zeta, \vec{\zeta}}(\mathbf{k}, \vec{k}) \frac{\tau_{1}}{\varrho}\right\} A_{\zeta, \vec{\zeta}}^{(s)}(\mathbf{k}, \vec{k}) \tilde{\mathrm{d}}^{(s-1) d} \vec{k} d \tau_{1}, F_{2}=\int_{0}^{\beta} \ldots d \tau_{1}
\end{gathered}
$$

where

$$
A_{\zeta, \vec{\zeta}_{(m)}}^{(s)}(\mathbf{k}, \vec{k})=\chi_{\zeta, \vec{\zeta}}^{(s)}(\mathbf{k}, \vec{k}) M_{1}\left(\mathbf{k}^{\prime}\right) \ldots M_{s}\left(\mathbf{k}^{(s)}\right),
$$

$M_{j}$ are submonomials of $M$. According to Corollary 5.2 with $\tau_{*}=\beta$

$$
\left\|F_{2}\right\|_{L_{1}} \leq 2 C_{\Xi}^{1+2 s} C_{\chi} \beta \prod_{j=1}^{s}\left\|M_{j}\right\|_{E} \leq \beta C_{\Xi}^{e+2 i} \tau_{*}^{i-1} C_{\chi} \prod_{j=1}^{m}\left\|\tilde{\mathbf{h}}_{l_{j}}\right\|_{E} \leq \beta C_{\chi} C_{\Xi}^{e+2 i} \tau_{* E}^{i-1} R^{m} .
$$

Now we estimate $F_{1}$. Since $M(\mathcal{F}, T)$ is CI, there are two SI submonomials $M_{j_{1}}$ and $M_{j_{2}}$ applied to $\left(\tilde{\mathbf{h}}_{l_{j_{1}}}\right)^{m_{1}}$ and $\left(\tilde{\mathbf{h}}_{l_{j_{2}}}\right)^{m_{2}}$ with $l_{j_{1}} \neq l_{j_{2}}$. Let us assume that $l_{j_{1}}=l_{1}, l_{j_{2}}=l_{s}$ (the general case can be easily reduced to it by a relabeling of variables). We denote

$$
\phi^{\prime}=\nabla_{\mathbf{k}^{\prime}} \phi_{\zeta, \vec{\zeta}}\left(\mathbf{k}, \vec{k}_{*}\right)=\nabla_{\mathbf{k}^{\prime}} \omega\left(\mathbf{k}_{* l_{1}}\right)-\nabla_{\mathbf{k}^{(s)}} \omega\left(\mathbf{k}_{* l_{s}}\right) \neq 0, \mathbf{p}=\boldsymbol{\phi}^{\prime} /\left|\boldsymbol{\phi}^{\prime}\right|
$$

By (5.84) and (5.43) we obtain

$$
\left|\mathbf{p} \cdot \nabla_{\mathbf{k}^{\prime}} \phi_{\zeta, \vec{\zeta}}\left(\mathbf{k}, \vec{k}_{*}\right)\right| \geq p_{0}>0 \text { for } \vec{k}=\vec{k}_{*}=\left(\mathbf{k}_{* l_{1}}, \ldots, \mathbf{k}_{* l_{s}}\right) .
$$


Note that

$$
\exp \left\{\mathrm{i} \phi_{\zeta, \vec{\zeta}}(\mathbf{k}, \vec{k}) \frac{\tau_{1}}{\varrho}\right\}=\frac{\varrho}{\mathbf{i p} \cdot \nabla_{\mathbf{k}^{\prime}} \phi_{\zeta, \vec{\zeta}}(\mathbf{k}, \vec{k}) \tau_{1}} \mathbf{p} \cdot \nabla_{\mathbf{k}^{\prime}} \exp \left\{\mathrm{i} \phi_{\zeta, \vec{\zeta}}(\mathbf{k}, \vec{k}) \frac{\tau_{1}}{\varrho}\right\} .
$$

Using this identity, (2.25) and integrating by parts the integral which defines $F_{1}$ in (5.88) we obtain

$$
\begin{gathered}
F_{1}=\int_{\beta}^{\tau} I\left(\mathbf{k}, \tau_{1}\right) d \tau_{1}, I\left(\mathbf{k}, \tau_{1}\right)=\int_{\mathbb{D}_{m}} \exp \left\{\mathrm{i} \phi_{\zeta, \vec{\zeta}}(\mathbf{k}, \vec{k}) \frac{\tau_{1}}{\varrho}\right\} A_{\zeta, \vec{\zeta}}^{(s)}(\mathbf{k}, \vec{k}) \tilde{\mathrm{d}}^{(s-1) d} \vec{k}= \\
-\int_{\mathbb{D}_{s}} \frac{\varrho \exp \left\{\mathrm{i} \phi_{\zeta, \vec{\zeta}}(\mathbf{k}, \vec{k}) \frac{\tau_{1}}{\varrho}\right\}}{\mathrm{i} \tau_{1}} \mathbf{p} \cdot \nabla_{\mathbf{k}^{\prime}} \frac{A_{\zeta, \vec{\zeta}}^{(s)}(\mathbf{k}, \vec{k})}{\nabla_{\mathbf{k}^{\prime}} \phi_{\zeta, \vec{\zeta}}(\mathbf{k}, \vec{k}) \cdot \mathbf{p}} \tilde{\mathrm{d}}^{(s-1) d} \vec{k} .
\end{gathered}
$$

From (5.56), Lemma 5.12 and Corollary 5.13 we see that in the integral $I\left(\mathbf{k}, \tau_{1}\right)$ the integrands are nonzero only if

$$
\left|\mathbf{k}^{(j)}-\zeta^{(j)} \mathbf{k}_{*}^{(j)}\right| \leq m_{j} \pi_{0} \beta^{1-\epsilon},\left|\mathbf{k}-\zeta \mathbf{k}_{*}\right| \leq m \pi_{0} \beta^{1-\epsilon}, m_{1}+\ldots+m_{s} \leq m
$$

where $\pi_{0} \leq 1$. Using the Taylor remainder estimate for $\phi_{\zeta, \vec{\zeta}}$ at $\vec{k}_{*}$ we obtain the inequality

$$
\left|\nabla_{\mathbf{k}^{\prime}} \phi_{\zeta, \vec{\zeta}}(\mathbf{k}, \vec{k})-\phi^{\prime}\right| \leq 3 m \beta^{1-\epsilon} C_{\omega, 2} \text { if (15.94) holds. }
$$

Suppose that $\beta$ is small and satisfies

$$
3 m \beta^{1-\epsilon} C_{\omega, 2} \leq \frac{p_{0}}{2}
$$

Condition (5.96) is satisfied for small $\beta$ if $m \leq m(\beta)$ as in (5.23). Using (5.95) we derive from (5.92), (5.96) and (5.56) that

$$
\left|\mathbf{p} \cdot \nabla_{\mathbf{k}^{\prime}} \phi_{\zeta, \vec{\zeta}}(\mathbf{k}, \vec{k})\right| \geq \frac{p_{0}}{2}>0 \text { if (15.94) holds. }
$$

Now we use (5.97) to estimate denominators, (5.68) to estimate second $\mathbf{k}^{\prime}$-derivatives of $\omega$ and (5.69) to estimate $\nabla_{\mathbf{k}^{\prime}} \chi$. We conclude that

$$
\begin{aligned}
\left|I\left(\mathbf{k}, \tau_{1}\right)\right| \leq C_{\Xi}^{2 s+1} \int_{\mathbb{D}_{s}}\left[\frac{\varrho}{\tau_{1} p_{0}}\left|\nabla_{\mathbf{k}^{\prime}} A_{\zeta, \vec{\zeta}}^{(s)}(\mathbf{k}, \vec{k})\right|+\frac{8 \varrho C_{\omega, 2}}{\tau_{1} p_{0}^{2}}\left|A_{\zeta, \vec{\zeta}_{(m)}}^{(s)}(\mathbf{k}, \vec{k})\right|\right] \tilde{\mathrm{d}}^{(s-1) d} \vec{k} \\
\leq \frac{\varrho}{\tau_{1} p_{0}}\left[\left\|\left(\nabla_{k^{\prime}}-\nabla_{k^{(s)}}\right) \chi^{(s)}(\mathbf{k}, \cdot)\right\|+\frac{8 C_{\omega, 2}}{p_{0}}\left\|\chi^{(m)}(\mathbf{k}, \cdot)\right\|\right] C_{\Xi}^{2 s+1} \prod_{j=1}^{s}\left\|M_{j}\right\|_{L_{1}}+ \\
\quad \frac{\varrho C_{\Xi}^{2 s+1}\left\|\chi^{(s)}(\mathbf{k}, \cdot)\right\|}{\tau_{1} p_{0}}\left[\prod_{j=2}^{s}\left\|M_{j}\right\|_{L_{1}}\left\|\nabla_{\mathbf{k}^{\prime}} M_{1}\right\|_{L_{1}}+\prod_{j=1}^{s-1}\left\|M_{j}\right\|_{L_{1}}\left\|\nabla_{\mathbf{k}^{(s)}} M_{s}\right\|_{L_{1}}\right] .
\end{aligned}
$$


To estimate $\nabla M_{i}$ we use Lemma [5.21, We also use (5.2) and (5.5) to estimate $\left\|M_{j}\right\|_{L_{1}}$. Therefore, using (5.72), we obtain

$$
\left|I\left(\mathbf{k}, \tau_{1}\right)\right| \leq \frac{C}{\tau_{1}} \tau_{*}^{i-1} C_{\Xi}^{2 i+e} C_{\chi}^{i} \frac{\varrho}{\beta^{1+\epsilon} p_{0}} m^{2} R^{m-1}
$$

Finally, we consider the case when the alternative Condition 2.23 holds. In this case $M_{1}$ and $M_{s}$ according to Lemma 5.12 are localized near $\nu_{1} \mathbf{k}_{* l_{1}}$ and $\nu_{2} \mathbf{k}_{* l_{s}}$ with some $\nu_{1}$ and $\nu_{2}$; we use (2.94) to obtain (5.92) both for AFM and ANFM submonomials. Therefore (5.97) holds and we again get (5.98) and (5.99). So, we proved (5.99) in all cases. Integrating (5.99) in $\tau_{1}$ we obtain

$$
\left\|F_{1}\right\|_{E} \leq C \tau_{*}^{i-1} C_{\Xi}^{2 i+e} C_{\chi}^{i} \frac{\varrho}{\beta^{1+\epsilon} p_{0}} m^{2}|\ln \beta| R^{m-1} .
$$

Using summation over all $\vec{\lambda}, \vec{\zeta}$ (the sum involves no more than $3^{2 m}$ terms) we obtain (5.85) from (5.87) and (5.100).

\section{Proof of the superposition theorems}

In this section we prove Theorems 2.15, 2.19 on the approximate modal superposition principle.

\subsection{Proof of the Superposition principle for lattice equations}

Here we prove Theorem 2.15] First we note that according to Lemma 5.6] we can replace $\tilde{\mathbf{h}}_{l}$ by $\tilde{\mathbf{h}}_{l}^{\Psi}$ in the statement of Theorem 2.15) in particular in (2.47), (2.48). Hence we can assume that (5.56) holds.

Based on Theorem 5.4 we expand the solution of (2.3) into series (5.8) and then into the sum of composition monomials $M(\mathcal{F}, T)$ as in (4.33):

$$
\mathcal{G}(\mathcal{F}, \tilde{\mathbf{h}})=\tilde{\mathbf{h}}+\sum_{m=2}^{\infty} \sum_{T \in T_{m}} c_{T} M(\mathcal{F}, T)\left(\tilde{\mathbf{h}}^{m}\right)
$$

where

$$
\tilde{\mathbf{h}}=\sum_{l=1}^{N_{h}} \tilde{\mathbf{h}}_{l},\left\|\tilde{\mathbf{h}}_{l}\right\|_{E} \leq R, l=1, \ldots, N_{h},
$$

and the relation (5.19) (that is $N_{h} R<R_{\mathcal{G}}$ ) holds, where $R_{\mathcal{G}}$ is the radius of convergence from Theorem 5.4. $R$ will be specified below. Using Lemma 5.7 we conclude that

$$
\mathcal{G}(\mathcal{F}, \tilde{\mathbf{h}})=\tilde{\mathbf{h}}+\sum_{m=2}^{m(\beta)} \sum_{T \in T_{m}} c_{T} M(\mathcal{F}, T)\left(\tilde{\mathbf{h}}^{m}\right)+g,\|g\|_{E} \leq \beta
$$


where $m(\beta)$ is defined by (15.23) . Then we expand every monomial $M(\mathcal{F}, T)\left(\tilde{\mathbf{h}}^{m}\right)$ according to (5.28) into the sum of the terms $M(\mathcal{F}, T)\left(\tilde{\mathbf{h}}_{l_{1}} \ldots \tilde{\mathbf{h}}_{l_{m}}\right)$. Note that since $m(\beta) \leq C|\ln \beta|$, conditions (5.96), (5.70), (5.47) are satisfied if $\beta$ is small enough for every $m \leq m(\beta)$. The monomials $M(\mathcal{F}, T)\left(\tilde{\mathbf{h}}_{l_{1}} \ldots \tilde{\mathbf{h}}_{l_{m}}\right)$ belong to two classes, SI and CI (according to Definition 5.8) and the class is determined by the multiindex $\left(l_{1}, \ldots, l_{m}\right)=\bar{l}$. Using (6.3) we conclude that

$$
\begin{gathered}
\mathcal{G}\left(\mathcal{F}, \sum_{l=1}^{N_{h}} \tilde{\mathbf{h}}_{l}\right)=\sum_{l=1}^{N_{h}} \mathcal{G}\left(\mathcal{F}, \tilde{\mathbf{h}}_{l}\right)+\tilde{\mathbf{D}}, \\
\tilde{\mathbf{D}}=\sum_{m=2}^{m(\beta)} \sum_{T \in T_{m}} \sum_{\mathrm{CI}} c_{T} M(\mathcal{F}, T)\left(\tilde{\mathbf{h}}_{l_{1}, \ldots, l_{m}} \ldots \tilde{\mathbf{h}}_{l_{m}}\right)+g_{1},\left\|g_{1}\right\|_{E} \leq C \beta .
\end{gathered}
$$

To obtain (2.48) we have to estimate the sum in $\tilde{\mathbf{D}}$ and show that it is small. It follows from (4.35) that

$$
\begin{gathered}
\left\|\sum_{m=2}^{m(\beta)} \sum_{T \in T_{m}} \sum_{\mathrm{Cl} l_{1}, \ldots, l_{m}} c_{T} M(\mathcal{F}, T)\left(\tilde{\mathbf{h}}_{l_{1}} \ldots \tilde{\mathbf{h}}_{l_{m}}\right)\right\|_{E} \leq \\
\leq \sum_{m=2}^{m(\beta)} N_{h}^{m} \sum_{T \in T_{m}} c_{T} \sup _{T \in T_{m}, \mathrm{CI} \bar{l}}\left\|M(\mathcal{F}, T)\left(\tilde{\mathbf{h}}_{l_{1}} \ldots \tilde{\mathbf{h}}_{l_{m}}\right)\right\|_{E} \leq \\
\leq \sum_{m=2}^{m(\beta)} N_{h}^{m} c_{0} c_{1}^{m} \sup _{T \in T_{m}, \mathrm{CI} \bar{l}}\left\|M(\mathcal{F}, T)\left(\tilde{\mathbf{h}}_{l_{1}} \ldots \tilde{\mathbf{h}}_{l_{m}}\right)\right\|_{E} \cdot
\end{gathered}
$$

Now we consider an evaluated monomial $M(\mathcal{F}, T)\left(\tilde{\mathbf{h}}_{l_{1}} \ldots \tilde{\mathbf{h}}_{l_{m}}\right)$ with arguments given by CI multiindex $\bar{l}=\left(l_{1}, \ldots, l_{m}\right)$. To prove that this monomial has a small norm, according to Lemma 4.24 it is sufficient to show that one of its submonomials is small and the relevant operators are bounded. According to Proposition [5.9] the monomial $M(\mathcal{F}, T)\left(\tilde{\mathbf{h}}_{l_{1}} \ldots \tilde{\mathbf{h}}_{l_{m}}\right)$ contains a submonomial $M\left(\mathcal{F}, T^{\prime}\right)\left(\tilde{\mathbf{h}}_{l_{s^{\prime}}} \ldots \tilde{\mathbf{h}}_{l_{s^{\prime \prime}}}\right)$ with the homogeneity index $s=s^{\prime \prime}-s^{\prime}+1$, the incidence number $i^{\prime}$ and the rank $r^{\prime}$ which is minimal in the following sense. The monomial $M\left(\mathcal{F}, T^{\prime}\right)\left(\tilde{\mathbf{h}}_{l_{s^{\prime}}} \ldots \tilde{\mathbf{h}}_{l_{s^{\prime \prime}}}\right)$ is CI, but every its submonomial $M\left(\mathcal{F}, T^{\prime \prime}\right)\left(\tilde{\mathbf{h}}_{l_{s^{\prime \prime}}} \ldots \tilde{\mathbf{h}}_{l_{s^{\prime \prime \prime}}}\right)$ is SI. Now we use the space decomposition (5.1) and expand $M\left(\mathcal{F}, T^{\prime}\right)$ as in (4.44) into a sum of no more than $3^{2 m}$ decorated monomials $M\left(\mathcal{F}, T^{\prime}, \vec{\lambda}, \vec{\zeta}\right)\left(\tilde{\mathbf{h}}_{l_{s^{\prime}}} \ldots \tilde{\mathbf{h}}_{l_{s^{\prime \prime}}}\right)$. The decorated submonomials of every decorated monomial are SI. We apply Lemma 5.22 and conclude that

$$
\left\|M\left(\mathcal{F}, T^{\prime}, \vec{\lambda}, \vec{\zeta}\right)\left(\tilde{\mathbf{h}}_{l_{s^{\prime}}} \ldots \tilde{\mathbf{h}}_{l_{s^{\prime \prime}}}\right)\right\|_{E} \leq C\left[\frac{\varrho}{\beta^{1+\epsilon}}|\ln \beta|+\beta\right] \frac{s^{2}}{p_{0}} \tau_{*}^{i^{\prime}-1} C_{\Xi}^{e^{\prime}+2 i^{\prime}} C_{\chi}^{i^{\prime}} R^{s^{\prime \prime}-s^{\prime}} .
$$


Hence, there is a submonomial of $M(\mathcal{F}, T)\left(\tilde{\mathbf{h}}_{l_{1}} \ldots \tilde{\mathbf{h}}_{l_{m}}\right)$ with a small norm. Namely, since (2.46) and (2.5) are assumed, this small submonomial provides the smallness of the norm of the whole monomial $M(\mathcal{F}, T)\left(\tilde{\mathbf{h}}_{l_{1}} \ldots \tilde{\mathbf{h}}_{l_{m}}\right)$ according to Lemma 4.24. We also use Corollary 5.2 and (2.26) to estimate norms of remaining submonomials of rank $r$ and apply (4.32) and (5.72) to obtain

$$
\left\|M(\mathcal{F}, T)\left(\tilde{\mathbf{h}}_{l_{1}} \ldots \tilde{\mathbf{h}}_{l_{m}}\right)\right\| \leq 3^{2 m}\left[\frac{\varrho}{\beta^{1+\epsilon}}|\ln \beta|+\beta\right] C_{1} m^{2} \tau_{*}^{i-1} C_{\Xi}^{e+2 i} C_{\chi}^{i} R^{m-1} .
$$

Since $e=i+m-1$, using (4.25) and the inequalities $i(T)=i \geq m / m_{F}, i \leq m-1$ we get

$$
\begin{aligned}
& \sum_{m=2}^{m(\beta)} \sum_{T \in T_{m}} \sum_{C I l_{1}, \ldots, l_{m}} c_{T} M(\mathcal{F}, T)\left(\tilde{\mathbf{h}}_{l_{1}} \ldots \tilde{\mathbf{h}}_{l_{m}}\right) \\
\leq & C_{2}\left[\frac{\varrho}{\beta^{1+\epsilon}}|\ln \beta|+\beta\right] \sum_{m=2}^{\infty} \tau_{*}^{m / m_{F}-1} m^{2} N_{h}^{m} c_{1}^{m} R^{m-1}
\end{aligned}
$$

with $c_{1}=9 C_{\Xi}^{5} C_{\chi}$. The series converges if, in addition to (5.19), $R$ satisfies the inequality

$$
R N_{h} c_{1} \tau_{*}^{1 / m_{F}}<1
$$

For such $R$ and $\tau_{*}$, combining (6.7) with (6.3) and using (2.46) we obtain (2.48), and the Theorem 2.15 is proved.

\subsection{Proof of the Superposition principle for PDE}

Here we prove Theorem 2.25 (and its particular case Theorem 2.19). The proof is completely similar to the above proof of Theorem 2.15 up to every detail. One only have to replace $\mathbb{D}_{m}$ given by (2.23) by $\mathbb{D}_{m}$ given by (2.65) and the space $L_{1}$ is now defined by (2.66) instead of (2.31).

Remark 6.1 Note that smallness of CI terms is essential and is based on different group velocities of single band wavepackets. Note that separation of different wavepackets based only on FM and NFM arguments as in Lemma 5.17 is impossible since there are always FM monomials with different $l$ because of the symmetry conditions (2.15), (2.16), for example FM condition

$$
\zeta \omega_{n, \zeta}\left(\zeta \mathbf{k}_{*}\right)-\zeta^{\prime} \omega_{n^{\prime}}\left(\zeta^{\prime} \mathbf{k}_{* 1}\right)-\zeta^{\prime \prime} \omega_{n^{\prime \prime}}\left(\zeta^{\prime \prime} \mathbf{k}_{* 2}\right)-\zeta^{\prime \prime \prime} \omega_{n^{\prime \prime \prime}}\left(\zeta^{\prime \prime \prime} \mathbf{k}_{* 3}\right)=0
$$

is fulfilled if

$$
n=n^{\prime}, \zeta=\zeta^{\prime}, \mathbf{k}_{*}=\mathbf{k}_{* 1}, n^{\prime \prime}=n^{\prime \prime \prime}, \zeta^{\prime \prime}=-\zeta^{\prime \prime \prime}, \mathbf{k}_{* 2}=\mathbf{k}_{* 3}
$$

independently of the values of $\mathbf{k}_{*}, \mathbf{k}_{* 3}$ and independently of a particular form of functions $\omega_{n}(\mathbf{k})$. 


\section{Examples and possible generalizations}

\subsection{Fermi-Pasta-Ulam equation}

FPU equation on the infinite lattice has the form

$$
\begin{gathered}
\partial_{t}^{2} x_{n}=\left(x_{n-1}-2 x_{n}+x_{n+1}\right)+\alpha_{3}\left(\left(x_{n+1}-x_{n}\right)^{3}-\left(x_{n}-x_{n-1}\right)^{3}\right) \\
+\alpha_{2}\left(\left(x_{n+1}-x_{n}\right)^{2}-\left(x_{n}-x_{n-1}\right)^{2}\right) .
\end{gathered}
$$

It can be reduced to the following first-order equation

$$
\partial_{t} x_{n}=y_{n}-y_{n-1}, \partial_{t} y_{n}=x_{n+1}-x_{n}+\alpha_{3}\left(x_{n+1}-x_{n}\right)^{3}+\alpha_{2}\left(x_{n+1}-x_{n}\right)^{2} .
$$

We introduce lattice Fourier transforms $\tilde{x}(k)$ and $\tilde{y}(k)$ by (2.2), namely

$$
\tilde{x}(k)=\sum_{n} x_{n} \mathrm{e}^{-\mathrm{i} n k}, k \in[-\pi, \pi] .
$$

First we write Fourier transform of the linear part of (7.2) (that is with $\alpha_{3}=\alpha_{2}=0$ ). Multiplying by $\mathrm{e}^{-\mathrm{i} n k}$ and doing summation we obtain

$$
\partial_{t} \tilde{x}(k)=\tilde{y}(k)-\mathrm{e}^{-\mathrm{i} k} \tilde{y}(k), \partial_{t} \tilde{y}(k)=\mathrm{e}^{\mathrm{i} k} \tilde{x}(k)-\tilde{x}(k) .
$$

that can be recast in the matrix form as follows

$$
\partial_{t}\left[\begin{array}{l}
\tilde{x} \\
\tilde{y}
\end{array}\right]=\left[\begin{array}{cc}
0 & -\left(\mathrm{e}^{\mathrm{i} k}-1\right)^{*} \\
\mathrm{e}^{\mathrm{i} k}-1 & 0
\end{array}\right]\left[\begin{array}{l}
\tilde{x} \\
\tilde{y}
\end{array}\right] .
$$

The eigenvalues of the matrix are purely imaginary and equal $i \omega_{\zeta}(k)$ with

$$
\omega_{\zeta}(k)=\zeta\left|\mathrm{e}^{\mathrm{i} k}-1\right|=2 \zeta\left|\sin \frac{k}{2}\right|, \zeta= \pm,-\pi \leq k \leq \pi .
$$

The eigenvectors are orthogonal and are given explicitly by

$$
\mathbf{g}_{\zeta}(k)=\frac{1}{\sqrt{2}\left|\mathrm{e}^{\mathrm{i} k}-1\right|}\left[\begin{array}{c}
i \zeta\left|\mathrm{e}^{\mathrm{i} k}-1\right| \\
\mathrm{e}^{\mathrm{i} k}-1
\end{array}\right]=\frac{1}{\sqrt{2}}\left[\begin{array}{c}
i \zeta \\
\frac{\mathrm{e}^{\mathrm{i} k}-1}{\left|\mathrm{e}^{\mathrm{i} k}-1\right|}
\end{array}\right], \zeta= \pm, k \neq 0 .
$$

Now let us consider nonlinear terms. Note that the lattice Fourier transform of the product $x(\mathbf{n}) z(\mathbf{n}), \mathbf{n} \in \mathbb{Z}^{d}$ is given by the following convolution formula

$$
\widetilde{x z}(\mathbf{k})=\frac{1}{(2 \pi)^{d}} \int_{[-\pi, \pi]^{d}} \tilde{x}(\mathbf{s}) \tilde{z}(\mathbf{k}-\mathbf{s}) \mathrm{d} \mathbf{s}
$$

as in the case of the continuous Fourier transform. Note that

$$
\widetilde{x_{n+1}-} x_{n}(k)=\left(\mathrm{e}^{\mathrm{i} k}-1\right) \tilde{x}(k),
$$


and, hence, the Fourier transform of the cubic term of the nonlinearity in (17.2) is

$$
=\frac{1}{(2 \pi)^{2}} \int_{\substack{k^{\prime}+k^{\prime \prime}+k^{\prime \prime \prime}=\mathbf{k} \\[-\pi, \pi]^{2}}}\left(\mathrm{e}^{\left.\mathrm{i} k^{\prime}-1\right)}\left(\mathrm{e}^{\mathrm{i} k^{\prime \prime}}-1\right)\left(\mathrm{e}^{\mathrm{i} k^{\prime \prime \prime}}-1\right) \tilde{x}\left(k^{\prime}\right) \tilde{x}\left(k^{\prime \prime}\right) \tilde{x}\left(k^{\prime \prime \prime}\right) \mathrm{d} k^{\prime} \mathrm{d} k^{\prime \prime},\right.
$$

and similar convolution for the quadratic term.

\subsection{Examples of wavepacket data}

Here we give examples of initial data for PDE in $\mathbb{R}^{d}$ and on the lattice $\mathbb{Z}^{d}$ which are wavepackets in the sense of Definition [2.9, We define a wavepacket by (2.33) where $h_{\zeta}$ is chosen to satisfy (2.35) and (2.34).

Recall that a Schwartz function is an infinitely smooth function $\Phi(\mathbf{r}), \mathbf{r} \in \mathbb{R}^{d}$ which rapidly decays and satisfies for every $s \geq 0$ the inequality

$$
\sup _{\mathbf{r}} \sum_{|\alpha|+p \leq s}|\mathbf{r}|^{p}\left|\partial_{\mathbf{r}}^{\alpha} \Phi(\mathbf{r})\right| d \mathbf{r} \leq C_{1}(s)
$$

where

$$
\partial_{\mathbf{r}}^{\alpha} \Phi(\mathbf{r})=\partial_{r_{1}}^{\alpha_{1}} \ldots \partial_{r_{d}}^{\alpha_{d}} \Phi(\mathbf{r}), \alpha=\left(\alpha_{1}, \ldots, \alpha_{d}\right),|\alpha|=\alpha_{1}+\ldots+\alpha_{d} .
$$

It is well known that Fourier transform of a Schwartz function remains to be a Schwartz function and that its derivatives satisfy the inequality

$$
\left.\sup _{\mathbf{k}} \sum_{|\alpha|+p \leq s}|| \mathbf{k}\right|^{p} \partial_{\mathbf{k}}^{\alpha} \hat{\Phi}(\mathbf{k}) \mid \leq C_{2}(s) .
$$

Example 1. We consider equation in $\mathbb{R}^{d}$ as in Subsection 1.2. The simplest example of a wavepacket in the sense of Definition 2.9 is a function of the form (2.36) where

$$
\int_{\mathbb{R}^{d}}\left|\hat{h}_{\zeta}(\mathbf{k})\right|+\left|\nabla_{\mathbf{k}} \hat{h}_{\zeta}(\mathbf{k})\right|+|\mathbf{k}|^{1 / \epsilon}\left|\hat{h}_{\zeta}(\mathbf{k})\right| \mathrm{d} \mathbf{k}<\infty, .
$$

and $\mathbf{g}_{n, \zeta}(\mathbf{k})$ is an eigenvector from (2.13). Note that $\beta^{-d} \hat{h}_{\zeta}(\mathbf{k} / \beta)$ is the Fourier transform of a function $h_{\zeta}(\beta \mathbf{r})$.

Lemma 7.1 Let $\hat{\mathbf{h}}(\beta, \mathbf{k}), \mathbf{k} \in \mathbb{R}^{d}$ be defined by (2.36), (7.8). Then $\hat{\mathbf{h}}_{l, \zeta}(\beta, \mathbf{k})$ is a wavepacket with wavepacket center $\mathbf{k}_{*}$ in the sense of Definition 2.9 with $L_{1}=L_{1}\left(\mathbb{R}^{d}\right)$.

Proof. First, condition (2.32) holds since

$$
\left\|\hat{\mathbf{h}}_{\zeta}(\beta, \cdot)\right\|_{L_{1}}=\int_{\mathbb{R}^{d}} \beta^{-d}\left|\hat{h}_{\zeta}\left(\frac{\mathbf{k}-\zeta \mathbf{k}_{*}}{\beta}\right) \mathbf{g}_{n, \zeta}\left(\mathbf{k}_{*}\right)\right| \mathrm{d} \mathbf{k}=\left|\mathbf{g}_{n, \zeta}\left(\mathbf{k}_{*}\right)\right| \int_{\mathbb{R}^{d}}\left|\hat{h}_{\zeta}(\mathbf{k})\right| \mathrm{d} \mathbf{k} .
$$


Condition (2.33) is obviously fulfilled since

$$
\hat{\mathbf{h}}_{\zeta}(\beta, \mathbf{k})=\Pi_{n, \zeta}(\mathbf{k}) \tilde{\mathbf{h}}_{\zeta}(\beta, \mathbf{k}) .
$$

Inequality (2.34) follows from the estimate

$$
\beta^{-d} \int_{\left|\mathbf{k}-\zeta \mathbf{k}_{*}\right| \geq \beta^{1-\epsilon}}\left|\hat{h}_{\zeta}\left(\frac{\mathbf{k}-\zeta \mathbf{k}_{*}}{\beta}\right)\right| \mathrm{d} \mathbf{k} \leq \beta \int_{|\mathbf{k}| \geq \beta^{-\epsilon}}|\mathbf{k}|^{1 / \epsilon}\left|\hat{h}_{\zeta}(\mathbf{k})\right| \mathrm{d} \mathbf{k} \leq C \beta .
$$

To verify (2.35) we note that since $\Pi_{n, \zeta}(\mathbf{k})$ smoothly depend on $k$ near $\zeta \mathbf{k}_{*}$ we have

$$
\begin{gathered}
\int_{\left|\mathbf{k}-\zeta \mathbf{k}_{*}\right| \leq \beta^{1-\epsilon}}\left|\nabla_{\mathbf{k}} \hat{\mathbf{h}}_{\zeta}(\beta, \mathbf{k})\right| \mathrm{d} \mathbf{k} \\
\leq C \int_{\left|\mathbf{k}-\zeta \mathbf{k}_{*}\right| \leq \beta^{1-\epsilon}} \beta^{-d-1}\left|\nabla_{\mathbf{k}} \hat{h}_{l}\left(\frac{\mathbf{k}-\zeta \mathbf{k}_{*}}{\beta}\right)\right|+\beta^{-d}\left|\hat{h}_{l}\left(\frac{\mathbf{k}-\zeta \mathbf{k}_{*}}{\beta}\right)\right| \mathrm{d} \mathbf{k} \\
\leq C \beta^{-1} \int_{\mathbb{R}^{d}}\left|\nabla_{\mathbf{k}} \hat{h}_{\zeta}(\mathbf{k})\right| \mathrm{d} \mathbf{k}+C
\end{gathered}
$$

and (7.8) implies (2.35).

Example 2. Let us consider a lattice equation in $\mathbb{Z}^{d}$ as in Section 1.1. We would like to give a sufficient condition for functions defined on the lattice which ensures that their Fourier transforms satisfy all requirements of Definition 2.9. We pick a Schwartz function $\Phi(\mathbf{r}$ ) (see (17.6)), a vector $\mathbf{k}_{*} \in[-\pi, \pi]^{d}$ and introduce

$$
h(\beta, \mathbf{r})=\mathrm{e}^{-\mathrm{i} \mathbf{r} \cdot \mathbf{k}_{*}} \Phi(\beta \mathbf{r}), \mathbf{r} \in \mathbb{R}^{d} .
$$

Then we restrict the above function to the lattice $\mathbb{Z}^{d}$ by setting $\mathbf{r}=\mathbf{m}$. The following lemma is similar to Lemma 7.1 .

Lemma 7.2 Let $\Phi(\mathbf{r})$ be a Schwartz function, $h_{\zeta}(\beta, \mathbf{r})$ be defined by 7.10$), \tilde{h}_{\zeta}(\beta, \mathbf{k})$ be its lattice Fourier transform. Then the function $\tilde{h}_{\zeta}(\beta, \mathbf{k})$ extended to $\mathbb{R}^{d}$ as a periodic function with period $2 \pi$ satisfies all requirements of Definition 2.9 with $L_{1}=L_{1}\left([-\pi, \pi]^{d}\right)$.

Proof. The lattice Fourier transform of $h(\beta, \mathbf{r})$ equals

$$
\tilde{h}(\beta, \mathbf{k})=\sum_{\mathbf{m} \in \mathbb{Z}^{d}} \mathrm{e}^{-\mathbf{i m} \cdot \mathbf{k}_{*}} \Phi(\beta \mathbf{m}) e^{-\mathbf{i m} \cdot \mathbf{k}}=\sum_{\mathbf{m} \in \mathbb{Z}^{d}} \Phi(\beta \mathbf{m}) \mathrm{e}^{-\mathrm{im} \cdot\left(\mathbf{k}-\mathbf{k}_{*}\right)} .
$$

Since the above expression naturally defines $\tilde{h}(\beta, \mathbf{k})$ as a function of $\mathbf{k}-\mathbf{k}_{*}$, it is sufficient to take $\mathbf{k}_{*}=0$. To get (2.34), we use the representation of $\Phi(\mathbf{r})$ in terms of inverse Fourier transform (2.60)

$$
\Phi(\mathbf{r})=\frac{1}{(2 \pi)^{d}} \int_{\mathbb{R}^{d}} \hat{\Phi}(\mathbf{k}) \mathrm{e}^{\mathrm{i} \mathbf{r} \cdot \mathbf{k}} \mathrm{d} \mathbf{k}, \Phi(\beta \mathbf{m})=\frac{1}{(2 \pi \beta)^{d}} \int_{\mathbb{R}^{d}} \hat{\Phi}\left(\frac{1}{\beta} \mathbf{k}\right) \mathrm{e}^{\mathrm{i} \mathbf{m} \cdot \mathbf{k}} \mathrm{d} \mathbf{k} .
$$


We split $\Phi(\beta \mathbf{m})$ into two terms:

$$
\begin{aligned}
& \Phi(\beta \mathbf{m})=\frac{1}{(2 \pi \beta)^{d}} \int_{\mathbb{R}^{d}} \Psi\left(\frac{1}{\beta^{1-\epsilon}} \xi\right) \hat{\Phi}\left(\frac{1}{\beta} \xi\right) \mathrm{e}^{\mathrm{i} \mathbf{m} \cdot \boldsymbol{\xi}} \mathrm{d} \boldsymbol{\xi}+\Phi_{1}(\mathbf{m}) \\
& \Phi_{1}(\mathbf{m})=\frac{1}{(2 \pi \beta)^{d}} \int_{\mathbb{R}^{d}}\left(1-\Psi\left(\frac{1}{\beta^{1-\epsilon}} \xi\right)\right) \hat{\Phi}\left(\frac{1}{\beta} \xi\right) \mathrm{e}^{\mathrm{i} \mathbf{m} \cdot \boldsymbol{\xi}} \mathrm{d} \boldsymbol{\xi}
\end{aligned}
$$

with $\Psi(\xi)$ defined by (5.12). The first term in (7.13) coincides with the inverse lattice Fourier transform, its lattice Fourier transform is explicitly given and can be treated as in Lemma [7.1. The second term gives $O\left(\beta^{N}\right)$ with large $N$ for Schwartz functions $\hat{\Phi}$. Using these observations we check all points of Definition [2.9] as in Lemma [7.1]

\subsection{The Nonlinear Maxwell equation}

We expect that the approximate superposition principle can be generalized to the Nonlinear Maxwell equations (NLM) in periodic media studied in 4]. A concise operator form of the NLM is

$$
\partial_{\tau} \mathbf{U}=-\frac{\mathrm{i}}{\varrho} \mathbf{M U}+\mathcal{F}_{\mathrm{NL}}(\mathbf{U})-\mathbf{J}_{0}, \mathbf{U}(\tau)=0 \text { for } \tau \leq 0,
$$

where the excitation current

$$
\mathbf{J}(\tau)=0 \text { for } \tau \leq 0 .
$$

We were studying the properties of nonlinear wave interactions as described by the Nonlinear Maxwell equations in series of papers [1]- 6]. Our analysis of the solutions to the NLM uses an expansion in terms of orthonormal Floquet-Bloch basis $\tilde{\mathbf{G}}_{n, \zeta}(\mathbf{r}, \mathbf{k}), n=1, \ldots$, namely

$$
\tilde{\mathbf{U}}(\mathbf{k}, \mathbf{r}, \tau)=\sum_{\zeta= \pm 1} \sum_{n=1}^{\infty} \tilde{U}_{n, \zeta}(\mathbf{k}, \tau) \tilde{\mathbf{G}}_{n, \zeta}(\mathbf{r}, \mathbf{k}), \mathbf{k} \in[-\pi, \pi]^{d}
$$

This expansion is similar to (2.18) with $J$ replaced by $\infty$, since the linear Maxwell operator in a periodic medium has infinitely many bands. The excitation currents take the form similar to forcing term in (3.1), namely

$$
\begin{gathered}
\tilde{\mathbf{J}}(\mathbf{r}, \mathbf{k}, \tau)=\tilde{j}_{n,+}(\mathbf{k}, \tau) \tilde{\mathbf{G}}_{n,+}(\mathbf{r}, \mathbf{k}) \mathrm{e}^{-\frac{i}{\varrho} \omega_{n}(\mathbf{k}) \tau}+\tilde{j}_{n,-}(\mathbf{k}, \tau) \tilde{\mathbf{G}}_{n,-}(\mathbf{r}, \mathbf{k}) \mathrm{e}^{\frac{\mathrm{i}}{\varrho} \omega_{n}(\mathbf{k}) \tau} \\
\tilde{\mathbf{J}}_{n}(\mathbf{r}, \mathbf{k}, \tau)=0, n \neq n_{0}
\end{gathered}
$$

with a fixed $n=n_{0}$. The difference with (3.1) is that time-independent $\mathbf{h}_{n, \zeta}(\mathbf{k})$ is replaced by $\tilde{j}_{n, \zeta}(\mathbf{k}, \tau)$. The functions $\tilde{j}_{n, \zeta}(\mathbf{k}, \tau)$ for every $\tau$ have the form of wavepackets in the sense of Definition 2.9, or in particular the form similar to (2.36) with fixed $\mathbf{k}_{*}$.

The Existence and uniqueness Theorem for the NLM is proven in 4], in particular function-analytic representation of the solution as a function of the excitation current. The results of this paper can be extended to the NLM equations provided that certain technical 
difficulties are addressed. Particularly, the classical NLM equation allows for the time dispersion with consequent time-convolution integration in the nonlinear term. This complication can be addressed by approximating it with a nonlinearity of the form (2.22) with an error $O(\varrho)=O\left(\beta^{2}\right)$, see $[6$. Then the derivation of the approximate linear superposition principle for wavepackets can be done as in this paper. Another complication with the NLM is that it has infinite number of bands.

\subsection{Dissipative terms in the linear part}

Equations (2.3) and (2.61) involve linear operators iL (k) with purely imaginary spectrum. Quite similarly we can consider equations of the form

$$
\partial_{\tau} \hat{\mathbf{U}}(\mathbf{k}, \tau)=\left[-\mathbf{G}(\mathbf{k})-\frac{\mathrm{i}}{\varrho} \mathbf{L}(\mathbf{k})\right] \hat{\mathbf{U}}(\mathbf{k}, \tau)+\hat{F}(\hat{\mathbf{U}})(\mathbf{k}, \tau),
$$

where a Hermitian matrix $\mathbf{G}(\mathbf{k})$ commutes with the Hermitian matrix $\mathbf{L}(\mathbf{k})$ and $\mathbf{G}(\mathbf{k})$ is nonnegative. In this case the approximate superposition principle also holds. The proofs are quite similar. In the case (2.61), which corresponds to of PDE, G $(\mathbf{k})$ determines a dissipative term, for example $\mathbf{G}(\mathbf{k})=|\mathbf{k}|^{2} I, \mathbf{k} \in \mathbb{R}^{d}$, where $I$ is the identity matrix, corresponds to Laplace operator $\Delta$. When such a dissipative term is introduced, we can consider nonlinearities $\hat{F}$ which involve derivatives, see [8], 9] in a similar situation. For such nonlinearities our framework remains the same, but some statements and proofs have to be modified. We will consider this case in a separate paper.

\section{Appendix A: Structure of a composition monomial based on oscillatory integrals}

Every composition monomial $M\left(\mathcal{F}, T, \vec{\lambda}_{(\hat{s})}, \vec{\zeta}_{(m)}\right)\left(\tilde{\mathbf{h}}_{1} \ldots \tilde{\mathbf{h}}_{m}\right)$ based on oscillatory integral operators $\mathcal{F}^{(m)}$ as defined by (3.14) and the space decomposition as defined by (5.1) has the following structure. Let $T$ be the tree corresponding to the monomial $M$. The monomial involves integration with respect to time variables $\tau_{(N)}$ where $N \in T$ are the nodes of the tree $T$. The monomial also involves integration with respect to variables $\mathbf{k}_{N}, N \in T$. The argument of the integral operator $M\left(\mathcal{F}, T, \vec{\lambda}_{(\hat{s})}, \vec{\zeta}_{(m)}\right)$ involves only end nodes (of zero rank) and has the form

$$
\prod_{\operatorname{rank}(N)=0} \tilde{\mathbf{h}}_{N}\left(\mathbf{k}_{N}\right)
$$

The kernel of the integral operator involves the composition monomial $M\left(\chi, T, \vec{\lambda}_{(\hat{s})}, \vec{\zeta}_{(m)}\right)$

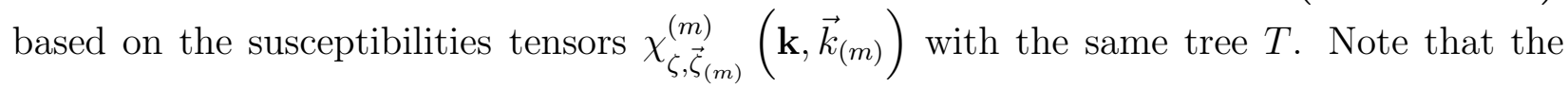


phase matching condition (3.12) takes the form

$$
\mathbf{k}_{N}=\mathbf{k}_{N}^{\prime}+\ldots+\mathbf{k}_{N}^{(\mu(N))}=\sum_{i=1}^{\mu(N)} \mathbf{k}_{c_{i}(N)} .
$$

Recall that if $c_{i}(N), i=1, \ldots, \mu(N)$ is the $i$-th child node of $N$, then the arguments in (3.14) are determined by the formula

$$
\mathbf{k}_{c_{i}(N)}=\mathbf{k}_{N}^{\left(c_{i}\right)}
$$

Hence, the kernel of the integral operator $M\left(\mathcal{F}, T, \vec{\lambda}_{(\hat{s})}, \vec{\zeta}_{(m)}\right)\left(\tilde{\mathbf{h}}_{1} \ldots \tilde{\mathbf{h}}_{m}\right)$ involves the product of normalized delta functions

$$
\prod_{\operatorname{rank}(N)>0} \delta\left(\mathbf{k}_{N}-\mathbf{k}_{c_{1}(N)}-\ldots-\mathbf{k}_{c_{\mu(N)}(N)}\right)
$$

and the integration with respect to $\mathbf{k}_{N}$ is over the torus

$$
\left(\prod_{N \neq N_{*}} \int_{[-\pi, \pi]^{\mu(N) d}}\right)[\cdots] \prod_{N \neq N_{*}} \mathrm{~d} \mathbf{k}_{N}
$$

and, obviously, the variable $\mathbf{k}_{N_{*}}$ corresponding to the root node $N_{*}$ is not involved into the integration.

Since every operator $\mathcal{F}^{(m)}$ at a node $N$ of the monomial $M\left(\mathcal{F}, T, \vec{\lambda}_{(\hat{s})}, \vec{\zeta}_{(m)}\right)$ contains the oscillatory factor

$$
\begin{aligned}
& \exp \left\{\mathrm{i} \phi_{\zeta, \vec{\zeta}_{(m), N}}\left(\mathbf{k}, \vec{k}_{(m)}\right) \frac{\tau_{(N)}}{\varrho}\right\}= \\
& \exp \left\{\mathrm{i}\left[\zeta_{N} \omega\left(\mathbf{k}_{N}\right)-\zeta_{N}^{\prime} \omega\left(\mathbf{k}_{N}^{\prime}\right)-\ldots-\zeta_{N}^{(m)} \omega\left(\mathbf{k}_{N}^{(m)}\right)\right] \frac{\tau_{(N)}}{\varrho}\right\},
\end{aligned}
$$

we obtain the following total oscillatory factor

$$
\exp \left\{\mathrm{i} \frac{1}{\varrho} \Phi{\overrightarrow{\zeta, \vec{\zeta}_{(m), T}}}\left(\mathbf{k}, \vec{k}_{(m)}\right)\right\}
$$

where the phase function $\Phi_{T, \vec{\zeta}}(\vec{k})$ of the monomial is defined by the formula

$$
\Phi_{T, \vec{\zeta}}(\vec{k}, \vec{\tau})=\sum_{N \in T}\left[\zeta_{N} \omega(\mathbf{k})-\sum_{i=1}^{\mu(N)} \zeta_{N}^{\left(c_{i}(N)\right)} \omega\left(\mathbf{k}_{c_{i}(N)}\right)\right] \tau_{(N)}
$$

The vectors $\vec{k}, \vec{\tau}$ and $\vec{\zeta}$ are composed of $\mathbf{k}_{N}, \tau_{N}$ and $\zeta_{N}$ using the standard labeling of the nodes. 
Notice then that the oscillatory exponent (8.1) is the only expression in the composition monomial which involves parameter $\varrho$. Observe also that the FM condition takes here the form

$$
\zeta_{N}=\sum_{i=1}^{\mu(N)} \zeta_{N}^{\left(c_{i}(N)\right)}
$$

The domain of integration with respect to time variables is given in terms of the tree $T$ by the following inequalities

$$
D_{T}=\left\{\tau_{(N)}: 0 \leq \tau_{(N)} \leq \tau_{(p(N))}, N \in T \backslash N_{*}\right\}
$$

where $p(N)$ is the parent node of the node $N$. Using introduced notations we can write the action of the monomial $M\left(\mathcal{F}, T, \vec{\lambda}_{(\hat{s})}, \vec{\zeta}_{(m)}\right)$ in the form

$$
\begin{gathered}
M(\mathcal{F}, T, \vec{\lambda}, \vec{\zeta})\left(\prod_{\operatorname{rank}(N)=0} \tilde{\mathbf{h}}_{N}\right)\left(\mathbf{k}_{N_{*}}, \tau_{N_{*}}\right)=\int_{D_{T}}\left(\prod_{N \neq N_{*}} \int_{[-\pi, \pi]^{\mu(N) d}}\right) \\
\quad \exp \left\{i \frac{1}{\mathrm{i}-\Phi} \Phi_{T, \vec{\zeta}}(\vec{k}, \vec{\tau})\right\} M(\chi, T, \vec{\lambda}, \vec{\zeta}, \vec{k}) \prod_{\operatorname{rank}(N)=0} \tilde{\mathbf{h}}_{N}\left(\mathbf{k}_{N}\right) \\
\prod_{\operatorname{rank}(N)>0} \delta\left(\mathbf{k}_{N}-\mathbf{k}_{c_{1}(N)}-\cdots-\mathbf{k}_{c_{\mu(N)}(N)}\right) \prod_{N \neq N_{*}} \mathrm{~d} \mathbf{k}_{N} \prod_{N \neq N_{*}} \mathrm{~d} \tau_{(N)} .
\end{gathered}
$$

Note that $m$ equals the number of end nodes, that is nodes with zero rank and they are numerated using the standard labeling of the nodes, that is

$$
\tilde{\mathbf{h}}_{1}\left(\mathbf{k}_{1}\right) \cdots \tilde{\mathbf{h}}_{m}\left(\mathbf{k}_{m}\right)=\prod_{\operatorname{rank}(N)=0} \tilde{\mathbf{h}}_{N}\left(\mathbf{k}_{N}\right)
$$

The formula (8.4) gives a closed form of a composition monomial based on oscillatory integral operators $\mathcal{F}^{(m)}$ with an arbitrary large rank.

\section{Appendix B: Proof of the refined implicit function theorem}

Here we give the proof of Theorem 4.25 .

First, we consider the following elementary problem which provides majorants for the problem of interest. Let a function of one complex variable be defined by the formula

$$
\check{\mathcal{F}}(u)=C_{\mathcal{F}} \sum_{m=2}^{\infty} u^{m} R_{\mathcal{F}}^{-m}=C_{\mathcal{F}}\left[\frac{u^{2} / R_{\mathcal{F}}^{2}}{1-u / R_{\mathcal{F}}}\right], C_{\mathcal{F}}>0, R_{\mathcal{F}}>0 .
$$


In this case $\check{\mathcal{F}}^{(m)}\left(x_{1} \ldots x_{m}\right)=C_{\mathcal{F}} R_{\mathcal{F}}^{-m} x_{1} \ldots x_{m}$. Let us introduce the equation

$$
u=\check{\mathcal{F}}(u)+x, u, x \in \mathbb{C}
$$

which is a particular case of (4.1). A small solution $u(x)$ of this equation such that $u(0)=0$ is given by the series

$$
u=\check{G}(x)=\sum_{m=1}^{\infty} \check{G}^{(m)} x^{m},
$$

which is a particular case of formula (4.14). The terms $\check{G}^{(m)} x^{m}$ of this problem are determined from (4.18) and can be written in the form (4.29)

$$
\check{G}^{(m)} x^{m}=\sum_{T \in T_{m}} c_{T} M(\check{\mathcal{F}}, T) x^{m} .
$$

Obviously,

$$
M(\check{\mathcal{F}}, T) x^{m}=C_{\mathcal{F}}^{i(T)} R_{\mathcal{F}}^{-e(T)} x^{m}
$$

where $i(T)$ is the incidence number of the tree $T, e(T)$ is the number of edges of $T$. Now we compare solution of the general equation (4.1). It is given by the formula (4.14) with operators $\mathcal{G}^{(m)}\left(\mathbf{u}^{m}\right)$ admitting expansion (4.29). Since

$$
\left\|\mathcal{F}^{(m)}\right\| \leq C_{\mathcal{F}} R_{\mathcal{F}}^{-m}
$$

where the constants are the same as in (9.1) we have

$$
\left\|M(\mathcal{F}, T)\left(\mathbf{x}_{1} \ldots \mathbf{x}_{\nu}\right)\right\| \leq M(\check{\mathcal{F}}, T)\left\|\mathbf{x}_{1}\right\| \ldots\left\|\mathbf{x}_{\nu}\right\|,
$$

implying

$$
\sum_{T \in T_{m}} c_{T}\left\|M(\mathcal{F}, T)\left(\mathbf{x}_{1} \ldots \mathbf{x}_{m}\right)\right\| \leq \sum_{T \in T_{m}} c_{T} M(\check{\mathcal{F}}, T)\left\|\mathbf{x}_{1}\right\| \ldots\left\|\mathbf{x}_{m}\right\|=\check{G}^{(m)}\left\|\mathbf{x}_{1}\right\| \ldots\left\|\mathbf{x}_{m}\right\| .
$$

Solving (9.2) we get explicitly

$$
u=\frac{R_{\mathcal{F}}}{2 c}\left(1-\sqrt{1-4 c \frac{x}{R_{\mathcal{F}}}}\right)=\check{G}(x), c=\frac{C_{\mathcal{F}}}{R_{\mathcal{F}}}+1 .
$$

We have the following estimate of the coefficients

$$
\check{G}^{(m)} \leq \frac{R_{\mathcal{F}}^{2}}{2\left(C_{\mathcal{F}}+R_{\mathcal{F}}\right)}\left(4 \frac{C_{\mathcal{F}}+R_{\mathcal{F}}}{R_{\mathcal{F}}^{2}}\right)^{m}, m=1,2, \ldots,
$$

(see 4 for details in a similar situation). From (9.4) and (9.6) we infer the following inequality

$$
\sum_{T \in T_{m}} c_{T} C_{\mathcal{F}}^{i(T)} R_{\mathcal{F}}^{-e(T)} \leq \frac{R_{\mathcal{F}}^{2}}{2\left(C_{\mathcal{F}}+R_{\mathcal{F}}\right)}\left(4 \frac{C_{\mathcal{F}}+R_{\mathcal{F}}}{R_{\mathcal{F}}^{2}}\right)^{m}
$$

which hods for all $C_{\mathcal{F}}, R_{\mathcal{F}}>0$. We set $C_{\mathcal{F}}=R_{\mathcal{F}}=1$ and obtain the desired bound (4.35). 


\section{Notations and abbreviations}

For reader's convenience we provide below a list of notations and abbreviations used in this paper.

AFM- alternatively frequency matched, see Definition 5.19

ANFM - alternatively non-frequency-matched see Definition 5.19]

band-crossing points - see Definition 2.3

cc - complex conjugate to the preceding terms in the formula

composition monomial - see Definition 4.9

decoration projections - see (4.36), (4.37)

decorated monomial - see Definition 4.20

CI monomials - cross-interacting monomials, see Definition 5.8

FPU, Fermi-Pasta-Ulam equation - (2.10), (2.11), (17.1)

Floquet-Bloch modal decomposition - see (17.14)

Fourier transform - see (2.59)

FM - frequency matched, see Definition [5.10 see also (5.42)

homogeneity index of a monomial Definition 4.9]

homogeneity index of a tree - Definition 4.11

incidence number of a monomial - number of occurrences of operators $\mathcal{F}^{(l)}$ in the composition monomial

incidence number of a monomial - see Definition 4.10

incidence number of a tree - Definition 4.12

lattice Fourier transform - see (2.2)

monomial - Definition 4.9

NFM - non-frequency-matched see Definition [5.10, see also (5.46)

oscillatory integral operator - see (3.8), (3.3)

rank of monomial - see Definition 4.9

root operator (4.20)

SI monomials - self-interacting monomials, see Definition 5.8

Schwartz functions - infinitely smooth functions on $\mathbb{R}^{d}$ which decay faster than any power, see (7.6)

single-mode wavepacket - see Definition 2.9

submonomial (4.10)

wavepacket see Definition 2.9

$\tilde{\mathrm{d}}^{(m-1)} \vec{k}=\frac{1}{(2 \pi)^{(m-1) d}} \mathrm{~d} \mathbf{k}^{\prime} \ldots \mathrm{d} \mathbf{k}^{(m-1)}-$ see (2.24)

$\mathbb{D}_{m}=[-\pi, \pi]^{(m-1) d}-$ see $(2.23)$ or $\mathbb{D}_{m}=\mathbb{R}^{(m-1) d}$ see (2.65)

$E=C\left(\left[0, \tau_{*}\right], L_{1}\right)$ see (2.30)

$\hat{F}^{(m)}-m$-linear operator in $L_{1}$, see (2.22), (2.64)

$\mathcal{F}_{n, \zeta, \vec{n}, \vec{\zeta}}^{(m)}$ - basis element of the $m$-linear operator $\mathcal{F}^{(m)}$ in $E$ see (3.8)

$\mathcal{F}_{\lambda, \vec{\zeta}}^{(n)}-$ see (4.43) 
$\hat{h}_{\zeta}(\beta, \mathbf{k}), \zeta= \pm-$ Fourier transform of the wavepacket initial data $h_{\zeta}(\beta, \mathbf{r})$, see Definition 2.9

$\hat{h}_{\zeta}\left(\frac{1}{\beta} \boldsymbol{\xi}\right), \zeta= \pm-$ Fourier transform of the wavepacket $h_{\zeta}(\beta \mathbf{r})$ initial data, see Definition 2.9

$\tilde{\mathbf{h}}_{l}^{\Psi}(\mathbf{k}, \beta)$ - a function nullified outside $\beta^{1-\epsilon}$ vicinity of $\pm \mathbf{k}_{*}$, see (5.15)

$\mathbf{k}=\left(k_{1}, \ldots, k_{d}\right) \in[-\pi, \pi]^{d}$ - quasimomentum (wave vector) variable, (2.2), (2.25).

$\mathbf{k}=\left(k_{1}, \ldots, k_{d}\right) \in \mathbb{R}^{d}-$ Fourier wave vector variable, (2.59), (2.25).

$\mathbf{k}_{*}=\left(k_{* 1}, \ldots, k_{* d}\right)-$ center of the wavepacket see Definition 2.9]

$\mathbf{k}_{* l}$ - center of $l$-th wavepacket

$\vec{k}=\left(\mathbf{k}^{\prime}, \ldots, \mathbf{k}^{(m)}\right)$, - interaction multi-wave vector, (2.25), (3.7) .

$\mathbf{k}^{(s)}(\mathbf{k}, \vec{k})=\mathbf{k}-\mathbf{k}^{\prime}-\ldots-\mathbf{k}^{(s-1)}-$ see (2.25)

$L_{1}$ - Lebesgue space $L_{1}\left([-\pi, \pi]^{d}\right)$ or $L_{1}\left(\mathbb{R}^{d}\right)$ - see (2.31) and (2.66)

$n$ - band number

$\vec{n}=\left(n^{\prime}, \ldots, n^{(m)}\right)-$ band interaction index, (3.7)

$\nabla_{\mathbf{r}}=\left(\frac{\partial}{\partial r_{1}}, \frac{\partial}{\partial r_{2}}, \cdots, \frac{\partial}{\partial r_{d}}\right)-$ spatial gradient

$O(\mu)$ - any quantity having the property that $\frac{O(\mu)}{\mu}$ is bounded as $\mu \rightarrow 0$.

$\omega_{\bar{n}}(\mathbf{k})=\zeta \omega_{n}(\mathbf{k})$ - dispersion relation of the band $(\zeta, n)$, see (2.13)

$\omega_{n_{0}}^{\prime}(\mathbf{k})=\nabla_{\mathbf{k}} \omega_{n_{0}}(\mathbf{k})-$ group velocity vector

$\omega_{n}(\mathbf{k})-n$-th eigenvalue of $\mathbf{L}(\mathbf{k})$, see (2.13); dispersion relation of $n$-th band

$\Psi$ - cutoff function in quasimomentum domain, see (5.12)

$\phi_{\vec{n}}(\mathbf{k}, \vec{k})=\zeta \omega_{n}(\mathbf{k})-\zeta^{\prime} \omega_{n^{\prime}}\left(\mathbf{k}^{\prime}\right)-\ldots-\zeta^{(m)} \omega_{n^{(m)}}\left(\mathbf{k}^{(m)}\right)-$ interaction phase function, (3.9)

$\pi_{0}-$ see (5.13)

$\Pi_{n, \zeta}(\mathbf{k})$ - projection in $\mathbb{C}^{2 J}$ onto direction of $\mathbf{g}_{n, \zeta}(\mathbf{k})$; see (2.19)

$\mathbf{r}=\left(r_{1}, \ldots, r_{d}\right)-$ spatial variable

$\varrho=\beta^{2}-(2.46)$

$\sigma$ - the set of band-crossing points, see Definition 2.3

$\hat{\mathbf{U}}(\mathbf{k})$ - Fourier transform of $U(\mathbf{r})$, see (2.59)

$\tilde{\mathbf{U}}_{n, \zeta}(\mathbf{k}, \tau)=\tilde{\mathbf{u}}_{n, \zeta}(\mathbf{k}, \tau) \mathrm{e}^{-\frac{\mathrm{i} \tau}{\varrho} \zeta \omega_{n}(\mathbf{k})}-$ amplitudes, see (3.2)

$\zeta= \pm$ or $\zeta= \pm 1$ - band binary index.

$\vec{\zeta}=\left(\zeta^{\prime}, \ldots, \zeta^{(m)}\right)$ - binary band index vector, see (3.7)

$Z^{*}$ - complex conjugate to $Z$

Acknowledgment: Effort of A. Babin and A. Figotin is sponsored by the Air Force Office of Scientific Research, Air Force Materials Command, USAF, under grant number FA9550-04-1-0359. 


\section{References}

[1] Babin A. and Figotin A., Nonlinear Photonic Crystals: I. Quadratic nonlinearity, Waves in Random Media, 11, R31-R102, (2001).

[2] Babin A. and Figotin A., Nonlinear Photonic Crystals: II. Interaction classification for quadratic nonlinearities, Waves in Random Media, 12, R25-R52, (2002).

[3] Babin A. and Figotin A., Nonlinear Photonic Crystals: III. Cubic Nonlinearity, Waves in Random Media, 13, pp. R41-R69 (2003).

[4] Babin A. and Figotin A., Nonlinear Maxwell Equations in Inhomogenious Media, Commun. Math. Phys. 241, 519-581 (2003).

[5] Babin A. and Figotin A., Polylinear spectral decomposition for nonlinear Maxwell equations, in Partial Differential Equations, M.S. Agranovich and M.A. Shubin eds, Advances in Mathematical Sciences, American Mathematical Society Translations -Series 2, Vol. 206, 2002, p. 1-28.

[6] Babin A. and Figotin A., Nonlinear Photonic Crystals: IV Nonlinear Schrodinger Equation Regime, Waves in Random and Complex Media, Vol. 15, No. 2 (2005), pp. 145-228.

[7] Babin A. and Figotin A., Wavepacket preservation under nonlinear evolution, submitted; e-print available online at arxiv.org arXiv:math.AP/0607723

[8] Babin A., Mahalov A. and Nicolaenko B., Global regularity of 3D rotating Navier-Stokes equations for resonant domains, Indiana University Mathematics Journal vol. 48 no. 3 (1999), p.1133-1176.

[9] Babin A., Mahalov A. and Nicolaenko B, Fast Singular Oscillating Limits and Global Regularity for the 3D Primitive Equations of Geophysics, M2AN,v.34,no.2, 2000, p.201222 .

[10] Bambusi, D., Birkhoff normal form for some nonlinear PDEs, Comm. Math. Phys. 234 (2003), no. 2, 253-285.

[11] Ben Youssef, W.; Lannes, D., The long wave limit for a general class of 2D quasilinear hyperbolic problems, Comm. Partial Differential Equations 27 (2002), no. 5-6, 979-1020.

[12] Berman G.P, Izrailev F.M., The Fermi-Pasta-Ulam problem: 50 years of progress, arXiv:nlin.CD

[13] Bogoliubov N. N. and Mitropolsky Y. A., Asymptotic Methods In The Theory Of NonLinear Oscillations, Delhi, Hindustan Pub. Corp., 1961.

[14] Bona, J. L.; Colin, T.; Lannes, D., Long wave approximations for water waves, Arch. Ration. Mech. Anal. 178 (2005), no. 3, 373-410. 
[15] Bourgain, J., Global solutions of nonlinear Schrödinger equations. American Mathematical Society Colloquium Publications, 46. American Mathematical Society, Providence, RI, 1999.

[16] Cazenave T., Semilinear Schrödinger equations. Courant Lecture Notes in Mathematics, 10., New York University, Courant Institute of Mathematical Sciences, New York; American Mathematical Society, Providence, RI, 2003.

[17] Colin, T., Rigorous derivation of the nonlinear Schrödinger equation and DaveyStewartson systems from quadratic hyperbolic systems, Asymptot. Anal. 31 (2002), no. 1, 69-91.

[18] Colin, T.; Lannes, D., Justification of and long-wave correction to Davey-Stewartson systems from quadratic hyperbolic systems., Discrete Contin. Dyn. Syst. 11 (2004), no. $1,83-100$.

[19] Craig W.; Groves M. D., Normal forms for wave motion in fluid interfaces, Wave Motion 31 (2000), no. 1, 21-41.

[20] Craig, W.; Sulem, C.; Sulem, P.-L., Nonlinear modulation of gravity waves: a rigorous approach, Nonlinearity 5 (1992), no. 2, 497-522.

[21] Dineen S., Complex Analysis on Infinite Dimensional Spaces, Springer, 1999.

[22] Gallay T.; Wayne C. E., Invariant manifolds and the long-time asymptotics of the NavierStokes and vorticity equations on $\mathbf{R}^{2}$., Arch. Ration. Mech. Anal. 163 (2002), no. 3, 209-258.

[23] Giannoulis, J.; Mielke, A., The nonlinear Schrödinger equation as a macroscopic limit for an oscillator chain with cubic nonlinearities., Nonlinearity 17 (2004), no. 2, 551-565.

[24] N. Hayashi and P. Naumkin, Asymptotics of small solutions to nonlinear Schrödinger equations with cubic nonlinearities. Int. J. Pure Appl. Math. 3 (2002), no. 3, 255-273.

[25] Hille E. and Phillips R. S., Functional Analysis and Semigroups, AMS, 1991.

[26] Infeld, E. and Rowlands, G. Nonlinear Waves, Solitons, and Chaos, 2nd ed. Cambridge, England: Cambridge University Press, 2000.

[27] Iooss, G.; Lombardi, E., Polynomial normal forms with exponentially small remainder for analytic vector fields. J. Differential Equations 212 (2005), no. 1, 1-61.

[28] Joly, J.-L.; Metivier, G.; Rauch, J., Diffractive nonlinear geometric optics with rectification, Indiana Univ. Math. J. 47 (1998), no. 4, 1167-1241.

[29] Kalyakin, L. A., Long-wave asymptotics. Integrable equations as the asymptotic limit of nonlinear systems., Uspekhi Mat. Nauk 44 (1989), no. 1(265), 5-34, 247; translation in Russian Math. Surveys 44 (1989), no. 1, 3-42. 
[30] Kalyakin L.A., Asymptotic decay of a one-dimensional wave packet in a nonlinear dispersive medium, Math. USSR Sb. Surveys 60 (2) (1988) 457-483.

[31] Kuksin S. B., Fifteen years of KAM for PDE. Geometry, topology, and mathematical physics, 237-258, Amer. Math. Soc. Transl. Ser. 2, 212, Amer. Math. Soc., Providence, RI, 2004.

[32] Kirrmann P.; Schneider G.; Mielke A., The validity of modulation equations for extended systems with cubic nonlinearities, Proc. Roy. Soc. Edinburgh Sect. A 122 (1992), no. $1-2,85-91$.

[33] Lax P.D., Integrals of nonlinear equations of evolution and solitary waves, Comm. Pure Appl. Math. 21 (1968), 467-490.

[34] Maslov V.P., Non-standard characteristics in asymptotic problems, Uspekhi Mat. Nauk 38:6 (1983), 3-36, translation in Russian Math. Surveys 38:6 (1983),1-42.

[35] Mielke A., Schneider G., Ziegra A., Comparison of inertial manifolds and application to modulated systems, Math. Nachr. 214 (2000), 53-69.

[36] Nayfeh, A. H., Perturbation Methods, New York: Wiley, 1973.

[37] A. Pankov, Travelling Waves And Periodic Oscillations In Fermi-Pasta-Ulam Lattices, Imperial College Press, 2005.

[38] Pierce R. D.; Wayne C. E., On the validity of mean-field amplitude equations for counterpropagating wavetrains, Nonlinearity 8 (1995), no. 5, 769-779.

[39] Schneider, G., Justification of modulation equations for hyperbolic systems via normal forms, NoDEA Nonlinear Differential Equations Appl. 5 (1998), no. 1, 69-82.

[40] Schneider, G., Justification and failure of the nonlinear Schrödinger equation in case of non-trivial quadratic resonances. J. Differential Equations 216 (2005), no. 2, 354-386.

[41] Schneider G., Uecker H. Existence and stability of modulating pulse solutions in Maxwell's equations describing nonlinear optics, Z. Angew. Math. Phys. 54 (2003), no. 4, 677-712.

[42] Sulem C. and Sulem P.-L. , The Nonlinear Schrodinger Equation, Springer , 1999.

[43] Soffer A., Weinstein M. I., Resonances, radiation damping and instability in Hamiltonian nonlinear wave equations, Invent. Math. 136 (1999), no. 1, 9-74.

[44] Weissert T.P.,The Genesis of Simulation in Dynamics: pursuing the Fermi-Pasta-Ulam problem, Springer-Verlag, New York, 1997.

[45] Whitham G., Linear and Nonlinear Waves, John Wiley \& Sons, 1974. 